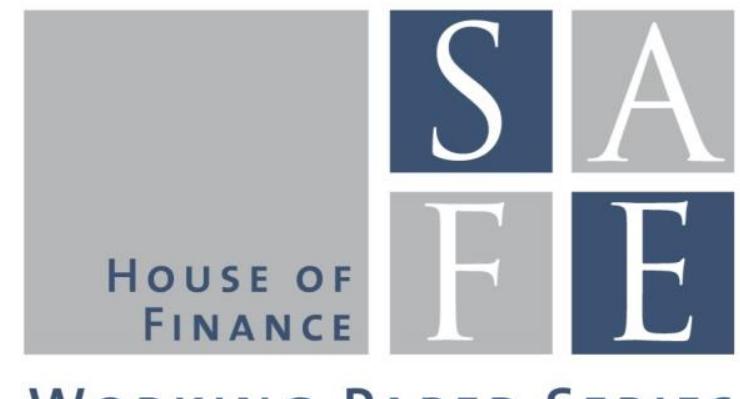

WORKINg PAPER SERIES

Holger Kraft - Claus Munk - Sebastian Wagner

\title{
Housing Habits and their Implications for Life-Cycle Consumption and Investment
}

SAFE Working Paper No. 85

SAFE I Sustainable Architecture for Finance in Europe A cooperation of the Center for Financial Studies and Goethe University Frankfurt 


\section{Non-Technical Summary}

Empirical studies find that perishable consumption is hump shaped over the life cycle, being lower at the start and at the end. However, standard frictionless consumptionsavings models with one consumption good suggest that optimal consumption should be either increasing, decreasing, or flat over life. Additionally, these models generate optimal stock demands that are unrealistically high. Finally, most studies do not involve housing decisions. Those including housing however usually predict optimal housing expenditures that are neither age nor wealth dependent, which is not in line with empirical observations.

In order to find a theoretical explanation for these stylized facts, we set up a life-cycle model of household decisions involving consumption of both perishable goods and housing services, labor income, house prices, home renting and owning, stock investments, and portfolio constraints. The model features habit formation for housing consumption, which leads to optimal decisions closer in line with empirical observations. We start with a model with full certainty and use the closed-form solutions to build intuition for the economic forces at play. After a model with spanned risk and no frictions the full model features portfolio constraints and unspanned income risk, which we solve using an innovative numerical solution method for problems with a high number of state variables. In addition to these models we provide extensions to mortality risk and robustness checks for different income profiles based on education. Our input data for stock prices, house prices, labor income and correlations are estimated on US data between 1953 and 2010.

Our results show that the model can generate various stylized facts that seem puzzling through the lens of standard life-cycle models. First, stock investments are low or zero for many young agents and then gradually increase over life. Housing investments crowd out stock investments, in particular for young agents, because housing investments (i) provide a hedge against increases in the price of housing consumption, (ii) ensure that the agent can obtain the future minimum housing consumption generated by her habits, and (iii) are not a bad investment from a purely speculative view. Secondly, our model generates an age- and wealth-dependent housing expenditure share instead of the counter-factually constant share found in standard models. Thirdly, in our model perishable consumption is much more sensitive to wealth and income shocks than housing consumption, in particular for young agents. Finally, the housing habit helps in explaining the humpshaped life-cycle pattern in non-housing consumption. 


\title{
Housing Habits and their Implications for Life-Cycle Consumption and Investment
}

\author{
Holger Kraft $^{\mathrm{a}} \quad$ Claus Munk $^{\mathrm{b}} \quad$ Sebastian Wagner $^{\mathrm{c}}$
}

February 18, 2015

\begin{abstract}
We set up and solve a rich life-cycle model of household decisions involving consumption of both perishable goods and housing services, stochastic and unspanned labor income, stochastic house prices, home renting and owning, stock investments, and portfolio constraints. The model features habit formation for housing consumption, which leads to optimal decisions closer in line with empirical observations. Our model can explain (i) that stock investments are low or zero for many young agents and then gradually increasing over life, (ii) that the housing expenditure share is age- and wealthdependent, (iii) that perishable consumption is more sensitive to wealth and income shocks than housing consumption, and (iv) that non-housing consumption is hump-shaped over life.
\end{abstract}

Keywords: Habit formation, life-cycle household decisions, housing expenditure share, consumption hump, stock market participation, renting vs. owning home, human capital

JEL subject codes: G10, D14, D91, E21, R21

\footnotetext{
${ }^{a}$ Department of Finance, Goethe University Frankfurt am Main, Faculty of Economics and Business Administration, Germany. E-mail: holgerkraft@finance.uni-frankfurt.de

b Department of Finance, Copenhagen Business School, Denmark. E-mail: cm.fi@cbs.dk

${ }^{c}$ Department of Finance, Goethe University Frankfurt am Main, Faculty of Economics and Business Administration, Germany. E-mail: Sebastian.Wagner@hof.uni-frankfurt.de

We appreciate comments received at presentations at the University of York and the Copenhagen Business School. We are grateful to Fang Yang for sharing her data with us. Holger Kraft gratefully acknowledges research and financial support from the Center of Excellence SAFE, funded by the State of Hessen research initiative LOEWE, and both Holger Kraft and Sebastian Wagner gratefully acknowledge financial support by Deutsche Forschungsgemeinschaft (DFG).
} 


\section{Contents}

1 Introduction 1

$\begin{array}{llr}2 & \text { Related literature } & 6\end{array}$

3 Model ingredients $\quad 8$

3.1 Consumption goods . . . . . . . . . . . . . . . . 8

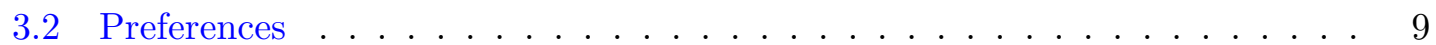

3.3 Labor income . . . . . . . . . . . . . . . . . . . . . . . . . 10

3.4 Investments, wealth, and potential constraints . . . . . . . . . . . 10

4 Two instructive models with closed-form solutions 11

4.1 Model with full certainty . . . . . . . . . . . . . . . . . . . . . 11

4.2 A model with spanned risks and no frictions . . . . . . . . . . . . . . . 15

5 The full-fledged model $\quad 19$

5.1 Model specification . . . . . . . . . . . . . . . . . . . . . . . . . . 19

5.2 Outline of the solution approach . . . . . . . . . . . . 20

5.3 A family of artificial markets . . . . . . . . . . . . . . . . . . 21

5.4 Optimal decisions in the artificial markets . . . . . . . . . . . . . 23

5.5 Consumption and investment decisions in the true market . . . . . . . . 25

6 Results with benchmark parameter values $\quad 27$

7 Some model extensions and robustness checks 32

8 Conclusion $\quad 35$

$\begin{array}{ll}\text { A Proofs } & 37\end{array}$

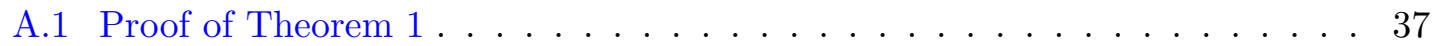

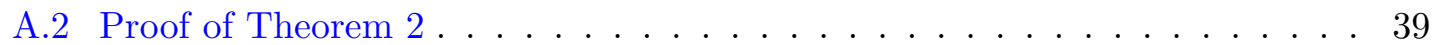

A.3 Proof of Lemma $1 \ldots \ldots \ldots$. . . . . . . . . . . . . . . . . . 43

A.4 Proof of Theorem 3 . . . . . . . . . . . . . . . . . . 44

B Extensions to bequest and mortality risk $\quad 49$

B.1 The model of Section 4.1 . . . . . . . . . . . . . . . . . . . 50

B.2 The model of Section $4.2 \ldots \ldots \ldots \ldots \ldots \ldots$

B.3 The full-fledged model of Section $5 \ldots \ldots \ldots \ldots$

$\begin{array}{ll}\text { References } & 56\end{array}$ 


\section{Introduction}

Residential real estate is important both as a consumption good and an investment which is illustrated by the following key figures. Housing services (shelter) has a weight of $31.9 \%$ in the November 2013 U.S. Consumer Price Index for All Urban Consumers. In 2010 the value of residential property owned by U.S. households was $36 \%$ of total household wealth with an even higher share for middle-income households. The home ownership rate has been in the range 65-70\% in the U.S. in the period 1996-2013 and is also well above $50 \%$ in most other developed countries. Moreover, 27\% of U.S. home sales in 2011 were for investment purposes. ${ }^{1}$ In addition, used as collateral, real estate facilitates household borrowing. For these reasons housing consumption and investment should be included in life-cycle models of optimal household decisions but, because of the significant increase in modeling complexity, only few existing papers do so.

We solve for the optimal life-cycle decisions in a rich model featuring consumption of both perishable goods and housing services, stochastic and unspanned labor income, stochastic house prices, renting/owning decisions, stock investments, and portfolio constraints. A distinct and innovative feature of our model is habit formation in the preferences for housing consumption. ${ }^{2}$ Habit formation in preferences finds empirical support, and the consequences of habits in perishable consumption for both consumption and investment decisions are well-studied (Section 2 reviews the literature). In contrast, the impact of habits in consumption of durable goods and, in particular, housing remains unexplored, even though habit formation seems even more relevant for housing consumption than for perishable consumption. The residence of an agent serves as a very visible status symbol, and the individual might build up affection for the home and the neighborhood as well as social ties to other persons in the community. ${ }^{3}$ Moreover, explicit and implicit costs of home transactions are likely to induce consumption and trading patterns similar to those caused by habit persistence. For these reasons the agent might be very reluctant to reduce housing consumption relative to other goods in dire times.

Our model explains key features of observed consumption and investment choices:

\footnotetext{
${ }^{1}$ The Consumer Price Index is described by the Bureau of Labor Statistics at http://www.bls.gov/ $\mathrm{cpi} /$. The wealth share of real estate is from the Survey of Consumer Finances, see Tables 8 and 9.1 in Bricker, Kennickell, Moore, and Sabelhaus (2012); residential property includes primary residence and other residential property but not equity in non-residential property. Similar numbers are reported by Campbell (2006, Figures 2 and 3) and Guiso and Sodini (2013). For home ownership rates, see the Census Bureau at http://www.census.gov/housing/hvs/ for the U.S. and Andrews and Sánchez (2011) for selected OECD countries. The pure investment share of U.S. home sales is from the Investment and Vacation Home Buyers Survey by the National Association of Realtors, cf. Choi, Hong, Kubik, and Thompson (2013).

${ }^{2}$ We use an internal habit, i.e., the agent's utility of current consumption decreases in her past consumption. An external habit (like "keeping up with the Jones'es") means that the agent compares consumption to an external benchmark (e.g., aggregate consumption) unaffected by her own consumption decisions.

${ }^{3}$ Solnick and Hemenway (2005) report empirical evidence supporting status concerns regarding housing.
} 
Stock investments. In our benchmark parametrization the optimal stock investment is zero in the early years and then gradually increasing until retirement. Typical models including income, but ignoring housing, imply that all financial wealth should be invested in the stock market when young and then the stock weight eventually goes below $100 \%$ and decreases until retirement. Empirically, many individuals invest very little or nothing at all in stocks when young, and the stock market participation seems to be a hump-shaped function of age. Adding housing as a second investment asset reduces the speculative stock demand because of diversification. ${ }^{4}$ Due to the housing habit, the investor maintains a wealth buffer invested in the housing market to finance the future minimum housing consumption implied by the current habit level. Hence, the habit reduces the wealth disposable for speculative investments and thus further reduces the stock investment. The wealth buffer might increase early in life due to a rapidly increasing housing habit level, but eventually as the remaining lifetime shrinks the necessary wealth buffer falls so that the disposable wealth and the speculative stock investment tend to increase. The habitinduced wealth buffer also implies that the fraction of wealth invested in the stock is increasing in wealth as seen in the data.

Perishable consumption. Empirical studies find that perishable consumption is hump shaped over the life cycle, but standard frictionless consumption-savings models with one consumption good lead to optimal consumption being either increasing, decreasing, or flat over life. The consensus in the literature is that the hump is caused by borrowing constraints or non-hedgeable risks, but we show that a hump in perishable consumption naturally emerges in absence of such frictions if the individual forms housing habits. Intuitively, housing consumption early in life is expensive since the individual then commits to a certain level of housing consumption in the remaining lifetime, which requires keeping the above-mentioned wealth buffer financing that minimum consumption. As the required buffer decreases, housing consumption effectively becomes less expensive. Early in life the individual thus tilts the consumption bundle towards perishable consumption, and less so as time passes. By embedding this mechanism in a setting in which overall consumption tends to increase over life, a hump-shaped perishable consumption emerges. With mortality risk, the subjective time preference rate is effectively increasing over life, which can lead to a hump-shaped consumption pattern even in the absence of habit formation, but habit formation leads to an earlier hump in line with the data.

Housing expenditure share. The Cobb-Douglas utility assumed in standard twogood models imply a constant relation between housing consumption expenditures and perishable consumption expenditures, and thus a constant housing expenditure share.

\footnotetext{
${ }^{4}$ This requires that the Sharpe ratio of a housing investment exceeds the product of the Sharpe ratio of the stock index and the house-stock correlation.
} 
This contradicts empirical observations along several dimensions. First, in the data the housing expenditure share varies counter cyclically. For example, the housing expenditure share has a sizeable negative correlation with GDP growth. Figure 1 shows the 1980-2013 annual GDP growth rates in the U.S. together with the annual percentage changes in the housing expenditure share of U.S. households reported in the National Income and Product Accounts (NIPA). The correlation over the full period shown is -0.51 , and in the period 1990-2013 it is even $-0.59 .^{5}$ Between 1980 and 2013 the housing expenditure ratio has varied between $21.0 \%$ (in 2012) and $23.1 \%$ (in 1986) with an average of $22.0 \%$. The variation in housing expenditure shares is confirmed by the Consumer Expenditure Survey (CEX). For the period 1993-2013 the housing expenditure share for all age groups was $32.9 \%$ on average and varied between $31.4 \%$ (in 1993) and 34.4\% (in 2009 and 2010). The correlation between GDP growth and the CEX average housing expenditure share is also negative, although of a smaller magnitude than found using NIPA data. ${ }^{6}$ Furthermore, CEX data show that across households the housing expenditure share is decreasing in income. For example, in the 2013 survey the housing expenditure share in the five income quintiles (from lowest to highest) is $40.0 \%, 36.8 \%, 34.8 \%, 32.4 \%$, and $31.1 \%$, respectively. On a micro level, Chetty and Szeidl (2007) report from Panel Study of Income Dynamics (PSID) data that in the year following a job loss, home owners on average reduce food consumption by $9.1 \%$, but housing consumption by only $0.1 \%$. These observations are explained by habit formation: in times of declining [increasing] wealth or income, housing expenditures are reduced [increased] less than non-housing expenditures.

Secondly, the housing expenditure share varies with age. In a detailed analysis of consumption data from CEX and housing ownership data from the Survey of Consumer Finances (SCF), Yang (2009) finds that, for both renters and owners, non-housing consumption is hump shaped, whereas housing consumption per adult-equivalent increases throughout life, quite steeply early in life and then flattening out. Based on her data, Figure 2 shows the housing expenditure share over the life cycle for renters and owners as well as the average of the two. ${ }^{7}$ Except for short flat parts, the housing expenditure share

\footnotetext{
${ }^{5}$ See NIPA Table 2.3.5 published by the Bureau of Economic Analysis, the U.S. Department of Commerce, at http://www.bea.gov/itable/. Following Piazzesi, Schneider, and Tuzel (2007), the housing expenditure share is the ratio of housing consumption expenditures to the sum of housing and non-housing consumption expenditures. Housing consumption expenditures are represented by the item 'housing and utilities' and non-housing consumption is the sum of 'nondurable goods' (less 'clothing and footwear') and 'services' (less 'housing and utilities'). While NIPA includes a quantity index for housing consumption, Piazzesi et al. (2007) argue that this measure is imprecise so, following their lead, we do not use it.

${ }^{6}$ The CEX data was downloaded from the Bureau of Labor Statistics, the U.S. Department of Labor, at http://www.bls.gov/cex/csxshare.htm. We use the expenditure share for the item 'housing'. We obtain very similar results by using 'shelter' that subtracts various expenditures from 'housing.'

${ }^{7}$ We are grateful to Fang Yang for sharing the data with us. The data includes the housing stock of owners, and we assume that housing consumption is a constant fraction of the stock. The fraction is set to $8.22 \%$ since then 20 -year old owners have the same expenditure share as 20 -year old renters, but the
} 


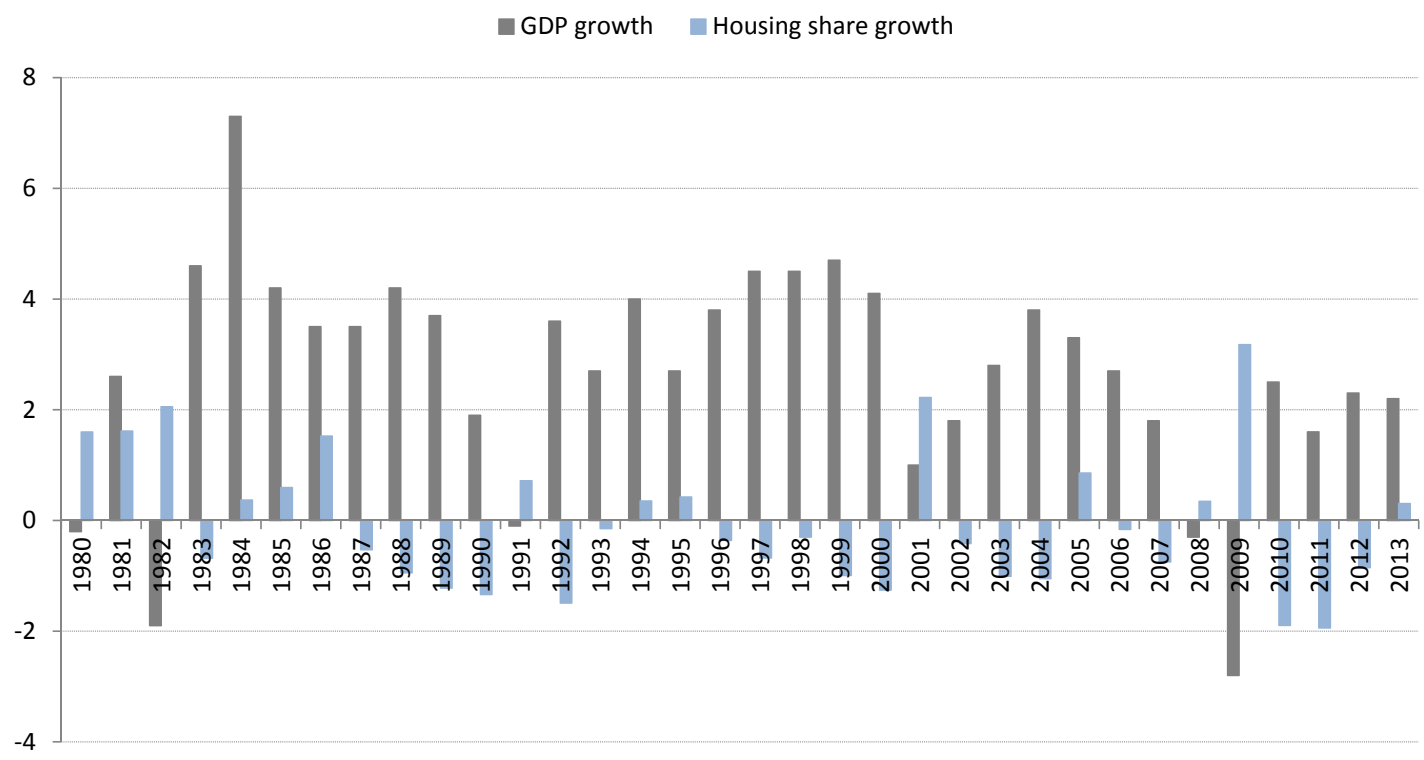

Figure 1: Growth rates of GDP and the housing expenditure share. The data is taken from the U.S. National Income and Product Accounts published by the Bureau of Economic Analysis. GDP growth rates are in Table 1.1.1 (dated January 30, 2015), and the housing expenditure share is in Table 2.3.5 (dated October 30, 2014).

increases with age from around $22 \%$ to $40 \%$, with the steepest increases at age 25-35 and age 60-70. Furthermore, the correlation between the housing expenditure share and GDP growth is more negative for young households than for old. For the 25-34 year olds the correlation is -0.22 , whereas for households above 75 years the correlation is -0.03 . Also these features are consistent with habit formation. Current housing consumption decisions are influenced by their impact on the housing habit in the remaining life, which obviously is less important for households with a shorter expected remaining lifetime.

Housing consumption vs. investment. The optimal housing investment consists of a speculative position, a hedge against increases in future housing consumption costs, a buffer to meet the future costs implied by the current housing habit, and a term adjusting for the extent to which human capital replaces a housing investment. In the benchmark parametrization of our model, the resulting housing investment position is positive from the beginning, then rapidly increasing to around retirement, after which it drops as wealth is reduced to finance retirement consumption. Both in the early and the late years of adult life, the optimal housing consumption exceeds the optimal housing investment, which can be implemented by renting the home and having a limited investment in the housing market, maybe through REITs (Real Estate Investment Trusts). In the intermediate life

life-cycle profile of the expenditure share is the same for a wide range of values for this constant. We use the mean housing and non-housing consumption for each age to compute the expenditure share. 


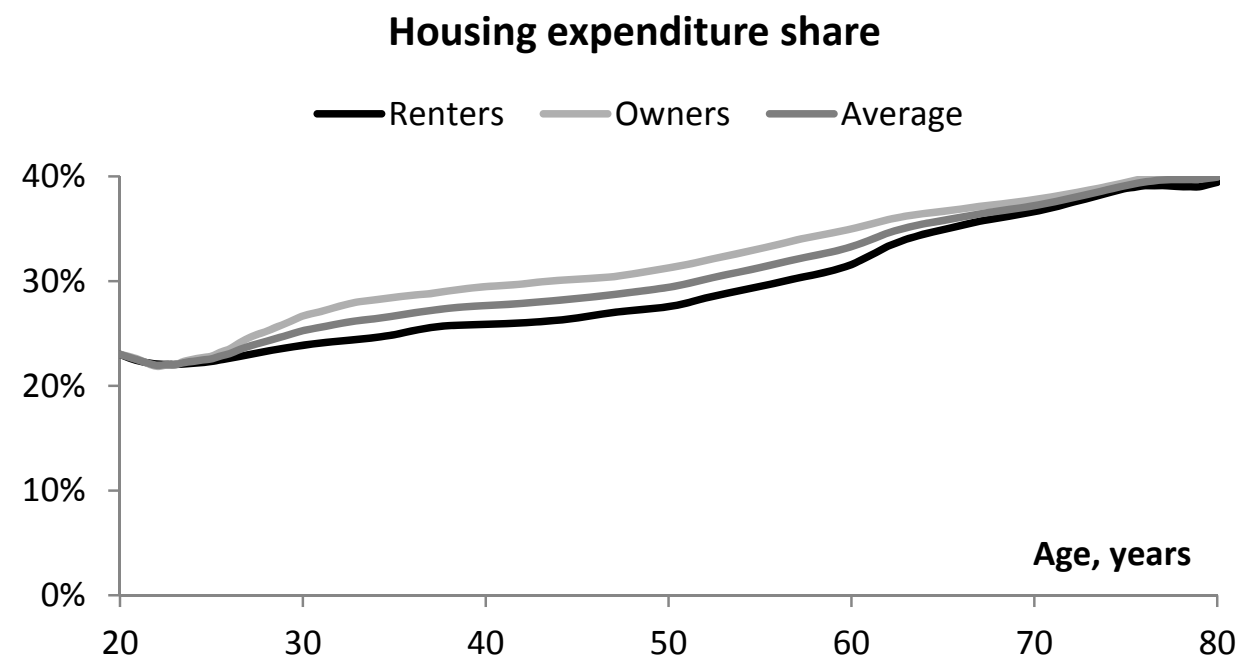

Figure 2: The housing expenditure share over the life cycle. The figure shows housing expenditure shares of renters and owners, as well as the average of the two, over the life cycle. The housing expenditure share is the ratio of housing consumption to the sum of housing and non-housing consumption. The data is based on CEX and SCF, and processed and supplied by Yang (2009).

phase, the optimal housing investment exceeds consumption, which can be implemented by owning the home and investing additionally in the housing market. This renting-owningrenting life-cycle pattern seems consistent with typical real-life behavior.

Our strategy to illustrate the above results is the following. As our full model features portfolio constraints and unspanned labor income risk, the optimal consumption and investment decisions are determined by a numerical method. To better understand the economic forces at play, we first solve two simpler versions of the model in closed form. The first model is designed to demonstrate the impact of the housing habit on the optimal perishable and housing consumption. The second model focuses on the impact of housing and the housing habit on portfolio decisions, but has to abstract from portfolio constraints and unspanned income risk to facilitate a closed-form solution.

To solve the full model, standard grid-based dynamic programming techniques are computationally infeasible due to the high number of state variables (time, wealth, income, house price, habit level). Instead, we extend and adapt the SAMS (Simulation of Artificial Market Strategies) method introduced by Bick, Kraft, and Munk (2013) to habit formation, two consumption goods, and two risky assets. The method optimizes over a family of consumption and investment strategies parameterized by a low number of constants. Each strategy is a minor transformation of the optimal strategy in a closely related unconstrained, complete market, and we derive this optimal strategy in closed form. The expected utility in the true market of each strategy is evaluated by Monte Carlo simula- 
tions and, by embedding that evaluation in a standard numerical optimization over the parameters, we determine the best of these strategies. We show that this strategy deviates from the unknown, truly optimal strategy by at most a few percent in terms of certainty equivalent of wealth, whereas standard numerical methods offer no similar measure of precision.

The rest of the paper is organized as follows. Section 2 positions our paper relative to the existing literature. Section 3 introduces the ingredients of our model. The two simple models are solved in Section 4. The full model and the numerical solution technique are presented in Section 5. Results for a benchmark parametrization of the model are discussed in Section 6. Section 7 considers alternative parametrizations and extensions of the model. Section 8 summarizes and concludes.

\section{Related literature}

The theoretical literature on life-cycle consumption and investments builds on Samuelson (1969) and Merton $(1969,1971)$. The effects of including realistic labor income dynamics and associated portfolio constraints are now well-explored, cf., e.g., Bodie, Merton, and Samuelson (1992), Heaton and Lucas (1997), Cocco, Gomes, and Maenhout (2005), Koijen, Nijman, and Werker (2010), Munk and Sørensen (2010), and Lynch and Tan (2011). Unless the labor income is highly correlated with the stock - or the two processes are co-integrated as assumed by Benzoni, Collin-Dufresne, and Goldstein (2007) - an agent facing borrowing constraints optimally invests $100 \%$ of financial wealth early in life and eventually reduces the weight when approaching retirement. This is at odds with the low stock market participation and the life-cycle variations in the stock weight observed empirically (see, e.g., Guiso and Sodini 2013).

Cocco (2005) adds housing to the setting, but ignores the renting/owning decision and assumes a perfect correlation between house prices and aggregate income shocks. He concludes that house price risk crowds out stock holdings which, in combination with a sizable one-time stock market entry cost, can explain the limited stock market participation. We arrive at the same conclusion in a more general model without imposing a fixed entry cost. Yao and Zhang (2005) generalize Cocco's analysis to an imperfect house-income correlation and endogenize the renting/owning decision, but so that a renter has zero wealth exposure to house price risk and a home owner must have a housing consumption identical to the housing investment position. They find that home owners invest less in stocks than renters, which again can be interpreted as housing risk crowding out stock market risk. Generalizing to stochastic interest rates, van Hemert (2010) focuses on the interest rate exposure and choice of mortgage over the life cycle. Fischer and Stamos (2013) solve a 
life-cycle utility maximization problem assuming that expected housing returns depend on realized past returns. As in our model, Kraft and Munk (2011) allow the housing investment position to differ from housing consumption by simultaneous owning and renting (out) or by investing in house price linked financial contracts. To obtain closed-form solutions, they focus on the unrealistic unconstrained, complete market case, whereas we allow for unspanned income risk and relevant portfolio constraints. ${ }^{8}$

The above-mentioned papers involving housing assume a time-additive Cobb-Douglas utility of perishable consumption and housing consumption, but we introduce habit formation in housing consumption. Carrasco, Labeaga, and Lopez-Salido (2005), Browning and Collado (2007), and Ravina (2007) report empirical support of habit formation. Habit formation for perishable consumption has been formally studied since Ryder and Heal (1973), and habit features in (representative agent) preferences help explain asset pricing facts that seem puzzling with time-separable power utility, see, e.g., Abel (1990), Campbell and Cochrane (1999), and Menzly, Santos, and Veronesi (2004). Ingersoll (1992) and Munk (2008) add habit formation to Merton's basic one-good, no-income portfolio problem. As we do, they assume the habit is additive (utility depends on the difference between current consumption and the habit level) and multi-period (i.e., depending on the agent's full consumption history). They observe that the habit induces a buffer invested in the risk-free asset and thus reduces the optimal stock investments. The discrete-time model of Polkovnichenko (2007) includes a finite-state labor income process but assumes an additive one-period habit, i.e., the habit level is a fraction of only last period's consumption. In his benchmark setting, the optimal stock share is still large and often even 100\%. In a life-cycle model with labor income, Gomes and Michaelides (2003) shows that a multiplicative habit (utility depending on the ratio of current consumption to the habit level) leads to portfolio decisions very similar to those for standard time-additive utility.

To our knowledge the only paper with habit formation in housing consumption is by Aydilek (2013), who assumes a multiplicative one-period habit for housing in a two-good, discrete-time, life-cycle model disregarding stock investments and focusing on housing late in life. The housing habit has stronger implications in our setting because of both the multi-period and the additive specification of the habit, and we also consider the interaction between housing decisions and stock investments. Note that a good-specific or deep habit has been applied in recent equilibrium models by Ravn, Schmitt-Grohé, and Uribe (2006), van Binsbergen (2013), and Heyerdahl-Larsen (2014).

The housing habit implies a housing expenditure share that depends on the agent's age

\footnotetext{
${ }^{8}$ Other papers addressing various aspects of housing in individual decision making include Goetzmann (1993), Flavin and Yamashita (2002), Campbell and Cocco (2003), Cauley, Pavlov, and Schwartz (2007), Li and Yao (2007), Flavin and Nakagawa (2008), Attanasio, Bottazzi, Low, Nesheim, and Wakefield (2012), Chetty and Szeidl (2014), and Corradin, Fillat, and Vergara-Alert (2014).
} 
and the housing consumption relative to the habit level, which again depends on wealth and the house price. With standard Cobb-Douglas utility the share would be constant, and with CES (Constant Elasticity of Substitution) utility the share depends only on the house price. The addi-log specification used by Wachter and Yogo (2010) for the utility of basic and luxury goods implies an expenditure share depending on good prices and the level of consumption, but not directly on the agent's age as in our model, and the observed housing expenditure shares seem to exhibit an age dependence as explained in the Introduction. CES utility was assumed in the housing-extended Consumption-CAPM of Piazzesi, Schneider, and Tuzel (2007). Their key mechanism is that while agents are concerned about states with low overall consumption, they are even more concerned when the share of housing consumption is low relative to perishable consumption. This is the case for CES utility if the intratemporal elasticity of substitution between housing and non-housing consumption is larger than one. Our model shares this feature: low housing consumption is particularly bad because of the habit.

The hump-shaped life-cycle consumption profile consumption has been documented by Thurow (1969), Gourinchas and Parker (2002), and Fernández-Villaverde and Krueger (2007), among others, and is typically explained by borrowing constraints, uninsurable income risk, and mortality risk, cf., e.g., Nagatani (1972), Carroll (1997), Feigenbaum (2008), and Hansen and İmrohoroğlu (2008). Still, the hump in such models is often located around the retirement age - cf., e.g., Cocco, Gomes, and Maenhout (2005) —whereas the hump in the data is around age 50. We show that the housing habit induces a hump in perishable consumption even without constraints or risks, and that it moves the hump to an earlier age when constraints and risks are included.

As mentioned in the Introduction, Yang (2009) presents empirical evidence on housing and non-housing consumption over the life cycle. She builds a discrete-time overlapping generations equilibrium model emphasizing the role of borrowing constraints and transaction costs in producing realistic consumption patterns. Her model ignores stock investments. We incorporate the housing-stock investment decision and suggest housing habits as a mechanism to match observed life-cycle patterns in both investment and consumption.

\section{Model ingredients}

\subsection{Consumption goods}

We consider an economy with two consumption goods: perishable (or non-housing) consumption and housing consumption. The housing good is measured in a number of "units" reflecting both size, quality, and location of the residential property. For concreteness, we think of one unit as one square foot residence of average quality and location. For sim- 
plicity we refer to this good as houses. The agent consumes perishable consumption at the rate $c_{t}$ and units of housing consumption at the rate $q_{t}$.

Throughout the paper we take the perishable good to be the numeraire. The time $t$ unit price of the housing good is denoted by $H_{t}$, which varies over time as explained in the specific models in the following sections. The agent can rent housing units at a rent proportional to the price of the rented property; renting $q$ units over the time interval $[t, t+d t] \operatorname{costs} \chi q H_{t} d t$, and we refer to $\chi \geq 0$ as the rental rate.

\subsection{Preferences}

The agent develops habits for housing consumption; the habit level $\bar{q}_{t}$ satisfies

$$
\bar{q}_{t}=\bar{q}_{0} e^{-\varepsilon t}+\alpha \int_{0}^{t} e^{-\varepsilon(t-s)} q_{s} d s \quad \Rightarrow \quad d \bar{q}_{t}=\left(\alpha q_{t}-\varepsilon \bar{q}_{t}\right) d t
$$

where the initial habit level $\bar{q}_{0}$, the persistence parameter $\varepsilon$, and the scaling parameter $\alpha$ are non-negative constants. The agent derives utility from the consumption at any given time according to the habit-extended Cobb-Douglas function

$$
U(c, q, \bar{q})=\frac{1}{1-\gamma}\left[c^{b}(q-\bar{q})^{1-b}\right]^{1-\gamma},
$$

where $\gamma>1$ is a risk aversion parameter, and $b \in(0,1)$ is a weighting parameter. Note that $\gamma>1$ implies that perishable and housing consumption are substitutes in the sense that $U_{c q}<0$, and that the habit level $\bar{q}$ represents an endogenously determined subsistence level of housing consumption. If, from time $t$ on, the agent's housing consumption is exactly at the minimum, $q_{s}=\bar{q}_{s}$ for $s \geq t$, the future habit level is $\bar{q}_{u}=\bar{q}_{t} e^{-(\varepsilon-\alpha)(u-t)}$ housing units. The difference $\varepsilon-\alpha$ indicates the strength of the habit by determining the constraints the current habit level imposes on future decisions. For later use, we define the auxiliary parameters

$$
k=\frac{(1-b)(\gamma-1)}{\gamma}, \quad \hat{b}=b^{-k b /(1-b)}(1-b)^{-k} .
$$

The agent lives until time $T$. The objective of the agent at any point in time $t<T$ is to maximize $\mathrm{E}_{t}\left[\int_{t}^{T} e^{-\delta(s-t)} U\left(c_{s}, q_{s}, \bar{q}_{s}\right) d s\right]$ over the feasible consumption and investment strategies as will be specified in more detail. Here, $\delta$ is the agent's subjective time preference rate. 


\subsection{Labor income}

The individual lives until time $T$ and receives throughout life an income stream from non-financial sources at a rate of $Y_{t}$. The dynamics of $Y_{t}$ are specified in the concrete models considered in the following sections. We assume that the individual retires at a predetermined time $\tilde{T} \leq T$. At retirement the income drops to a known fraction $\Upsilon$ of the income immediately before,

$$
Y_{\tilde{T}+}=\Upsilon Y_{\tilde{T}-}
$$

This reflects the wide-spread final-salary pension schemes and is a common assumption in the literature (e.g., Cocco, Gomes, and Maenhout 2005; Lynch and Tan 2011).

\subsection{Investments, wealth, and potential constraints}

In all the models we consider the agent can invest in a risk-free asset offering a continuously compounded rate of return $r$. We assume a constant $r$ throughout to focus on the impact of housing decisions and habits on consumption and investment, and the effects of stochastic interest rates on life-cycle decisions are already well-studied. ${ }^{9}$ In the simplest model we consider, the risk-free asset is the only investment object. In the other models the agent can also invest in a single stock, representing the stock market index, and in housing units in order to capture the dual role of housing as both a consumption good and an investment object. The agent's tangible wealth at a given date is the sum of the values of her investments in the available assets. In addition, she has human wealth in terms of the present value of her future labor income.

The first two models we consider disregard any constraints on investments, and any uncertainty (also about future labor income) is assumed to be spanned by traded assets, so that markets are complete. In particular, the labor income stream can then be valued as any dividend stream and is modeled so that the human wealth at any time $t$ is of the form $Y_{t} F(t)$ for some deterministic function $F$. The total wealth of the agent is the sum of the tangible wealth $X_{t}$ and the human wealth $Y_{t} F(t)$. The agent has to make sure she can meet the minimum housing consumption defined by the habit level also in the future. As explained earlier, if the agent from time $t$ on keeps housing consumption exactly at the minimum, the future habit level is $\bar{q}_{u}=\bar{q}_{t} e^{-(\varepsilon-\alpha)(u-t)}$ housing units with a total cost rate of $\bar{q}_{u} \chi H_{u}$. The present value of these costs, which we refer to as the housing habit buffer, constitutes the amount the agent must set aside at time $t$ to cover minimum housing consumption in all future. In the models we consider, the housing habit buffer is of the form $\bar{q}_{t} \chi H_{t} B(t)$ for some deterministic function $B$. The disposable wealth of the agent at

\footnotetext{
${ }^{9}$ See, e.g., van Hemert (2010), Koijen, Nijman, and Werker (2010), Munk and Sørensen (2010), and Kraft and Munk (2011).
} 
time $t$ is thus the tangible wealth plus the human wealth less the housing habit buffer:

$$
\widehat{X}_{t}=X_{t}+Y_{t} F(t)-\bar{q}_{t} \chi H_{t} B(t) .
$$

Our main model features unspanned risks and investment constraints that are binding in some states, and then the above conclusions are invalid. But, as explained in the Introduction, we solve that decision problem by embedding it in certain unconstrained complete markets, where the above considerations hold.

\section{Two instructive models with closed-form solutions}

The full-blown model we have in mind involves unspanned risks as well as borrowing and short-selling constraints. Consequently, we are unable to solve it in closed form. To build intuition for the economic forces at play, this section considers two simpler models for which closed-form solutions are available.

\subsection{Model with full certainty}

Here we set up and solve a simple model disregarding uncertainty and any frictions. The unit house price $H_{t}$ and labor income rate develop as

$$
d H_{t}=H_{t}\left(r+\mu_{H}\right) d t, \quad d Y_{t}=Y_{t} \mu_{Y}(t) d t,
$$

so that house prices grow at a constant and labor income at a deterministic rate. The agent can only invest in the risk-free asset. Therefore, his wealth $X_{t}$ evolves as

$$
d X_{t}=r X_{t} d t+Y_{t} d t-c_{t} d t-q_{t} H_{t} \chi d t .
$$

The value function (aka. the indirect utility function) of the agent is

$$
J(t, x, h, y, \bar{q})=\sup _{c, q} \int_{t}^{T} e^{-\delta(s-t)} U\left(c_{s}, q_{s}, \bar{q}_{s}\right) d s,
$$

where $x, h, y, \bar{q}$ denote the time $t$ values of the wealth, the house price, the labor income, and the housing habit. By design, this model focuses on the effects of housing habits on consumption. In the more realistic settings considered subsequently, the value function and the optimal consumption policy have the same structure as in this simple case. 
Theorem 1 The value function is

$$
J(t, x, h, y, \bar{q})=\frac{1}{1-\gamma}(\chi h)^{\gamma k} G(t)^{\gamma}(x+y F(t)-\bar{q} \chi h B(t))^{1-\gamma},
$$

where

$$
\begin{gathered}
B(t)=\frac{1}{r_{B}}\left(1-e^{-r_{B}(T-t)}\right), \\
G(t)=\hat{b} \int_{t}^{T} e^{-r_{G}(s-t)}(1+\alpha B(s))^{k} d s, \\
F(t)= \begin{cases}\int_{t}^{T} e^{-\int_{t}^{u} r_{F}(s) d s} d u, & t \in[\tilde{T}, T], \\
\int_{t}^{\tilde{T}} e^{-\int_{t}^{u} r_{F}(s) d s} d u+\Upsilon \int_{\tilde{T}}^{T} e^{-\int_{t}^{u} r_{F}(s) d s} d u, & t<\tilde{T}, \\
r_{G}=\frac{\delta}{\gamma}+\frac{\gamma-1}{\gamma} r-\left(r+\mu_{H}\right) k, \quad r_{F}(t)=r-\mu_{Y}(t), & r_{B}=\varepsilon-\alpha-\mu_{H} .\end{cases}
\end{gathered}
$$

With $\widehat{X}_{t}$ defined in (4), the optimal perishable and housing consumption rates are

$$
c_{t}=b \hat{b} \frac{(1+\alpha B(t))^{k}}{G(t)} \widehat{X}_{t}, \quad q_{t}=\bar{q}_{t}+(1-b) \hat{b} \frac{(1+\alpha B(t))^{k-1}}{\chi H_{t} G(t)} \widehat{X}_{t} .
$$

The life-cycle profile of perishable consumption is hump shaped if $r>\delta, \mu_{H}<\varepsilon$, and

$$
k\left[\frac{\alpha r_{B}}{\left(\alpha+r_{B}\right) e^{r_{B} T}-\alpha}-\left(r+\mu_{H}\right)\right] \leq \frac{r-\delta}{\gamma} \leq k\left(\alpha-\left[r+\mu_{H}\right]\right) .
$$

Optimal perishable and housing consumption are related via

$$
c_{t}=\frac{b}{1-b}\left(q_{t}-\bar{q}_{t}\right) \chi H_{t}(1+\alpha B(t)),
$$

and the housing expenditure share is

$$
\frac{\chi q_{t} H_{t}}{c_{t}+\chi q_{t} H_{t}}=\frac{1-b}{1-b+b\left(1-\frac{\bar{q}_{t}}{q_{t}}\right)(1+\alpha B(t))} .
$$

Without habit formation $\left(\bar{q}_{t}=0, \alpha=0\right)$, perishable consumption is a constant multiple of housing consumption expenditures, and the housing expenditure share is the constant $1-b$. Furthermore, the growth rate in perishable consumption is the constant $[r-\delta+$ $\left.(1-b)(\gamma-1)\left(r+\mu_{H}\right)\right] / \gamma$. With $r>\delta, \gamma>1$, and a non-negative house price growth rate $r+\mu_{H}$, perishable consumption is increasing throughout life.

The housing habit implies a time-dependent perishable-housing consumption ratio. Eq. (10) shows that $\chi H_{t}(1+\alpha B(t))$ is the effective time $t$ unit price of housing consump- 
tion. Since $B$ is decreasing over time, so is the effective price of housing, other things equal. Housing consumption is relatively more expensive for young agents: through the housing habit, the consumption of one extra unit of housing costs not only the current rent but also future required rents due to the increase in the habit level. The present value of the habit-induced required future rents is larger for young than for old agents. Since perishable goods and housing services are substitutes, the optimal composition of consumption is tilted towards perishable goods when young and towards housing when old. Embedding this mechanism in a setting where overall consumption is optimally increasing over life can lead to a hump-shaped life-cycle pattern in perishable consumption as seen in the data. With housing habits, the housing expenditure share is time dependent, and it decreases with $q_{t}$ and thus with disposable wealth $\widehat{X}_{t}$. The housing expenditure share is therefore higher in hard times than in good times, as seen in the data.

Figure 3 illustrates the optimal consumption pattern over a 50-year period that could represent the situation of a 30-year old expecting to retire at age 60 and living on until age 80. Initial wealth and (after-tax) income are set to 20 -representing $\$ 20,000$-in line with the median net worth and before-tax income statistics for young individuals according to the 2013 Survey of Consumer Finances. ${ }^{10}$ The left panel shows consumption expenditures (in thousands of dollars, per year) with perishable consumption exhibiting a clear hump for the case of housing habits unlike the no-habit case. The habit assumed here is relatively weak in the sense that $\varepsilon-\alpha=0.1$ so that as long as the agent consumes housing at the minimum, this minimum decreases by $10 \%$ per year. Optimal housing expenditures are increasing early in life, then flattens out, and then increasing again in the final years. The housing expenditure share shown in the right panel exhibits the same shape as seen in the data, cf. Figure 2.

Mortality risk is known to affect the life-cycle profile of consumption and can generate a hump. In Appendix B we extend the above model to mortality risk and bequest. Following Richard (1975), Blanchard (1985), and others, we assume the agent can buy or sell life insurance. The mortality intensity is given by the deterministic function $\zeta(t)$. The agent can obtain a life insurance contract that pays a continuous flow at the rate of $\Gamma \zeta(t) N_{t}$ per year to the agent, but in return the issuer receives the amount $N_{t}$ from the estate of the agent upon death. Here $N_{t}$ is a choice variable of the agent and can be positive or negative. The agent's optimal strategy is then similar to the no-mortality case but

\footnotetext{
${ }^{10}$ The Survey of Consumer Finances is conducted by the Federal Reserve Board, see http://www . federalreserve.gov/econresdata/scf/scfindex.htm. Summary results are presented in "Changes in U.S. Family Finances from 2010 to 2013: Evidence from the Survey of Consumer Finances" published in the September 2014 issue of the Federal Reserve Bulletin and available on the above homepage. According to Table 1 of this document, the before-tax median family income was $\$ 35.300$ for age (of family head) less than 35 and $\$ 60.900$ for age 35-44. From Table 2, the median net worth per family was $\$ 10.400$ for age less than 35 and $\$ 46.700$ for age 35-44. Our numbers reflect after-tax income and wealth per adult.
} 

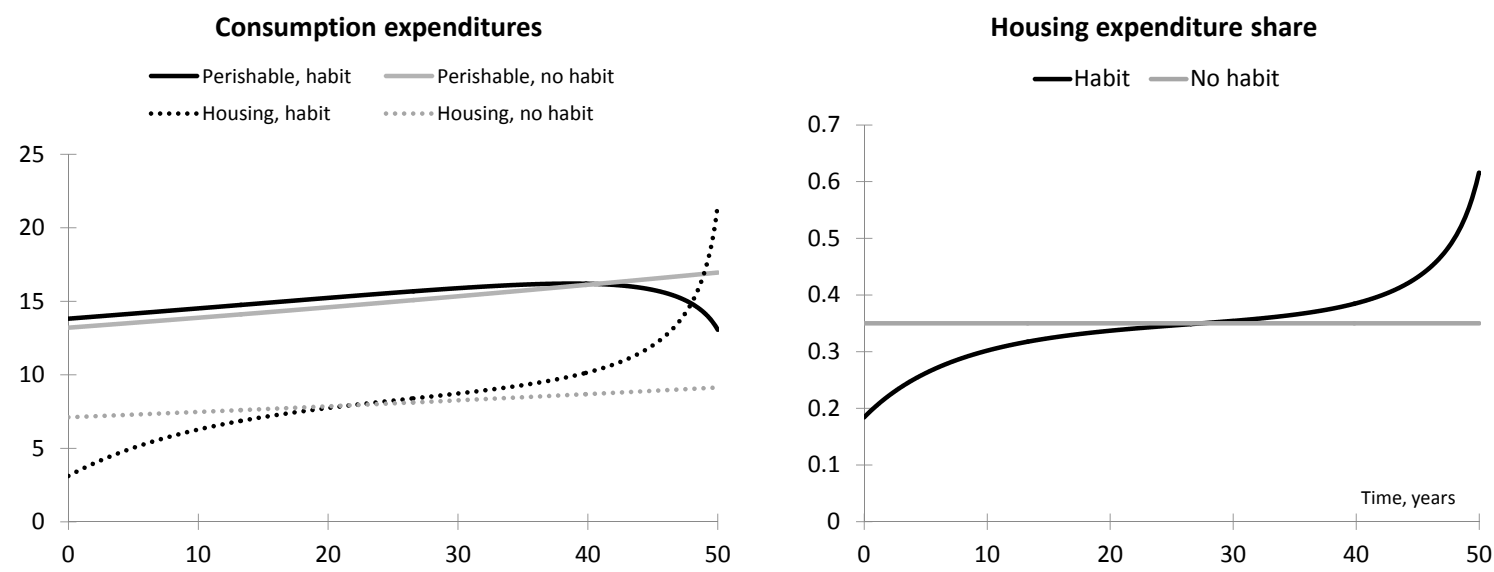

Figure 3: Consumption in the deterministic model. The left panel illustrates the expenditures on perishable goods and housing and the right panel illustrates the housing expenditure share, both with and without a housing habit. The graphs are generated using the deterministic model of Section 4.1. The parameter values are: $\delta=0.02, \gamma=2$, $t=0, \tilde{T}=30, T=50, X_{0}=20, Y_{0}=20, \mu_{Y}=0.01, \Upsilon=0.6, r=0.03, \chi=0.06$, $H_{0}=0.25, \mu_{H}=-0.03$. Without habit formation we put $b=0.65$. With habit formation we let $\bar{q}_{0}=150, \alpha=0.8, \varepsilon=0.9$, and $b=0.69$ (to obtain the same average housing expenditure share as in the no-habit case).

with the (deterministically increasing) mortality intensity $\zeta(t)$ added to the constant time preference $\delta$, so that the agent effectively becomes more impatient with age. We assume a bequest utility of the form $w^{\gamma} \frac{1}{1-\gamma} X_{\tau}^{1-\gamma}$, where $\tau$ is the time of death, $X_{\tau}$ is the bequeathed wealth (after adjusting for an insurance payment upon death), and $w$ is the preference weight of bequest. We now assume the agent is initially 30 years, retires at 65 , and lives on to at most 100 years applying throughout the mortality rates implied by the life tables for the total U.S. population as of $2009 .^{11}$ Figure 4 depicts perishable consumption over the life cycle with mortality risk. The panels differ in the preference for bequest with $w=1$ in the left panel and $w=10$ in the right panel; if surviving until 100, an agent with the weak habit, $\varepsilon-\alpha=0.1$, leaves a wealth of 26.5 if $w=1$ and 105.1 if $w=10$. Each panel shows the consumption profile for the no-habit case, a weak habit $(\varepsilon-\alpha=0.1$ as above), and a strong habit $(\varepsilon-\alpha=0.02)$. The diamonds indicate the location of the hump. Without habit formation mortality risk induces a consumption hump rather late in life, but habit formation produces an earlier hump more in line with the observed hump. In particular, with the relatively strong habit our simple model generates a hump at age 50 (age 55) with the large (small) bequest weight.

Habit formation also impacts the sensitivity of consumption with respect to changes in wealth or income. The ratio $\frac{\partial c_{t}}{\partial \widehat{X}_{t}} / \frac{\partial\left(\chi q_{t} H_{t}\right)}{\partial \widehat{X}_{t}}$ of the marginal propensities to consume

\footnotetext{
${ }^{11}$ Published at the Centers for Disease Control and Prevention under the U.S. Department of Health and Human Services, see http://www.cdc.gov/nchs/data/nvsr/nvsr62/nvsr62_07.pdf.
} 
Small bequest weight

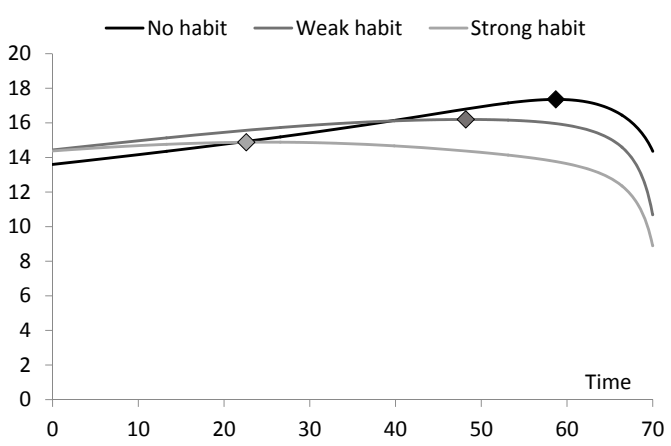

Large bequest weight

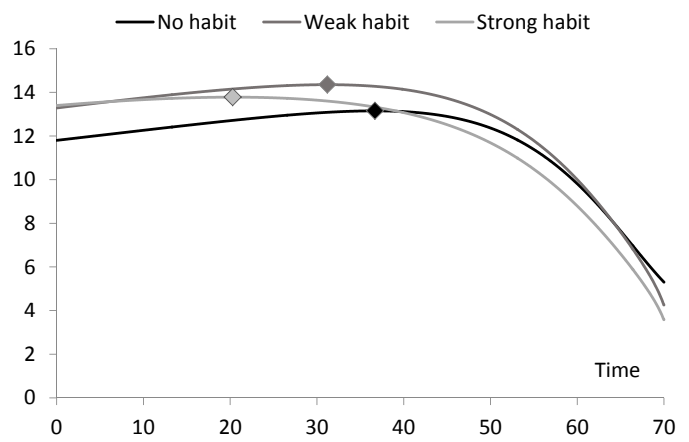

Figure 4: Perishable consumption with mortality risk and bequest. Each panel shows perishable consumption over the life cycle without habit, with a weak habit $(\alpha=$ $0.8, \varepsilon=0.9)$, and with a strong habit $(\alpha=0.88, \varepsilon=0.9)$. The graphs are generated using the extension of the deterministic model to bequest and mortality risk, which is presented in Appendix B.1. The left panel assumes a bequest utility parameter of $w=1$, whereas the right panel assumes $w=10$. For both panels the following parameter values are used: $\delta=0.01, \gamma=5, t=0, \tilde{T}=35, T=70, X_{0}=20, Y_{0}=20, \mu_{Y}=0.01, \Upsilon=0.6, r=0.03$, $\chi=0.06, H_{0}=0.25, \mu_{H}=-0.03, \Gamma=1.5$. Without habit formation we put $b=0.65$. With habit formation we let $b=0.69$ and $\bar{q}_{0}=150$.

out of disposable wealth equals $(1+\alpha B(t)) b /(1-b)$, which is constant in the no-habit case, but bigger and time-dependent in the case with habits. With the parameters used to generate Figure 3, the ratio is 1.9 in the no-habit case, whereas it declines from 15.9 initially to 2.3 at the end of life with the weak habit and from 38.2 to 2.3 with the strong habit. A young agent being hit by a negative wealth or income shock reduces perishable consumption expenditures much more than housing expenditures in the habit model, which seems better in accordance with data.

\subsection{A model with spanned risks and no frictions}

Next we extend the simplistic model considered above by adding a risky investment asset (the stock index) and uncertainty about both the house price, the income, and the stock price. The agent can now also invest in housing units which then serve both a consumption and investment purpose. The individual can own any non-negative number of housing units and can simultaneously rent out some of the owned housing units or rent additional housing units. In this way the individual can disentangle the consumption of housing units from the investment position in the housing market.

To obtain a closed-form solution, we assume the agent can continuously adjust the number of units rented and the number of units owned without transaction costs. Observed changes in the physical ownership of housing units seem rare and costly, but the remodeling or the extension of a house also constitutes an increase in the number of housing units 
owned due to the higher quality or increased space. In addition, individuals can indirectly invest in housing units by purchasing shares in residential REITs (Real Estate Investment Trusts), or even exchange-traded funds emulating the REIT market, or in other financial assets closely linked to house prices such as the Case-Shiller derivatives. ${ }^{12}$ Hence, home owners can implement short-term variations in their desired housing investment position by remodeling or investing in REITs, whereas they might prefer implementing larger changes in both desired housing consumption and investment through (rare) physical transactions of housing units. A pure housing investment position is obtained by owning housing units and renting them out either directly or through REITs. We assume ownership entails maintenance costs (could include property taxes) equal to a constant fraction $m \geq$ 0 of the property value. Hence the return on a pure investment in a housing unit is $d H_{t}+(\chi-m) H_{t} d t$ over the $d t$-interval following time $t$.

In this section we deliberately abstract from any portfolio constraints and assume that all risks are spanned by traded assets. ${ }^{13}$ More precisely, the stock price $S_{t}$ (including reinvested dividends), the unit house price $H_{t}$, and the labor income rate $Y_{t}$ satisfy

$$
\begin{aligned}
& d S_{t}=S_{t}\left[\left(r+\mu_{S}\right) d t+\sigma_{S} d W_{S t}\right] \\
& d H_{t}=H_{t}\left[\left(r+\mu_{H}\right) d t+\sigma_{H}\left(\rho_{H S} d W_{S t}+\sqrt{1-\rho_{H S}^{2}} d W_{H t}\right)\right], \\
& d Y_{t}=Y_{t}\left[\mu_{Y}(t) d t+\sigma_{Y}(t)\left(\rho_{Y S} d W_{S t}+\sqrt{1-\rho_{Y S}^{2}} d W_{H t}\right)\right],
\end{aligned}
$$

where $W_{S}$ and $W_{H}$ are independent standard Brownian motions. We define $\tilde{\rho}_{H Y}=$ $\sqrt{1-\rho_{Y S}^{2}} / \sqrt{1-\rho_{H S}^{2}}$ and note that the house-income correlation is $\rho_{H Y}=\rho_{H S} \rho_{Y S}-$ $\tilde{\rho}_{H Y}\left(1-\rho_{H S}^{2}\right)$. As there is no income-specific shock term, the agent can adjust his exposure to both stock price, house price, and income shocks through his positions in the stock and the housing units. The excess expected return $\mu_{S}$ and the volatility $\sigma_{S}$ of the stock are constant. Similarly, the excess expected house price growth rate $\mu_{H}$ and volatility $\sigma_{H}$ are constants, as are the stock-house price correlation $\rho_{H S}$ and the stock-income correlation $\rho_{Y S}$. The instantaneous Sharpe ratios on investments in stocks and houses are then

$$
\lambda_{S}=\frac{\mu_{S}}{\sigma_{S}}, \quad \lambda_{H}=\frac{\mu_{H}+\chi-m}{\sigma_{H}} .
$$

\footnotetext{
${ }^{12}$ Well-developed REIT markets exist in many countries. Cotter and Roll (2014) study the risk and return characteristics of U.S. REITs. As explained by Ang (2014, Ch. 11), REIT returns exhibit only a low short-run correlation with returns on directly owned real estate (and a higher correlation with common stocks), but longer-term correlations are significantly higher, cf., e.g., Hoesli and Oikarinen (2012). Pagliari, Scherer, and Monopoli (2005) argue that after various relevant adjustments REIT returns and direct real estate returns are much more highly correlated even in the short run, and Lee, Lee, and Chiang (2008) and others report that REITs behave more and more like real estate and less and less like ordinary stocks.

${ }^{13}$ This model extends Kraft and Munk (2011) to housing habits (they allow stochastic interest rates).
} 
Both the expected growth rate and volatility of income may depend on the individual's age. Where most life-cycle papers assume a constant retirement income, we allow for risk. This is motivated by (i) some retirees continue to earn income from proprietary businesses or other non-traded assets; (ii) uncertainty about medical expenses implies that the disposable income is risky (see De Nardi, French, and Jones 2010); (iii) because of mortality risk, the individual may miss retirement payments and, while we do not model mortality formally, retirement income risk captures this effect parsimoniously.

Let $\Pi_{S t}$ be the fraction of tangible wealth invested in the stock, and let $\phi_{o t}$ and $\phi_{r t}$ denote the housing units owned (physically or through REITs) and rented. Then the units of housing consumption and the fraction of tangible wealth invested in housing are

$$
q_{t} \equiv \phi_{o t}+\phi_{r t}, \quad \Pi_{H t} \equiv \frac{\phi_{o t} H_{t}}{X_{t}}
$$

respectively. The wealth invested in the risk-free asset is residually determined as $M_{t}=$ $X_{t}\left(1-\Pi_{S t}-\Pi_{H t}\right)$. The dynamics of tangible wealth are

$$
\begin{aligned}
d X_{t}= & \Pi_{S t} X_{t} \frac{d S_{t}}{S_{t}}+M_{t} r d t+\phi_{o t}\left(d H_{t}-m H_{t} d t\right)-\phi_{r t} \chi H_{t} d t-c_{t} d t+Y_{t} d t \\
= & \left(X_{t}\left[r+\Pi_{S t} \mu_{S}+\Pi_{H t}\left(\mu_{H}+\chi-m\right)\right]+Y_{t}-c_{t}-q_{t} \chi H_{t}\right) d t \\
& +X_{t}\left(\Pi_{S t} \sigma_{S}+\Pi_{H t} \sigma_{H} \rho_{H S}\right) d W_{S t}+X_{t} \Pi_{H t} \sigma_{H} \sqrt{1-\rho_{H S}^{2}} d W_{H t} .
\end{aligned}
$$

The value function is now defined as

$$
J(t, x, h, y, \bar{q})=\sup _{c, q, \Pi_{S}, \Pi_{H}} \mathrm{E}_{t}\left[\int_{t}^{T} e^{-\delta(s-t)} U\left(c_{s}, q_{s}, \bar{q}_{s}\right) d s\right]
$$

Theorem 2 The value function is given by (5)-(8) with

$$
\begin{aligned}
r_{G} & =\frac{\delta}{\gamma}+\frac{\gamma-1}{\gamma} r+\frac{\gamma-1}{2 \gamma^{2}} \Lambda^{2}-k\left(r+\frac{\mu_{H}}{\gamma}-\frac{\gamma-1}{\gamma}(\chi-m)+\frac{1}{2}(k-1) \sigma_{H}^{2}\right), \\
r_{F}(t) & =r-\mu_{Y}(t)+\sigma_{Y}(t)\left(\lambda_{S} \rho_{Y S}+\tilde{\rho}_{H Y}\left[\lambda_{H}-\rho_{H S} \lambda_{S}\right]\right), \\
r_{B} & =\varepsilon-\alpha+\chi-m \\
\Lambda^{2} & =\lambda_{S}^{2}+\frac{1}{1-\rho_{H S}^{2}}\left(\lambda_{H}-\rho_{H S} \lambda_{S}\right)^{2} .
\end{aligned}
$$

With disposable wealth $\widehat{X}_{t}$ defined in (4), the optimal consumption decisions are still given by (9), and the optimal investment strategy is

$$
\Pi_{S t}=\frac{1}{\gamma} \frac{\lambda_{S}-\rho_{H S} \lambda_{H}}{\sigma_{S}\left(1-\rho_{H S}^{2}\right)} \frac{\widehat{X}_{t}}{X_{t}}-\frac{\sigma_{Y}(t)}{\sigma_{S}}\left(\rho_{Y S}-\rho_{H S} \tilde{\rho}_{H Y}\right) \frac{Y_{t} F(t)}{X_{t}},
$$




$$
\Pi_{H t}=\frac{1}{\gamma} \frac{\lambda_{H}-\rho_{H S} \lambda_{S}}{\sigma_{H}\left(1-\rho_{H S}^{2}\right)} \frac{\widehat{X}_{t}}{X_{t}}-\frac{\sigma_{Y}(t)}{\sigma_{H}} \tilde{\rho}_{H Y} \frac{Y_{t} F(t)}{X_{t}}+k \frac{\widehat{X}_{t}}{X_{t}}+\bar{q}_{t} \chi B(t) \frac{H_{t}}{X_{t}} .
$$

The expected consumption $\mathrm{E}\left[c_{t}\right]$ is a hump-shaped function of time if $\varepsilon+\chi-m>0$ and

$$
\frac{k \alpha r_{B}}{\left(\alpha+r_{B}\right) e^{r_{B} T}-\alpha}<K_{c}<k \alpha
$$

and then the hump occurs at $t=t_{\text {hump }}$, where

$$
\begin{aligned}
K_{c} & =\frac{r-\delta}{\gamma}+\frac{\gamma+1}{2 \gamma^{2}} \Lambda^{2}+k\left(r+\mu_{H}+\frac{\mu_{H}+\chi-m}{\gamma}+\frac{k-1}{2} \sigma_{H}^{2}\right), \\
t_{\text {hump }} & =T-\frac{1}{r_{B}} \ln \left(\frac{\alpha\left(K_{c}+k r_{B}\right)}{K_{c}\left(\alpha+r_{B}\right)}\right) .
\end{aligned}
$$

The value function and the optimal consumption policy are thus similar to the fullcertainty model, only the "discount rates" $r_{F}$ and $r_{G}$ are adjusted to account for risk premia. The housing habit can generate a hump in expected perishable consumption. The optimal stock investment in (17) is the sum of the standard speculative demand determined by the current risk-return tradeoff and an income-adjustment term. In the optimal housing investment the first two terms have a similar interpretation, but there are two additional terms. The third term in (18) represents a hedge against increases in the price of housing consumption, which is accomplished by having a higher wealth exposure to house prices, cf. Sinai and Souleles (2005). The final term ensures that the agent can reach at least the minimum housing consumption in the remaining life time. As the costs of ensuring that the minimum is achieved vary with house prices, this calls for an increased wealth exposure to house prices. Both the hedge term and the habit-insurance term are thus positive. The income-adjustment terms undo the extent to which the human capital resembles an investment in the stock or housing units.

The presence of habit formation lowers the disposable wealth $\widehat{X}_{t}$ through the housing habit buffer $\bar{q}_{t} \chi H_{t} B(t)$, which is typically decreasing as the remaining life time shrinks. Therefore, the habit reduces the optimal stock investment, in particular for young investors. The habit also reduces the speculative housing demand and the house price hedge demand but, on the other hand, induces a positive habit-insurance term so that the net effect is parameter dependent.

For young individuals the human wealth is typically significantly higher than the financial wealth so that the ratio $\widehat{X}_{t} / X_{t}$ can be bigger than one. Consequently, unless the agent is very risk averse or the income is relatively stock-like, the total stock demand is large, and a $100 \%$ upper limit on the stock weight can easily bind. Constraints on the housing investment might also bind. 
Life-cycle variations in investments enter through three channels. First, the direct age-dependence of human wealth (via the function $F(t)$ ), which is typically decreasing over life as the remaining working period shrinks. ${ }^{14}$ Secondly, as the remaining life time shortens, the housing habit buffer $\bar{q}_{t} \chi H_{t} B(t)$ generally decreases, which carries over to the last component of the housing investment demand.Thirdly, the optimal decisions typically lead to a hump-shaped pattern in tangible wealth $X_{t}$ over the life cycle with net savings during the working phase and consumption out of savings in retirement. This induces an age-dependence in all of the terms in the portfolio weights.

\section{The full-fledged model}

\subsection{Model specification}

Our main model differs from the model considered in Section 4.2 by having unspanned labor income risk and investment constraints. Instead of (12), the income rate follows

$$
d Y_{t}=Y_{t}\left[\mu_{Y}(t) d t+\sigma_{Y}(t)\left(\rho_{Y S} d W_{S t}+\hat{\rho}_{Y H} d W_{H t}+\hat{\rho}_{Y} d W_{Y t}\right)\right]
$$

where $W_{Y}=\left(W_{Y t}\right)$ is a standard Brownian motion independent of $W_{S}$ and $W_{H}$. Unless $\hat{\rho}_{Y}=0$, the income risk is unspanned by traded assets so the individual faces an incomplete market. The income-house and income-stock correlations $\rho_{H Y}$ and $\rho_{S Y}$ are constant, and

$$
\hat{\rho}_{Y H} \equiv \frac{\rho_{Y H}-\rho_{Y S} \rho_{H S}}{\sqrt{1-\rho_{H S}^{2}}}, \quad \hat{\rho}_{Y} \equiv \sqrt{1-\rho_{Y S}^{2}-\hat{\rho}_{Y H}^{2}} .
$$

Since income contains unspanned risk and is not bounded from below by a positive level, non-negative terminal wealth can only be insured by keeping tangible wealth nonnegative throughout, i.e., $X_{t} \geq 0$ for all $t \in[0, T]$. Furthermore, we impose the constraints

$$
\Pi_{S} \geq 0, \quad \Pi_{H} \geq 0, \quad \Pi_{S}+\kappa \Pi_{H} \leq 1,
$$

which rule out short-selling and limits borrowing to a fraction $(1-\kappa)$ of the current value of the housing investment, where $\kappa \in[0,1]$.

An admissible strategy $a=\left(c, q, \Pi_{S}, \Pi_{H}\right)$ satisfies standard integrability conditions and the above constraints, and it generates the expected utility

$$
J(t, x, y, h, \bar{q} ; a)=E_{t}\left[\int_{t}^{T} e^{-\delta(s-t)} U\left(c_{s}, q_{s}, \bar{q}_{s}\right) d s\right]
$$

\footnotetext{
${ }^{14}$ With a sufficiently high income growth rate, the human wealth can be locally increasing with age, but eventually it decreases.
} 
where the expectation is conditional on $X_{t}=x, Y_{t}=y, H_{t}=h$, and $\bar{q}_{t}=\bar{q}$. If $\mathcal{A}$ denotes the set of all admissible strategies, the value function is defined as

$$
J(t, x, y, h, \bar{q})=\sup _{a \in \mathcal{A}} J(t, x, y, h, \bar{q} ; a)
$$

Because of incomplete markets and portfolio constraints, we cannot solve the problem in closed form. Due to the high number of state variables, grid-based methods are cumbersome to implement and lead to high computation times when used with the grid sizes required for high precision. Below we outline the numerical solution method we apply.

\subsection{Outline of the solution approach}

We apply the so-called SAMS (Simulation of Artificial Markets Strategies) approach introduced by Bick, Kraft, and Munk (2013), henceforth BKM, and illustrated in Figure 5. The method exploits that in each of various artificial markets a closed-form solution to the utility maximization problem exists; the solution is very similar to those presented in Theorems 1 and 2. ${ }^{15}$ In any of the artificial markets the agent is unconstrained, has access to the same assets (with identical or higher returns) as in the true market, plus an additional asset completing the market. Hence, the agent can achieve at least as high an expected utility as in the true market. In the figure, the points marked to the right on the axis indicate the maximal utility in different artificial markets denoted by $\theta_{1}, \theta_{2}$, etc. The lowest expected utility among these artificial markets - indicated by $\bar{\theta}$ on the axis - is still at least as large as the unknown maximum in the true market.

The explicit, optimal strategy in an artificial market is infeasible in the true market, but can be feasibilized - transformed into a feasible strategy in the true market - and its expected utility in the true market can be evaluated by Monte Carlo simulation. In this way we can generate the points on the left part of the axis in Figure 5. Maximizing over these feasibilized strategies, we obtain the expected utility indicated by $\theta^{*}$ in the figure. The corresponding near-optimal strategy is the strategy suggested by the SAMS approach.

As with other numerical methods, the suggested strategy is unlikely to be identical to the truly optimal strategy so the agent suffers a welfare loss by applying the suggested strategy. We derive an upper bound on the welfare loss by comparing the expected utility generated by the near-optimal strategy to the expected utility in the worst of the artificial markets considered. This upper bound represents a measure of precision of the approach. In wealth-equivalent terms, the loss bound typically corresponds to less than two percent of the agent's wealth. In the examples of BKM, the true loss is significantly smaller than

\footnotetext{
${ }^{15}$ Artificial markets were introduced by Karatzas, Lehoczky, Shreve, and Xu (1991) and Cvitanić and Karatzas (1992).
} 


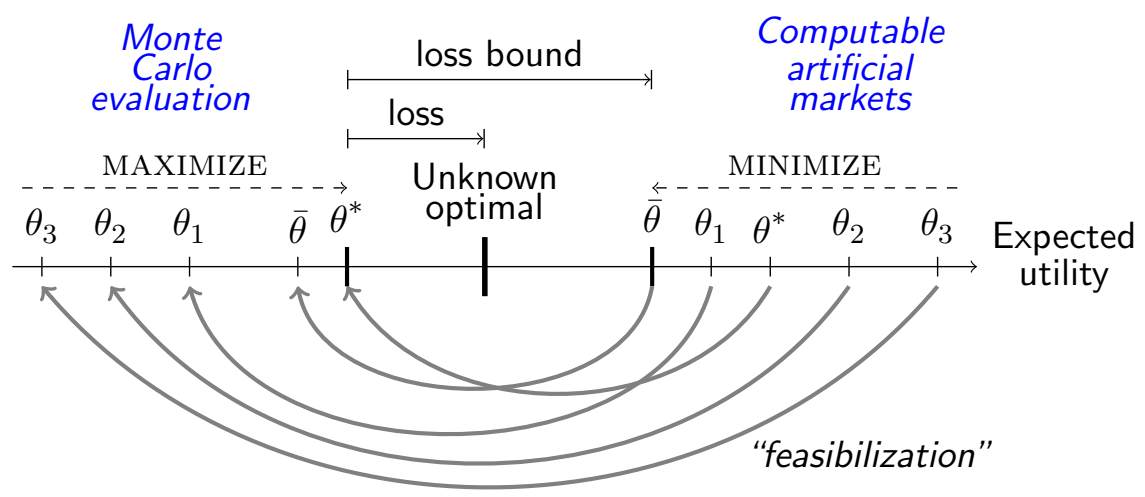

Figure 5: Our solution technique. The axis shows the agent's expected utility. "Unknown optimal" represents the indirect utility in the true market, i.e., the expected utility generated by the unknown optimal consumption-investment strategy. Each point to the right corresponds to the indirect utility in an artificial market with deterministic modifiers characterized by some parameter set $\theta$. The corresponding strategy is transformed into a feasible strategy in the true market which generates an expected utility on the left part of the axis. The best of these strategies is derived from the optimal strategy in an artificial market for some $\theta^{*}$. The arrows above the axis indicate the unknown utility loss and a computable upper bound on the loss the agent suffers by following the best of the considered feasible strategies instead of the unknown optimal strategy.

the loss bound, and we expect the same in our model.

Relative to alternative numerical methods, this approach distinguishes itself by being relatively easy to implement, being based on closed-form consumption and investment strategies, and providing a measure of its accuracy. The following subsections provide details on the SAMS method applied to our problem.

\subsection{A family of artificial markets}

Intuitively we search for artificial markets in which (i) we can solve the utility maximization problem and (ii) the optimal consumption-investment strategy is close to what we expect to be a good, feasible strategy in the true market. Because the portfolio weights in an artificial market are unconstrained, whereas they must satisfy (23) in the true market, we adjust the risk-free rate or the expected excess returns on stocks and houses. Following Cvitanić and Karatzas (1992) and BKM (Sec. 8), and using $\nu^{-}=\max (-\nu, 0)$, we define

$$
\tilde{\mu}_{S}(t)=\mu_{S}+\nu_{S}(t), \quad \tilde{\mu}_{H}(t)=\mu_{H}+\nu_{H}(t), \quad \tilde{r}(t)=r+\max \left(\nu_{S}(t)^{-}, \frac{1}{\kappa} \nu_{H}(t)^{-}\right) .
$$

The dynamics of stock and house prices in the artificial market are thus

$$
\begin{aligned}
d \tilde{S}_{t} & =\tilde{S}_{t}\left[\left(\tilde{r}(t)+\tilde{\mu}_{S}(t)\right) d t+\sigma_{S} d W_{S t}\right], \tilde{S}_{0}=S_{0} \\
d \tilde{H}_{t} & =\tilde{H}_{t}\left[\left(\tilde{r}(t)+\tilde{\mu}_{H}(t)\right) d t+\sigma_{H}\left(\rho_{H S} d W_{S t}+\sqrt{1-\rho_{H S}^{2}} d W_{H t}\right)\right], \tilde{H}_{0}=H_{0} .
\end{aligned}
$$


Irrespective of the signs and magnitudes of $\nu_{S}$ and $\nu_{H}$, the risk-free rate and the expected returns of stocks and houses are at least as big in the artificial as in the true market.

To complete the market we introduce an income derivative, i.e., an asset sensitive to the income-specific shock $W_{Y}$. The price dynamics of this asset are of the form

$$
d I_{t}=I_{t}\left[\left(\tilde{r}(t)+\nu_{I}(t)\right) d t+\rho_{I S} d W_{S t}+\hat{\rho}_{I H} d W_{H t}+\hat{\rho}_{I} d W_{Y t}\right]
$$

where

$$
\hat{\rho}_{I H} \equiv \frac{\rho_{I H}-\rho_{I S} \rho_{H S}}{\sqrt{1-\rho_{H S}^{2}}}, \quad \hat{\rho}_{I} \equiv \sqrt{1-\rho_{I S}^{2}-\hat{\rho}_{I H}^{2}},
$$

and we require $\hat{\rho}_{I}>0$. The assumption of a unit volatility is without loss of generality. The asset is characterized by the excess expected return $\nu_{I}(t)$ and the correlations $\rho_{I S}, \rho_{I H}$. Let $\Pi_{I t}$ be the fraction of tangible wealth invested in this asset.

The housing consumption constitutes a challenge not covered by the single-good case in which BKM introduced the SAMS approach. The house prices in the artificial market and the true market are related through

$$
\tilde{H}_{t}=\omega(t) H_{t}, \quad \omega(t)=e^{\int_{0}^{t} r_{\omega}(u) d u}, \quad r_{\omega}(u)=\nu_{H}(u)+\max \left(\nu_{S}(u)^{-}, \frac{1}{\kappa} \nu_{H}(u)^{-}\right) \geq 0 .
$$

Hence, if we let the rental rate be the same as in the true market, the consumption of a given number of housing units would be more expensive (and thus maybe not feasible) in the artificial market. Therefore we set the rental rate and the maintenance rate in $\mathcal{M}_{\theta}$ to

$$
\tilde{\chi}(t)=\frac{\chi}{\omega(t)}, \quad \tilde{m}(t)=m+\tilde{\chi}(t)-\chi
$$

which sustains the unit rental price $\tilde{\chi}(t) \tilde{H}_{t}=\chi H_{t}$, as well as the net rental rate after maintenance costs, $\tilde{\chi}(t)-\tilde{m}(t)=\chi-m$. The Sharpe ratios of the stock and the house investment (including the net rental rate) are

$$
\tilde{\lambda}_{S}(t)=\frac{\tilde{\mu}_{S}(t)}{\sigma_{S}}, \quad \tilde{\lambda}_{H}(t)=\frac{\tilde{\mu}_{H}(t)+\chi-m}{\sigma_{H}},
$$

while $\nu_{I}(t)$ is the Sharpe ratio of the income derivative.

An artificial market $\mathcal{M}_{\theta}$ corresponds to a given choice of

$$
\theta=\left(\nu_{S}(t), \nu_{H}(t), \nu_{I}(t), \rho_{I S}, \rho_{I H}\right)
$$

Let $J_{\theta}(t, x, y, h, \bar{q})$ denote the value function in the artificial market $\mathcal{M}_{\theta}$, that is the expected utility of the remaining life maximized over all strategies $\left(c, q, \Pi_{S}, \Pi_{H}, \Pi_{I}\right)$. In this 
market the expected utility of any strategy is still determined by (24), but the relevant dynamics are now given by (3), (22), (25), (26), and (27). We verify in Appendix A.3 that any strategy admissible in the true market leads to at least the same utility in each artificial market. Since the set of admissible strategies in the artificial complete market is greater than in the incomplete market, the next lemma follows.

Lemma 1 For any artificial market $\mathcal{M}_{\theta}$, the following inequality holds:

$$
J_{\theta}(t, x, y, h, \bar{q}) \geq J(t, x, y, h, \bar{q})
$$

Let $\Theta$ represent the set of $\theta$ 's where $\nu_{S}(t), \nu_{H}(t), \nu_{I}(t)$ are deterministic. For $\theta \in \Theta$ we derive below a closed-form expression for $J_{\theta}$ and the corresponding optimal strategy. Minimizing over $\theta \in \Theta$, we get an upper bound on the true-market value function:

$$
\bar{J}(t, x, y, h, \bar{q})=\min _{\theta \in \Theta} J_{\theta}(t, x, y, h, \bar{q})
$$

\subsection{Optimal decisions in the artificial markets}

Any artificial market $\mathcal{M}_{\theta}, \theta \in \Theta$, is very similar to the market considered in Section 4.2, and therefore the value function and optimal strategies have the same form as found in that model. The only differences are that there are now three risky assets instead of two, and that various quantities are now time dependent.

Theorem 3 For $\theta \in \Theta$, the value function in the artificial market $\mathcal{M}_{\theta}$ is

$$
J_{\theta}(t, x, y, h, \bar{q})=\frac{1}{1-\gamma}(\chi h)^{k \gamma} G_{\theta}(t)^{\gamma}\left(x+y F_{\theta}(t)-\bar{q} \chi h B_{\theta}(t)\right)^{1-\gamma}
$$

where

$$
\begin{aligned}
& G_{\theta}(t)=\hat{b} \int_{t}^{T} e^{-\int_{t}^{u} r_{G}(s) d s}\left(1+\alpha B_{\theta}(u)\right)^{k} d u, \\
& F_{\theta}(t)= \begin{cases}\int_{t}^{T} e^{-\int_{t}^{u} r_{F}(s) d s} d u & t \in(\tilde{T}, T], \\
\int_{t}^{\tilde{T}} e^{-\int_{t}^{u} r_{F}(s) d s} d u+\Upsilon \int_{\tilde{T}}^{T} e^{-\int_{t}^{u} r_{F}(s) d s} d u & t \in[0, \tilde{T}],\end{cases} \\
& B_{\theta}(t)=\int_{t}^{T} e^{-\int_{t}^{u} r_{B}(s) d s} d u \\
& r_{G}(t)=\frac{\delta}{\gamma}+\frac{\gamma-1}{\gamma} \tilde{r}(t)+\frac{\gamma-1}{2 \gamma^{2}} \tilde{\Lambda}(t)^{2} \\
& -k\left[\tilde{r}(t)-r_{\omega}(t)+\frac{\tilde{\mu}_{H}(t)}{\gamma}-\frac{\gamma-1}{\gamma}(\chi-m)+\frac{k-1}{2} \sigma_{H}^{2}\right], \\
& r_{F}(t)=\tilde{r}(t)-\mu_{Y}(t)+\sigma_{Y}(t)\left(\psi_{S} \tilde{\lambda}_{S}(t)+\psi_{H} \tilde{\lambda}_{H}(t)+\psi_{Y} \nu_{I}(t)\right) \text {, }
\end{aligned}
$$




$$
r_{B}(t)=r_{\omega}(t)+\varepsilon-\alpha+\chi-m
$$

and $\psi_{S}, \psi_{H}, \psi_{Y}$ are defined by the correlation structure, cf. (60) in Appendix A.4, and $\tilde{\Lambda}(t)^{2}$ is defined by the Sharpe ratios and the correlation structure, cf. (54). Defining disposable wealth as

$$
\widehat{X}_{t}=X_{t}+Y_{t} F_{\theta}(t)-\bar{q}_{t} \chi H_{t} B_{\theta}(t),
$$

the optimal consumption and investment strategy is given by

$$
\begin{aligned}
c_{t} & =b \hat{b} \frac{\left(1+\alpha B_{\theta}(t)\right)^{k}}{G_{\theta}(t)} \widehat{X}_{t}, \\
q_{t} & =\bar{q}_{t}+(1-b) \hat{b} \frac{\left(1+\alpha B_{\theta}(t)\right)^{k-1}}{\chi H_{t} G_{\theta}(t)} \widehat{X}_{t}, \\
\Pi_{S t} & =\frac{\xi_{S}(t)}{\gamma \sigma_{S}} \frac{\widehat{X}_{t}}{X_{t}}-\frac{\sigma_{Y}(t) \psi_{S}}{\sigma_{S}} \frac{Y_{t} F_{\theta}(t)}{X_{t}}, \\
\Pi_{H t} & =\frac{\xi_{H}(t)}{\gamma \sigma_{H}} \frac{\widehat{X}_{t}}{X_{t}}-\frac{\sigma_{Y}(t) \psi_{H}}{\sigma_{H}} \frac{Y_{t} F_{\theta}(t)}{X_{t}}+k \frac{X_{t}}{X_{t}}+\bar{q}_{t} \chi B_{\theta}(t) \frac{H_{t}}{X_{t}}, \\
\Pi_{I t} & =\frac{\xi_{I}(t)}{\gamma} \frac{\widehat{X}_{t}}{X_{t}}-\sigma_{Y}(t) \psi_{Y} \frac{Y_{t} F_{\theta}(t)}{X_{t}}
\end{aligned}
$$

with $\xi_{S}(t), \xi_{H}(t), \xi_{I}(t)$ are defined in terms of the Sharpe ratios and the correlation structure, cf. (57)-(59) in Appendix A.4.

Despite the rich setting, the solution has the same structure as in the special cases in Section 4 and as in simpler models considered in the existing literature. Therefore, the optimal consumption strategy and the optimal stock and house investment strategy have the same interpretation as given after Theorems 1 and 2. The optimal investment in the income derivative consists of a speculative demand and an income-adjustment term. In artificial markets where the income derivative is assumed to be uncorrelated with both stock and house price $\left(\rho_{I S}=\rho_{I H}=0\right.$ and thus $\hat{\rho}_{I H}=0$ and $\left.\hat{\rho}_{I}=1\right)$, we have $\xi_{I}(t)=\nu_{I}(t)$ and $\psi_{Y}=\hat{\rho}_{Y}$ so that the speculative demand is determined by the Sharpe ratio $\nu_{I}(t)$ and the income adjustment term by the unspanned income coefficient $\hat{\rho}_{Y}$.

Note that the elements of $\theta$ that characterize the artificial market enter most terms in the optimal consumption and investment strategy (31)-(35) in this market. Recall that the optimal strategy in any artificial market is infeasible in the true market. Intuitively, we are looking for artificial markets in which the optimal investment in the artificial income derivative is near zero and where the optimal strategies satisfy or are close to satisfying all constraints in the true market. The choice of the functional form of $\nu_{S}, \nu_{H}$, and $\nu_{I}$ 
influences the wealth loss. The affine specification

$$
\nu_{S}(t)=v_{0}^{S}+v_{1}^{S} t, \quad \nu_{H}(t)=v_{0}^{H}+v_{1}^{H} t, \quad \nu_{I}(t)=v_{0}^{I}+v_{1}^{I} t
$$

provides a good trade off between precision and computational complexity. The artificial markets are then characterized by the six constants in these functions plus $\rho_{I S}$ and $\rho_{I H}$.

\subsection{Consumption and investment decisions in the true market}

The optimal strategy in any artificial market is infeasible in the true market, but we transform it into a feasible strategy. In the true market the housing habit buffer at time $t$ is $\bar{q}_{t} \chi H_{t} B(t)$, which is at least as large as the artificial-market buffer $\bar{q}_{t} \chi H_{t} B(t)$ since $r_{\omega}(t) \geq 0$. The agent must ensure that tangible wealth exceeds the true-market buffer, i.e., that $X_{t} \geq \bar{q}_{t} H_{t} B(t)$. When tangible wealth is close to the buffer, current consumption cannot be implicitly financed by future income, which is therefore less valuable. Following BKM, we capture this parsimoniously by reducing the human capital $Y_{t} F_{\theta}(t)$ through a replacement of $F_{\theta}(t)$ by

$$
\widetilde{F}_{\theta}(t)=F_{\theta}(t)\left(1-e^{-\eta\left[X_{t}-\bar{q}_{t} \chi H_{t} B(t)\right]}\right),
$$

where $\eta$ is a positive constant to be determined experimentally. Hence, we replace the disposable wealth in the artificial market by

$$
\widetilde{X}_{t}^{(\theta)}=X_{t}+Y_{t} \widetilde{F}_{\theta}(t)-\bar{q}_{t} \chi H_{t} B(t) .
$$

The adjusted consumption strategy derived from the artificial market $\mathcal{M}_{\theta}$ is thus

$$
c_{t}^{(\theta)}=b \hat{b} \frac{\left(1+\alpha B_{\theta}(t)\right)^{k}}{G_{\theta}(t)} \widetilde{X}_{t}^{(\theta)}, \quad q_{t}^{(\theta)}=\bar{q}_{t}+(1-b) \hat{b} \frac{\left(1+\alpha B_{\theta}(t)\right)^{k-1}}{\chi H_{t} G_{\theta}(t)} \widetilde{X}_{t}^{(\theta)} .
$$

The stock-house investment strategy must respect the constraints (23). Starting from (33)-(34), we adjust the housing habit buffer and the disposable wealth as explained above and take the positive part of the resulting expressions to get

$$
\begin{aligned}
& \widetilde{\Pi}_{S t}^{(\theta)}=\left(\frac{\xi_{S}(t)}{\gamma \sigma_{S}} \frac{\widetilde{X}_{t}^{(\theta)}}{X_{t}}-\frac{\sigma_{Y}(t) \psi_{S}}{\sigma_{S}} \frac{Y_{t} \widetilde{F}_{\theta}(t)}{X_{t}}\right)^{+}, \\
& \widetilde{\Pi}_{H t}^{(\theta)}=\left(\frac{\xi_{H}(t)}{\gamma \sigma_{H}} \frac{\widetilde{X}_{t}^{(\theta)}}{X_{t}}-\frac{\sigma_{Y}(t) \psi_{H}}{\sigma_{H}} \frac{Y_{t} \widetilde{F}_{\theta}(t)}{X_{t}}+k \frac{\widetilde{X}_{t}^{(\theta)}}{X_{t}}+\bar{q}_{t} \chi B(t) \frac{H_{t}}{X_{t}}\right)^{+},
\end{aligned}
$$




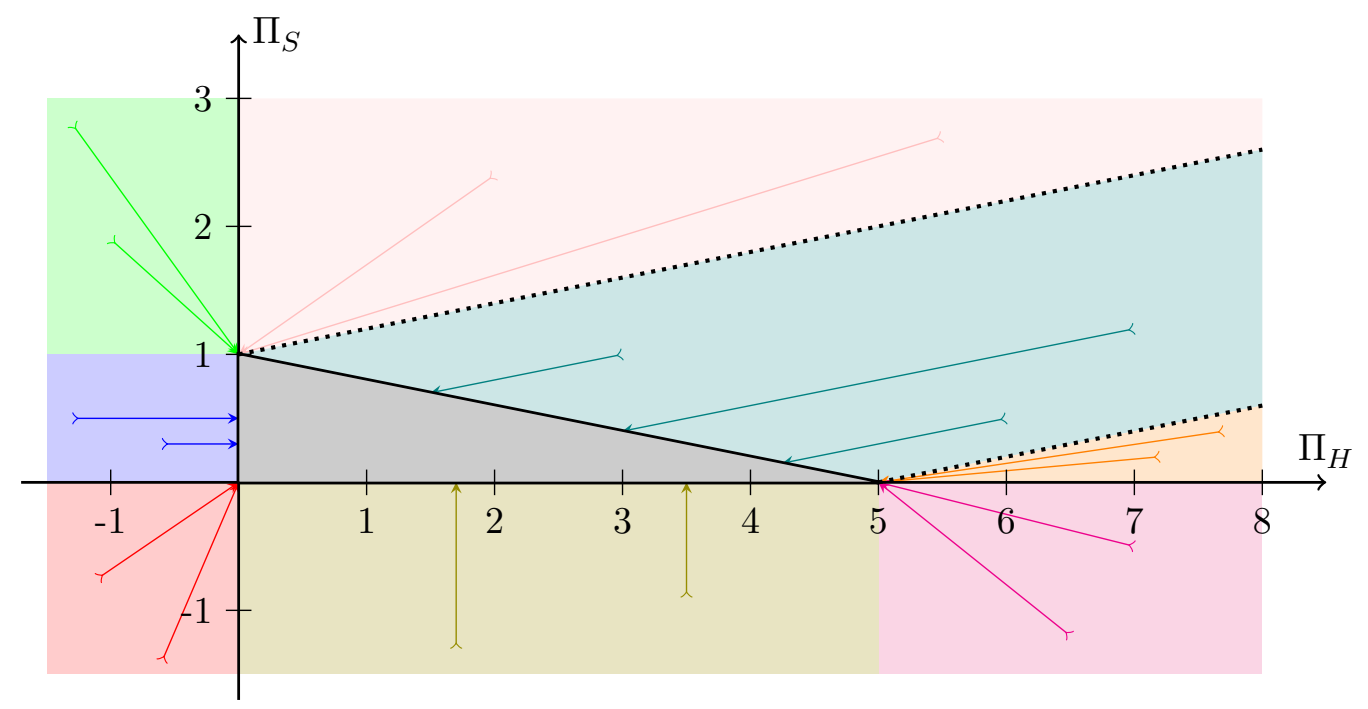

Figure 6: The transformation of portfolio weights. The gray triangle is the feasible region. We assume $\kappa=0.2$. The transformation depends on which colored area the artificial market portfolio is located in. The arrows show examples of the transformation.

where $x^{+}=\max (x, 0)$. If $\widetilde{\Pi}_{S t}^{(\theta)}+\kappa \widetilde{\Pi}_{H t}^{(\theta)} \leq 1$, we just use the strategy

$$
\Pi_{S t}^{(\theta)}=\widetilde{\Pi}_{S t}^{(\theta)}, \quad \Pi_{H t}^{(\theta)}=\widetilde{\Pi}_{H t}^{(\theta)} .
$$

In other cases we trim the portfolio weights as explained in Cvitanić and Karatzas (1992, Example 14.9) to

$$
\left(\Pi_{S t}^{(\theta)}, \Pi_{H t}^{(\theta)}\right)= \begin{cases}(1,0), & \text { if } \widetilde{\Pi}_{S t}^{(\theta)}-\kappa \widetilde{\Pi}_{H t}^{(\theta)} \geq 1, \\ \left(0, \frac{1}{\kappa}\right), & \text { if } \widetilde{\Pi}_{S t}^{(\theta)}-\kappa \widetilde{\Pi}_{H t}^{(\theta)} \leq-1, \\ \left(\frac{1+\widetilde{\Pi}_{S t}^{(\theta)}-\kappa \widetilde{\Pi}_{H t}^{(\theta)}}{2}, \frac{1-\widetilde{\Pi}_{S t}^{(\theta)}+\kappa \widetilde{\Pi}_{H t}^{(\theta)}}{2 \kappa}\right), & \text { if }\left|\widetilde{\Pi}_{S t}^{(\theta)}-\kappa \widetilde{\Pi}_{H t}^{(\theta)}\right|<1 .\end{cases}
$$

The transformation of the portfolio weights are illustrated in Figure 6 .

For each $\theta \in \Theta$, the transformed strategy $a_{\theta}=\left(c^{(\theta)}, q^{(\theta)}, \Pi_{S}^{(\theta)}, \Pi_{H}^{(\theta)}\right)$ is a feasible strategy in the true market and generates an expected utility $J\left(t, x, y, h, \bar{q} ; a_{\theta}\right)$ that we can estimate via Monte Carlo simulation. The performance of the strategy $a_{\theta}$ can be quantified by the wealth equivalent loss $L_{\theta}$ relative to the upper bound on the expected utility, $\bar{J}$, defined in (29). The loss is implicitly defined by

$$
J\left(t, x, y, h, \bar{q} ; a_{\theta}\right)=\bar{J}(t, x[1-L], y[1-L], h, \bar{q}),
$$


so $L$ is an upper bound on the relative reduction in initial wealth $x$ and income $y$ the agent would accept to get access to the truly optimal (but unkonwn) strategy. Note that $L$ has to be determined by a numerical search routine. The upper utility bound $\bar{J}$ is the value function in some artificial market $\bar{\theta}$ that we find by a numerical minimization of (30). The left-hand side in (36) is determined by Monte Carlo simulation so to avoid a simulation bias we also compute the right-hand side by simulation of the optimal strategy in the market corresponding to the previously determined $\bar{\theta}$.

\section{Results with benchmark parameter values}

Table 1 summarizes the parameters we use as a benchmark. Monetary quantities such as initial wealth and income are measured in thousands of USD. For example, the initial house price $H_{0}=0.25$ corresponds to 250 USD per square foot, so 250,000 USD for a home of 1000 square feet of average quality and location.

The parameter values for stock prices, house prices, and labor income are slightly rounded estimates based on the following procedure. The estimates are obtained by first using U.S. data on the stock market index, the national house price index, and aggregate labor income, and subsequently house price volatility and income volatility are adjusted to be more representative of individual house prices and labor income. We use quarterly U.S. data for stock prices, house prices and aggregate labor income starting in 1953q1 and ending in 2010q4. For stocks, we use quarterly returns on the CRSP value-weighted market portfolio inclusive of the NYSE, AMEX, and NASDAQ markets. The risk-free rate is the three month Treasury bill yield from the Risk Free File on CRSP Bond tape. The house price is represented by the national Case-Shiller home price index with data taken from Robert Shiller's homepage. ${ }^{16}$ From the National Income and Product Accounts (NIPA) tables published by the Bureau of Economic Analysis of the U.S. Department of Commerce, we obtain quarterly U.S. data for aggregated disposable personal income. We divide the disposable income by the population size reported in the NIPA table to compute the disposable labor income per capita. To compute real values, all time series are deflated using the consumer price index (CPI) taken from CRSP. Figure 6 depicts the time series of the stock price index, per capita labor income, and the national home price index.The stock price parameters are quite standard. Note that the estimated expected growth rate of (real) house price is only $r+\mu_{H}=0.04 \%$.

We increase the volatility of the house series to $12 \%$, which is similar to the existing literature, e.g., Flavin and Yamashita (2002) and Yao and Zhang (2005). Furthermore, we adjust the income volatility to $\sigma_{Y}=0.10$. This is in line with Cocco, Gomes, and Maen-

\footnotetext{
${ }^{16}$ http://www.econ,yale.edu/ shiller/data.htm
} 


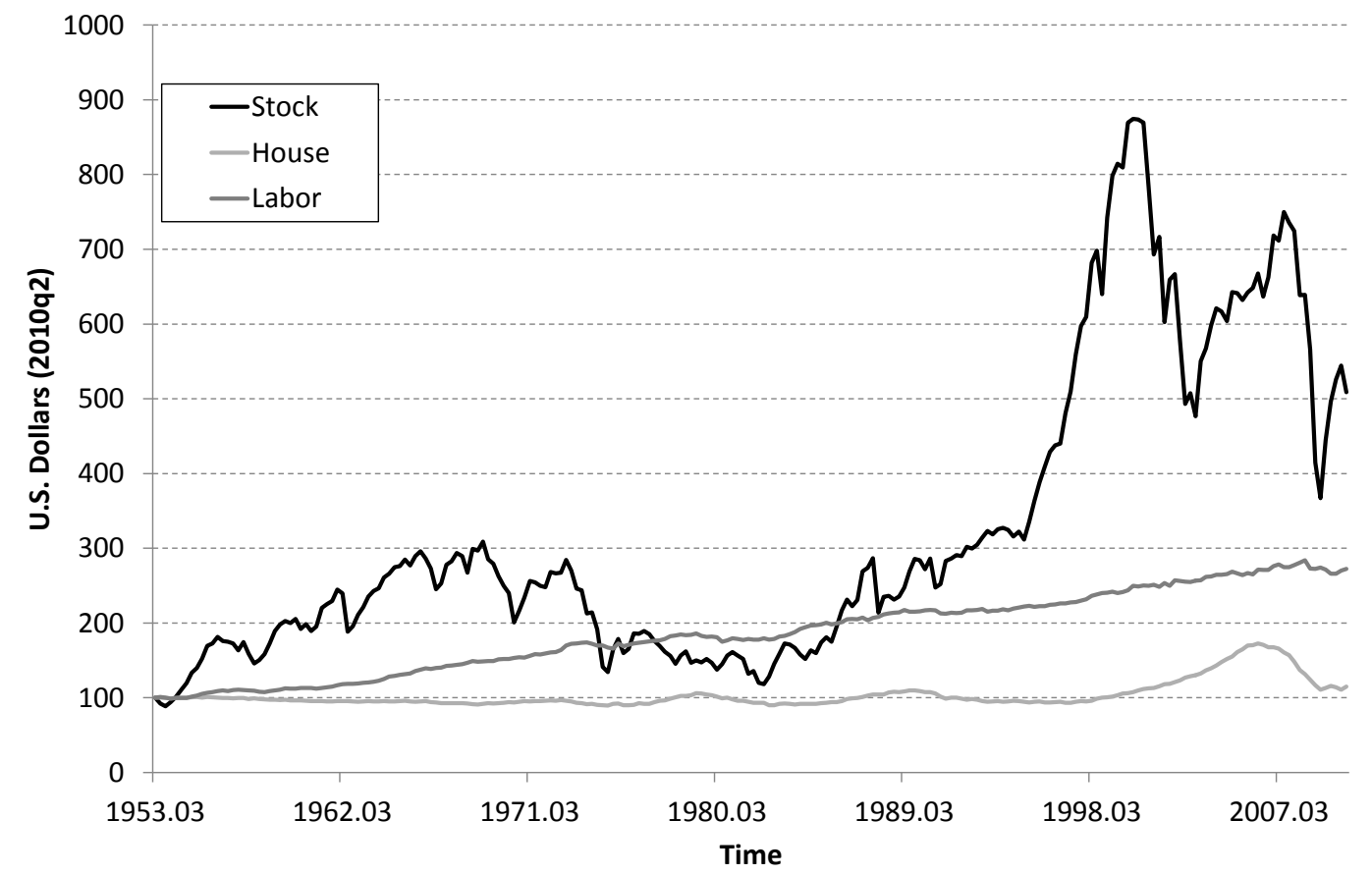

Figure 7: Historical evolution of stock market, house prices, and income per capita in the U.S. The stock market is represented by the S\&P composite stock price index without dividends. The house prices uses the national Case-Shiller home price index. Income is disposable labor income per capita from NIPA. All time series are standardized to 100 in $1953 \mathrm{q} 2$ and inflation-adjusted to $2010 \mathrm{q} 2$ dollars using CPI. 


\begin{tabular}{llr}
\hline Parameter & Description & Value \\
\hline$\delta$ & time preference rate & 0.05 \\
$\gamma$ & relative risk aversion & 3 \\
$b$ & perishable/housing utility weight & 0.69 \\
$w$ & weight of bequest & 0 \\
$\alpha$ & habit scaling parameter & 0.8 \\
$\varepsilon$ & habit persistence parameter & 0.9 \\
$\bar{q}_{0}$ & initial housing habit level & 150 \\
$T$ & remaining life time & 50 \\
$\tilde{T}$ & retirement date & 30 \\
\hline$X_{0}$ & initial financial wealth & 20 \\
$r$ & risk-free rate & 0.01 \\
$\mu_{S}$ & excess expected stock return & 0.04 \\
$\sigma_{S}$ & stock volatility & 0.17 \\
$h_{0}$ & initial unit house price & 0.25 \\
$\mu_{H}$ & excess expected house price growth & -0.0096 \\
$\sigma_{H}$ & house volatility & 0.12 \\
$\chi$ & rental rate & 0.06 \\
$m$ & maintenance cost & 0.025 \\
$\kappa$ & collateral parameter & 0.5 \\
\hline$y_{0}$ & initial income per year & 20 \\
$\mu_{Y}$ & expected income growth & 0.01 \\
$\sigma_{Y}$ & income volatility & 0.1 \\
$\Upsilon$ & replacement ratio & 0.6 \\
\hline$\rho_{H S}$ & house-stock correlation & 0.25 \\
$\rho_{Y S}$ & income-stock correlation & 0.22 \\
$\rho_{Y H}$ & income-house correlation & 0.16 \\
\hline & &
\end{tabular}

Table 1: Benchmark parameter values.

hout (2005), among others. We allow income volatility (but a zero drift) in retirement as motivated earlier, and assume that the same value applies there as before retirement. The values of risk aversion and other agent-specific parameters are standard in the literature. At this point, we assume a constant income drift of $1 \%$ throughout the working phase.

The results presented in the following are averages based on simulations of 10000 paths. The strategy are updated 50 times per year. The upper bound in the artificially completed market is computed by Monte Carlo simulation as well to avoid any simulation bias. With our benchmark parameters, the loss bound is $1.6 \%$, so by applying the consumptioninvestment strategy suggested by our method instead of the truly optimal and unknown strategy, the agent suffers a utility loss corresponding to at most $1.6 \%$ of her wealth. Recall that in the experiments reported by BKM, the actual loss is much smaller than the loss bound. 
Figure 8 illustrates various aspects of the optimal investments over the life cycle. Initially the agent borrows 20 and invests that amount plus her initial wealth of 20 in housing units, and nothing in stocks. This corresponds to a portfolio weight of 2 for housing, 0 for stocks, and -1 for the risk-free asset. If we assume the agent is initially 30 years, she does not invest in stocks at all until age 37-38, after which the stock investments gradually increase until retirement, and then the stock investments decrease towards zero through retirement. Note that this is obtained for a risk aversion coefficient as low as three. This pattern is in stark contrast to life-cycle models without housing and housing habits. For example, Cocco, Gomes, and Maenhout (2005) find that even with a risk aversion of 10, the agent typically starts out investing $100 \%$ of wealth in the stock market and after some years, the stock weight is gradually reduced to around $50 \%$ around retirement. Our model thus provides an explanation for the observed non-participation in stock markets of many young individuals without referring to (more or less randomly chosen) stock market entry costs. The stock investment peaks at around 70 thousand USD around retirement.

The portfolio is dominated by housing investments, partly funded by collateralized borrowing, throughout life. Housing investments are modestly attractive from a speculative view, but offer access to borrowing, hedge against increasing housing consumption prices, and ensures that future housing consumption habits can be covered. The housing investment peaks at around 200 thousand dollars at retirement.

The lower-left panel confirms that the housing habit induces a larger housing investment throughout life. The larger holdings of housing units due to the habit imply a larger financial wealth as seen in the upper-right panel. This causes a larger stock investment as well in the presence of the habit. The portfolio weight of the stock shown in the middle-right panel is roughly the same with and without the habit up to retirement, but significantly lower with the habit in retirement.

Figure 9 depicts the optimal consumption over the life cycle. The graphs are similar to those presented in the deterministic model in Section 4.1. The left panel shows that perishable consumption in the no-habit model is increasing through life, although relatively flat in retirement. With the housing habit, perishable consumption is hump shaped with the peak around retirement for this parameter set. Note that Cocco, Gomes, and Maenhout (2005), among others, also report a consumption hump around retirement and explain it by a mix of mortality risk, borrowing constraints, and a hump-shaped income profile. We obtain the consumption hump without mortality risk or a hump-shaped income profile, and with much less tight borrowing constraints due to the access to borrowing collateralized by home ownership. As illustrated in Section 4.1, the peak age can occur earlier for stronger habits, and with the addition of mortality risk and bequest. The right panel confirms that the housing habits produce a realistic life-cycle pattern in the housing 

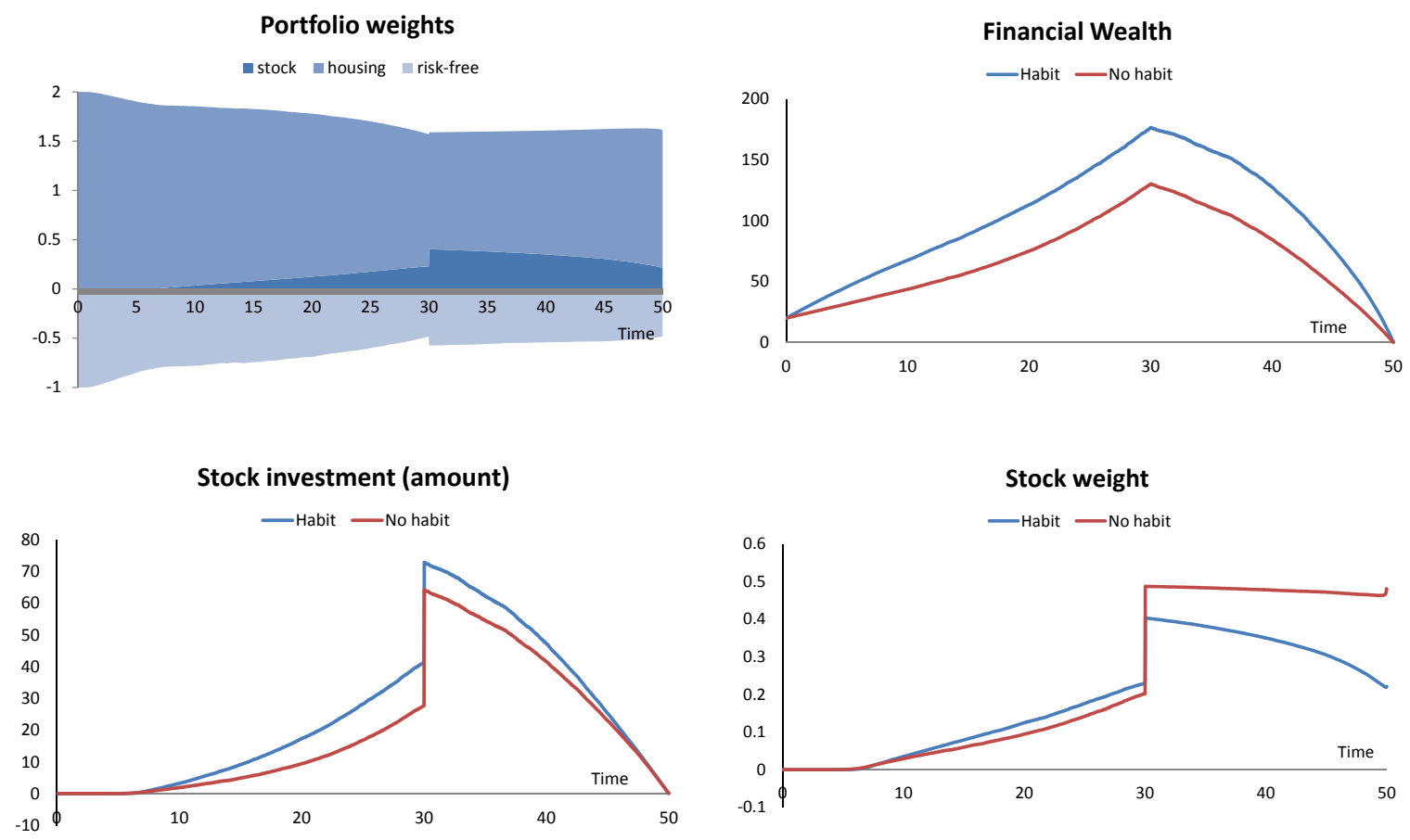

Housing Investment (amount)

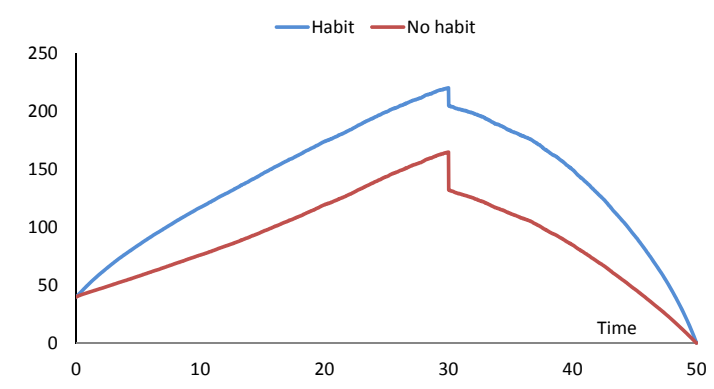

Risk-free investment

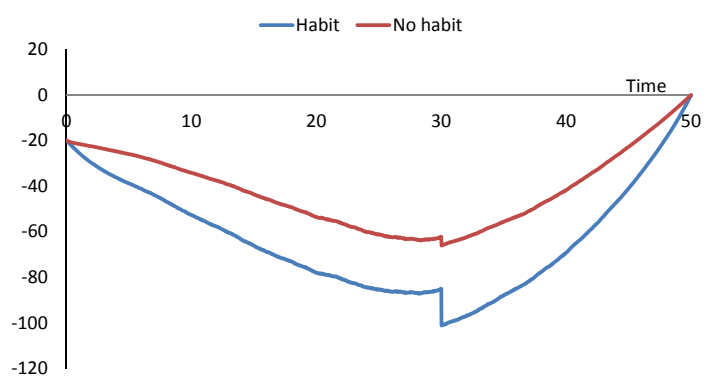

Figure 8: Investments over the life cycle in the full model. 

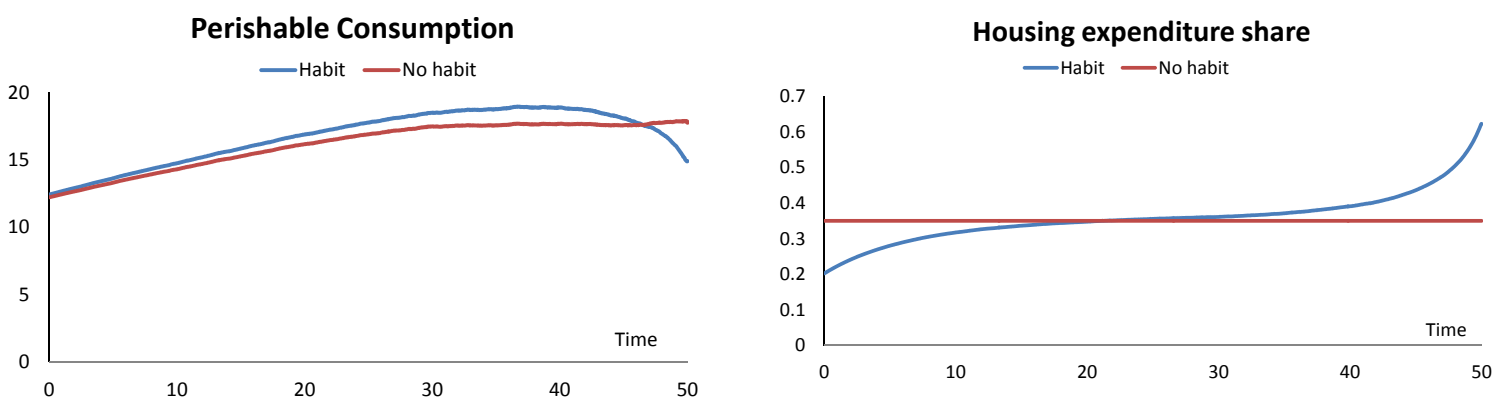

Figure 9: Consumption over the life cycle in the full model.

expenditure share, unlike the constant share implied by the no-habit model.

Figure 10 compares optimal housing consumption and optimal housing investment over the life cycle. Without housing habits, the optimal housing consumption exceeds the optimal investment position at all ages. With the habit, optimal housing consumption is first above the investment position, then below, and then above again in retirement. This might be interpreted such that the agent prefers to rent her home early in life, then shifts to home ownership, and later shifts back to renting. Such a pattern is often observed in real life.

\section{Some model extensions and robustness checks}

Figure 11 illustrates the importance of the habit strength for our results. Recall that the habit dynamics involve the two parameters $\varepsilon$ and $\alpha$, and that the difference $\varepsilon-\alpha$ represents the strength of the habit. If $\varepsilon-\alpha$ is small, the habit only declines very slowly even with minimum housing consumption and therefore restricts the agent more. The figure fixes $\varepsilon=0.9$ and considers $\alpha=0.7$ (weak habit) and $\alpha=0.88$ (strong habit). The upper-left graph confirms that the agent must save more if the habit is strong, and the upper-right graph shows that the extra saving is implemented mainly by increasing the housing investment position. Still the amount invested in the stock is also increased, especially in the years leading up to retirement, as shown in the panels in the middle. The portfolio share of the stock is almost independent of the habit strength before retirement, but in retirement the share is much higher for the weak habit than for the strong habit. The lower-left panel reveals that the life-cycle pattern in perishable consumption is almost the same for the two habit strengths, but with the strong habit the pattern is more curved and the peak appears earlier in life, as we also saw in the deterministic model of Section 4.1. The housing expenditure share in the lower-right panel is increasing through life, but with a relatively weak habit the share is almost constant for a long mid-life period. 


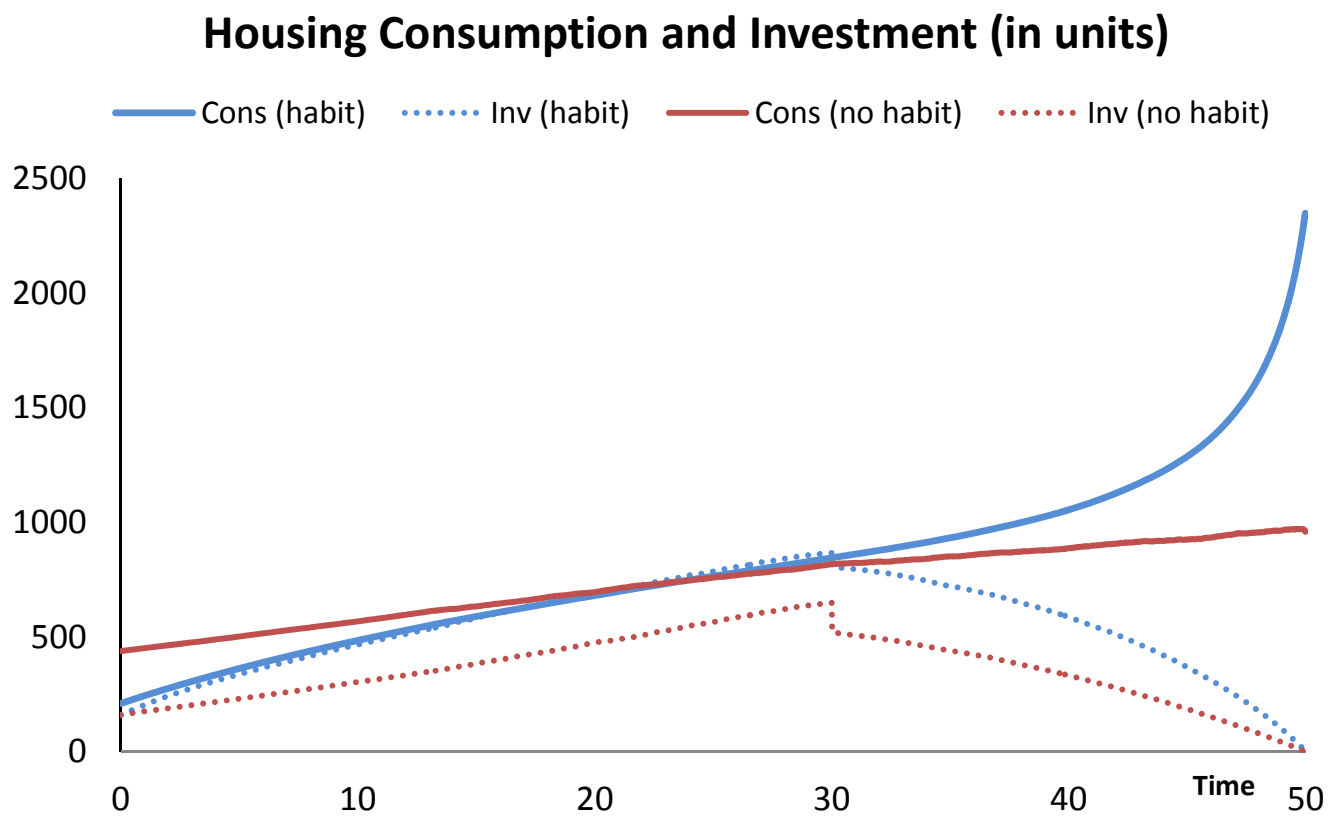

Figure 10: Housing consumption and investment over the life cycle in the full model.

Figure 12 illustrates the role of the parameter $b$, which is the preference weight of perishable consumption, cf. (2), and in the unconstrained no-habit case $b$ is also the expenditure share of perishable consumption. As we can see in the upper-right panel, also in the full model with housing habits $b$ has the expected effect on the housing expenditure share, which is roughly $1-b$ in mid life, lower early in life, and higher late in life. With low $b$ and thus a high preference for housing consumption, the agent builds a higher financial wealth (upper-left panel), and this comes from a higher housing investment (lower-left panel), whereas the stock investment is a bit smaller. With a high desired future housing consumption, the agent hedges the costs thereof by investing in more housing units in line with the argument of Sinai and Souleles (2005). As we can see by comparing the solid lines (habit) and the dotted lines (no habit) in the lower-left panel, the effect is more pronounced with housing habits due to the associated housing habit buffer.

In the previous section we assumed a constant expected income growth rate in the working phase, but it is well known that income on average grows faster for young adults than for more mature adults. We follow Cocco, Gomes, and Maenhout (2005) and use their estimated income profiles for three different educational levels labeled 'no high school', 'high school', and 'college.' Their estimated profiles determines $\mu_{Y}(t)$ in our model. In addition, the initial income is set to 22 for the agent with no high school, 27 with high school, and 37 with a college degree. These values correspond to the average income of a 


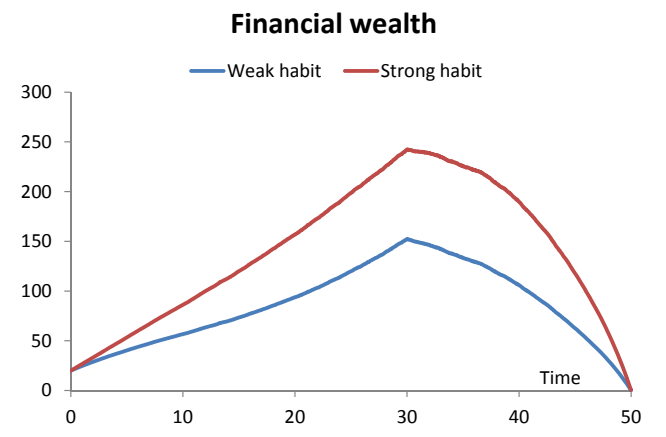

Stock investment (amount)

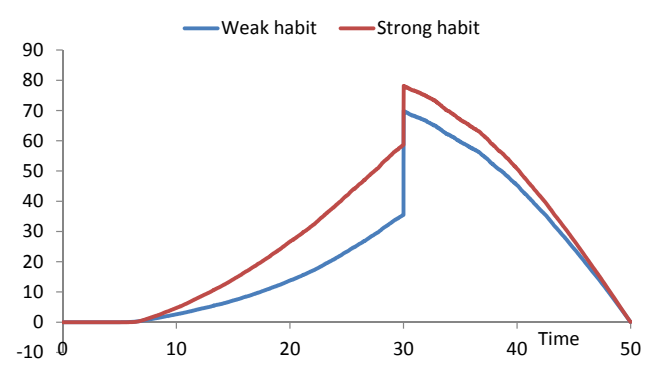

Perishable consumption

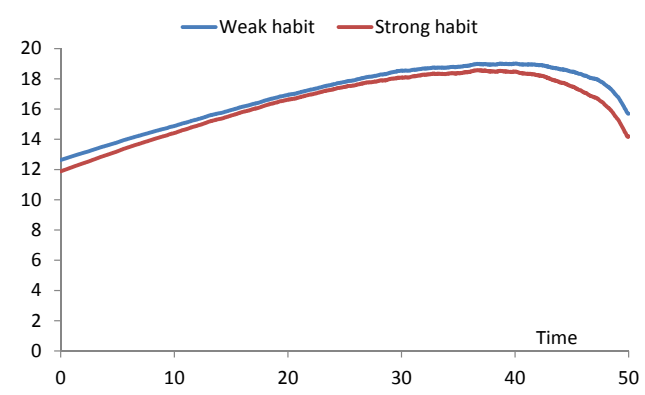

Housing investment (amount)

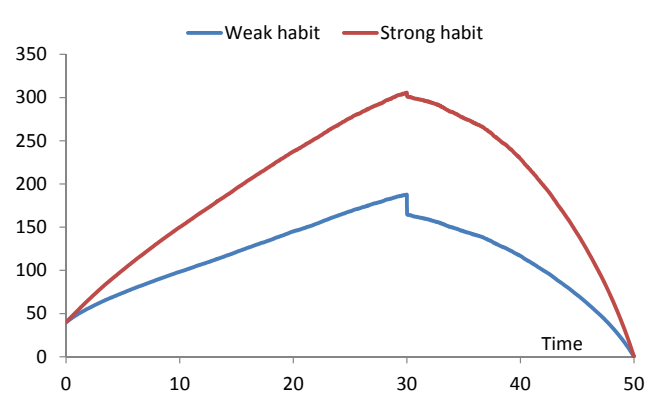

Stock investment (weight)

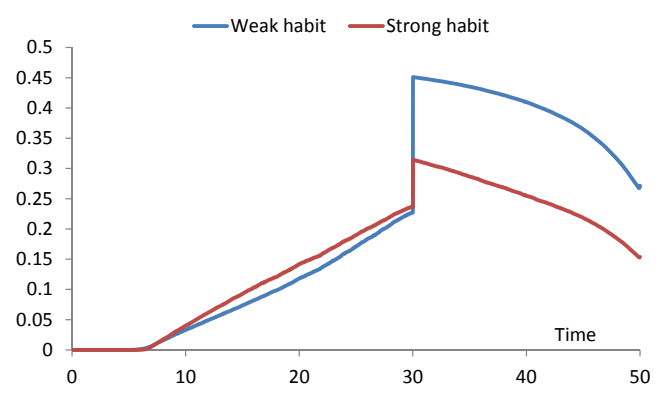

Housing expenditure share

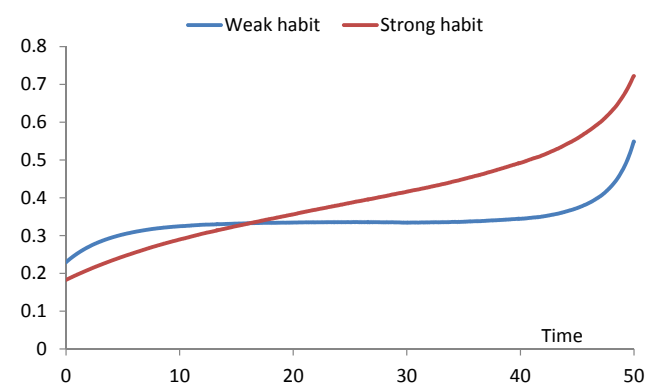

Figure 11: Consumption and investments for different habit strengths. Here weak habit means $\alpha=0.7$ and strong habit means $\alpha=0.88$. 

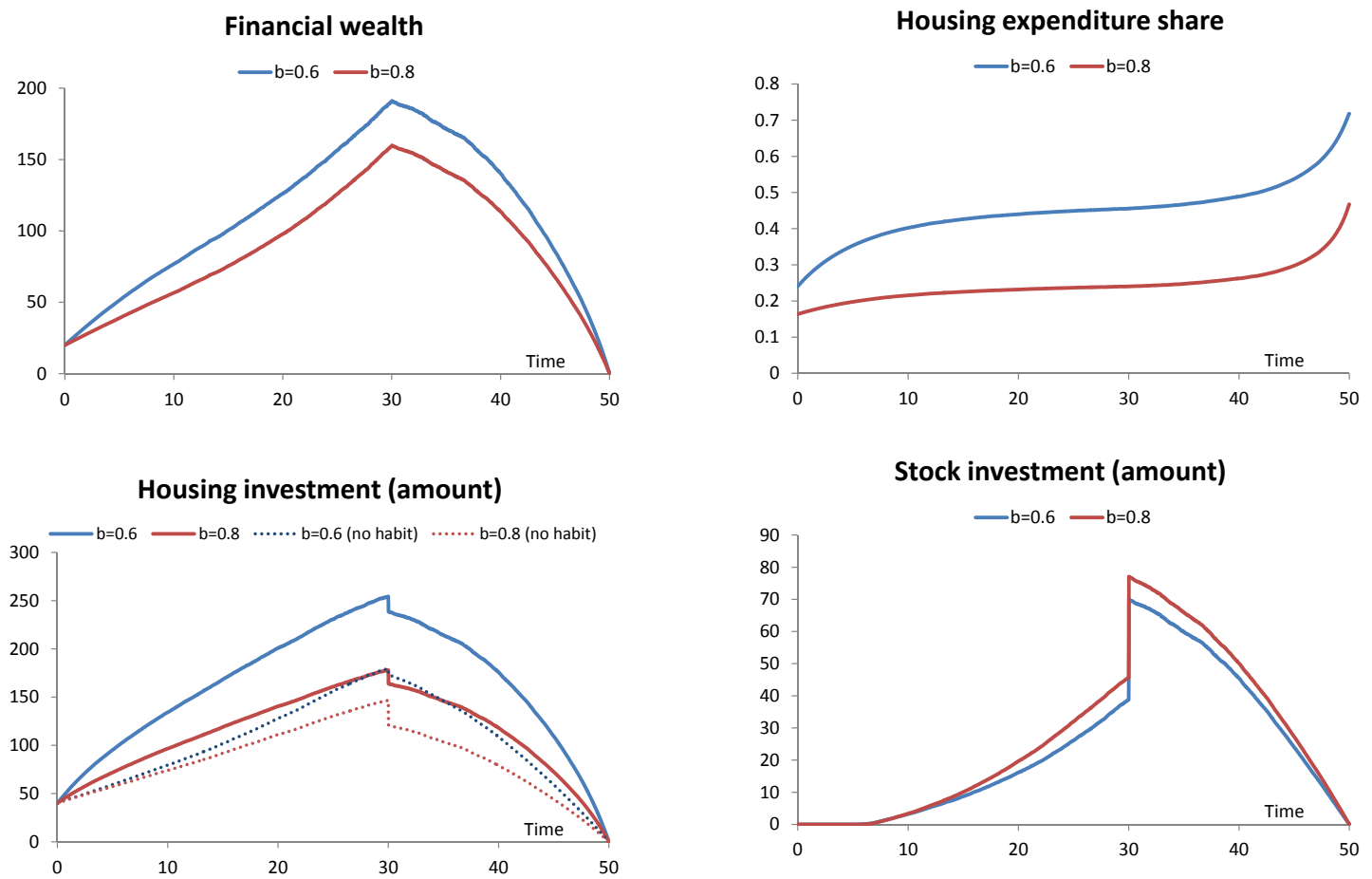

Figure 12: Consumption and investments for different values of $b$.

35-year old in the different educational groups, according to Cocco, Gomes, and Maenhout (2005). The other parameters are unchanged compared to the benchmark case. The left panel of Figure 13 shows the optimal perishable consumption over life. The overall shape of the curve is the same for all three educational levels, but of course the level of consumption increases with income and thus education.

\section{Conclusion}

We have solved for and investigated the optimal life-cycle consumption and investment decisions in a rich model with many realistic features. In particular, we include preferences for both perishable consumption and consumption of housing services with habit formation for housing. We have provided closed-form solutions for simplified settings to build intuition and used an innovative numerical solution method for the full model with portfolio constraints and unspanned income risk.

Our results show that the model can generate various stylized facts that seem puzzling through the lens of standard life-cycle models. First, stock investments are low or zero for many young agents and then gradually increasing over life. Housing investments crowd out stock investments, in particular for young agents, because housing investments (i) provide a hedge against increases in the price of housing consumption, (ii) ensure that the agent can 

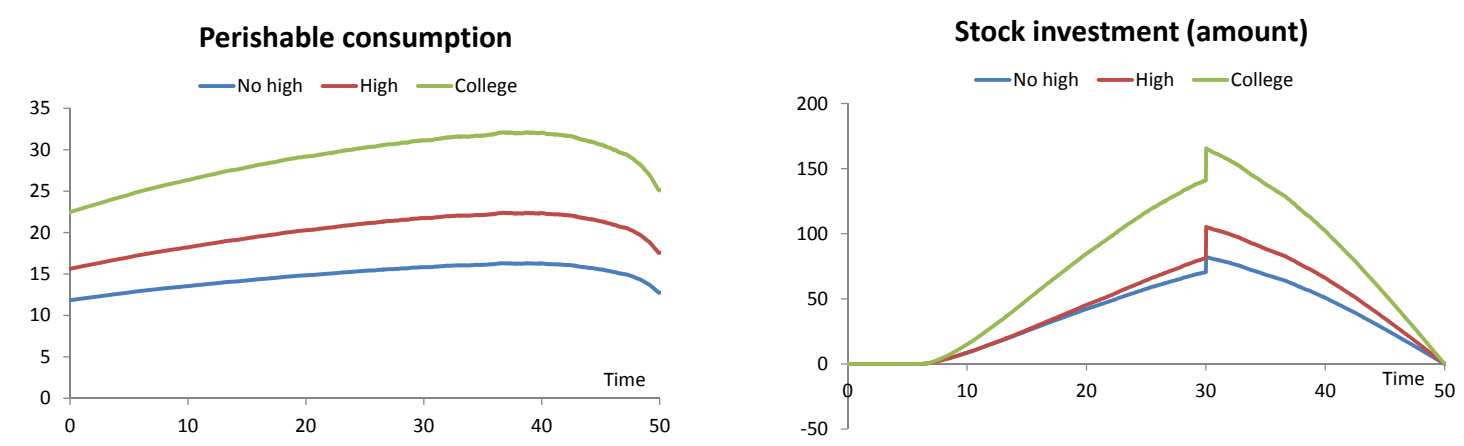

Figure 13: Consumption and stock investments for different educational levels.

obtain the future minimum housing consumption generated by her habits, and (iii) are not a bad investment from a purely speculative view. Secondly, our model generates an ageand wealth-dependent housing expenditure share instead of the counter-factually constant share found in standard models. Thirdly, in our model perishable consumption is much more sensitive to wealth and income shocks than housing consumption, in particular for young agents. Finally, the housing habit helps in explaining the hump-shaped life-cycle pattern in non-housing consumption. 


\section{A Proofs}

\section{A.1 Proof of Theorem 1}

The Bellman equation is

$$
0=\mathcal{L}_{1} J+\mathcal{L}_{2} J
$$

where

$$
\begin{aligned}
& \mathcal{L}_{1} J=\sup _{c, q}\left\{-c J_{x}-(q-\bar{q}) h \chi J_{x}+\frac{1}{1-\gamma} c^{b(1-\gamma)}(q-\bar{q})^{(1-b)(1-\gamma)}+\alpha(q-\bar{q}) J_{\bar{q}}\right\} \\
& \mathcal{L}_{2} J=J_{t}+r x J_{x}+y J_{x}+\left(r+\mu_{H}\right) h J_{h}+\mu_{Y} y J_{y}-\bar{q} h \chi J_{x}+(\alpha-\varepsilon) \bar{q} J_{\bar{q}}-\delta J
\end{aligned}
$$

The first-order conditions imply

$$
\begin{aligned}
b c^{b(1-\gamma)-1}(q-\bar{q})^{(1-b)(1-\gamma)} & =J_{x}, \\
(1-b) c^{b(1-\gamma)}(q-\bar{q})^{(1-b)(1-\gamma)-1} & =\chi h J_{x}-\alpha J_{\bar{q}},
\end{aligned}
$$

and by dividing one by the other we see that

$$
c=\frac{b}{1-b}(q-\bar{q})\left(\chi h-\alpha \frac{J_{\bar{q}}}{J_{x}}\right) .
$$

Substituting this relation back into one of the first-order conditions we obtain

$$
c=b^{\frac{1}{\gamma}}\left(\frac{b}{1-b}\right)^{\frac{(1-b)(\gamma-1)}{\gamma}}\left(\chi h-\alpha \frac{J_{\bar{q}}}{J_{x}}\right)^{\frac{(1-b)(\gamma-1)}{\gamma}} J_{x}^{-\frac{1}{\gamma}}=b \hat{b}\left(\chi h-\alpha \frac{J_{\bar{q}}}{J_{x}}\right)^{k} J_{x}^{-\frac{1}{\gamma}},
$$

and subsequently we get

$$
q=\bar{q}+(1-b) \hat{b}\left(\chi h-\alpha \frac{J_{\bar{q}}}{J_{x}}\right)^{k-1} J_{x}^{-\frac{1}{\gamma}} .
$$

After substitution of the optimal controls it follows that

$$
\mathcal{L}_{1} J=\frac{\gamma}{1-\gamma} \hat{b}\left(\chi h-\alpha \frac{J_{\bar{q}}}{J_{x}}\right)^{k} J_{x}^{\frac{\gamma-1}{\gamma}} .
$$

Conjecture a value function of the form (5). With $\hat{x}=x+y F(t)-\bar{q} \chi h B(t)$, the relevant derivatives are

$$
\begin{gathered}
J_{t}=\hat{x}^{-\gamma}(\chi h)^{\gamma k} G^{\gamma-1}\left[\frac{\gamma}{1-\gamma} \hat{x} G^{\prime}+G y F^{\prime}-\bar{q} \chi h B^{\prime} G\right], \quad J_{x}=\hat{x}^{-\gamma} G^{\gamma}(\chi h)^{\gamma k}, \\
J_{\bar{q}}=-(\chi h)^{1+\gamma k} B \hat{x}^{-\gamma} G^{\gamma}, \quad J_{y}=\hat{x}^{-\gamma}(\chi h)^{\gamma k} G^{\gamma} F, \quad J_{h}=\hat{x}^{-\gamma}(\chi h)^{\gamma k} h^{-1} G^{\gamma}\left[\frac{k \gamma}{1-\gamma} \hat{x}-\bar{q} \chi h B\right],
\end{gathered}
$$


from which (9) follows. Note that $J_{\bar{q}} / J_{x}=-h \chi B(t)$. Now substitute the derivatives into (38) and (39), replace $r x$ by $r \hat{x}-r y F+r \bar{q} \chi h B$, and reduce to get

$$
\begin{aligned}
\mathcal{L}_{1} J=\hat{x}^{1-\gamma}(\chi h)^{\gamma k} G^{\gamma-1} & \frac{\gamma}{1-\gamma} \hat{b}(1+\alpha B(t))^{k} \\
\mathcal{L}_{2} J=\hat{x}^{-\gamma}(\chi h)^{\gamma k} G^{\gamma-1} & \left\{\frac{\gamma}{1-\gamma} \hat{x}\left[G^{\prime}(t)-\left(\frac{\delta}{\gamma}+\frac{\gamma-1}{\gamma} r-\left(r+\mu_{H}\right) k\right) G(t)\right]\right. \\
& +G(t) y\left[F^{\prime}(t)+\left(\mu_{Y}(t)-r\right) F(t)+1\right] \\
& \left.-\bar{q} h G(t)\left[B^{\prime}(t)-\left(\varepsilon-\alpha-\mu_{H}\right) B(t)+1\right]\right\} .
\end{aligned}
$$

After adding up and dividing by $\hat{x}^{-\gamma}(\chi h)^{\gamma k} G^{\gamma-1}$, each remaining term is either a multiple of $\hat{x}$ or does not involve $\hat{x}$. Collecting the terms involving $\hat{x}$, we conclude that $G$ must satisfy

$$
0=G^{\prime}(t)-r_{G} G(t)+\hat{b}(1+\alpha B(t))^{k}
$$

which with $G(T)=0$ (due to no bequests) has the solution (7). The terms not involving $\hat{x}$ add up to zero if

$$
0=F^{\prime}(t)-\left(r-\mu_{Y}(t)\right) F(t)+1, \quad 0=B^{\prime}(t)-r_{B} B(t)+1 .
$$

With $F(T)=B(T)=0$, the solutions are as stated in (8) and (6).

Next we derive the dynamics of perishable consumption. By applying (43) as well as the optimal controls (9), we obtain

$$
\begin{aligned}
d \widehat{X}_{t} & =d X_{t}+F(t) d Y_{t}+Y_{t} F^{\prime}(t) d t-\chi H_{t} B(t) d \bar{q}_{t}-\bar{q}_{t} \chi B(t) d H_{t}-\bar{q}_{t} \chi H_{t} B^{\prime}(t) d t \\
& =r \widehat{X}_{t} d t-c_{t} d t-\left(q_{t}-\bar{q}_{t}\right) \chi H_{t}(1+\alpha B(t)) d t \\
& =\widehat{X}_{t}\left(r-\frac{\hat{b}(1+\alpha B(t))^{k}}{G(t)}\right) d t .
\end{aligned}
$$

From (9), it follows that

$$
\begin{aligned}
d c_{t} & =c_{t} \frac{d \widehat{X}_{t}}{\widehat{X}_{t}}+c_{t}\left(\frac{k \alpha B^{\prime}(t)}{1+\alpha B(t)}-\frac{G^{\prime}(t)}{G(t)}\right) d t \\
& =c_{t}\left(r-r_{G}+\frac{k \alpha B^{\prime}(t)}{1+\alpha B(t)}\right) d t \\
& =c_{t}\left(\frac{r-\delta}{\gamma}+k\left[r+\mu_{H}+\frac{\alpha B^{\prime}(t)}{1+\alpha B(t)}\right]\right) d t
\end{aligned}
$$

where we have used (42). Note that $B^{\prime}(t)=-e^{-r_{B}(T-t)}<0$. The growth rate $\mu_{c}(t)$ is 
initially positive provided

$$
\frac{r-\delta}{\gamma} \geq k\left[-\frac{\alpha B^{\prime}(0)}{1+\alpha B(0)}-\left(r+\mu_{H}\right)\right]=k\left[\frac{\alpha r_{B}}{\left(\alpha+r_{B}\right) e^{r_{B} T}-\alpha}-\left(r+\mu_{H}\right)\right] .
$$

Since $B^{\prime \prime}(t)=r_{B} B^{\prime}(t)$, we get

$$
\mu_{c}^{\prime}(t)=k \alpha \frac{B^{\prime \prime}(t)[1+\alpha B(t)]-\alpha\left[B^{\prime}(t)\right]^{2}}{(1+\alpha B(t))^{2}}=k \alpha \frac{\left(r_{B}+\alpha\right) B^{\prime}(t)}{(1+\alpha B(t))^{2}}=k \alpha \frac{\left(\varepsilon-\mu_{H}\right) B^{\prime}(t)}{(1+\alpha B(t))^{2}},
$$

which is negative if $\mu_{H}<\varepsilon$. Hence the consumption growth rate can eventually turn negative and will do so provided

$$
\frac{r-\delta}{\gamma} \leq-k\left(r+\mu_{H}+\frac{\alpha B^{\prime}(T)}{1+\alpha B(T)}\right)=k\left(\alpha-\left[r+\mu_{H}\right]\right) .
$$

This completes the proof.

\section{A.2 Proof of Theorem 2}

It is useful to introduce some vector-matrix notation. Let

$$
\begin{gathered}
\Pi=\left(\begin{array}{c}
\Pi_{S} \\
\Pi_{H}
\end{array}\right), \quad \mu=\left(\begin{array}{c}
\mu_{S} \\
\mu_{H}+\chi-m
\end{array}\right), \quad \Sigma=\left(\begin{array}{cc}
\sigma_{S} & 0 \\
\sigma_{H} \rho_{H S} & \sigma_{H} \sqrt{1-\rho_{H S}^{2}}
\end{array}\right), \\
\vec{\rho}_{H}=\left(\begin{array}{c}
\rho_{H S} \\
\sqrt{1-\rho_{H S}^{2}}
\end{array}\right), \quad \vec{\rho}_{Y}=\left(\begin{array}{c}
\rho_{Y S} \\
\sqrt{1-\rho_{Y S}^{2}}
\end{array}\right)
\end{gathered}
$$

For later use, note that

$$
\begin{aligned}
\left(\Sigma \Sigma^{\top}\right)^{-1} \mu & =\frac{1}{1-\rho_{H S}^{2}}\left(\begin{array}{c}
\frac{\lambda_{S}-\rho_{H S} \lambda_{H}}{\sigma_{S}} \\
\frac{\lambda_{H}-\rho_{H S} \lambda_{S}}{\sigma_{H}}
\end{array}\right), \\
\left(\Sigma^{\top}\right)^{-1} \vec{\rho}_{H} & =\left(\begin{array}{c}
0 \\
\frac{1}{\sigma_{H}}
\end{array}\right), \\
\left(\Sigma^{\top}\right)^{-1} \vec{\rho}_{Y} & =\left(\begin{array}{c}
\frac{\rho_{Y S}-\rho_{H S} \tilde{\rho}_{H Y}}{\sigma_{S}} \\
\frac{\tilde{\rho}_{H Y}}{\sigma_{H}}
\end{array}\right), \\
\Lambda^{2} \equiv \mu^{\top}\left(\Sigma \Sigma^{\top}\right)^{-1} \mu & =\lambda_{S}^{2}+\frac{1}{1-\rho_{H S}^{2}}\left(\lambda_{H}-\rho_{H S} \lambda_{S}\right)^{2}, \\
\mu^{\top}\left(\Sigma^{\top}\right)^{-1} \vec{\rho}_{H} & =\lambda_{H}, \\
\mu^{\top}\left(\Sigma^{\top}\right)^{-1} \vec{\rho}_{Y} & =\lambda_{S} \rho_{Y S}+\tilde{\rho}_{H Y}\left(\lambda_{H}-\rho_{H S} \lambda_{S}\right) .
\end{aligned}
$$


The Hamilton-Jacobi-Bellman (HJB) equation in this case can then be written as

$$
0=\mathcal{L}_{1} J+\mathcal{L}_{2} J+\mathcal{L}_{3} J+\mathcal{L}_{4} J
$$

where $\mathcal{L}_{1} J$ and $\mathcal{L}_{2} J$ are given by (37) and (38), and

$$
\begin{aligned}
& \mathcal{L}_{3} J=\frac{1}{2} \sigma_{H}^{2} h^{2} J_{h h}+\frac{1}{2} \sigma_{Y}^{2} y^{2} J_{y y}+\rho_{H Y} \sigma_{H} \sigma_{Y} h y J_{h y} \\
& \mathcal{L}_{4} J=\sup _{\Pi}\left\{J_{x} x \Pi^{\top} \mu+\frac{1}{2} J_{x x} x^{2} \Pi^{\top} \Sigma \Sigma^{\top} \Pi+J_{x h} x h \sigma_{H} \Pi^{\top} \Sigma \vec{\rho}_{H}+J_{x y} x y \sigma_{Y} \Pi^{\top} \Sigma \vec{\rho}_{Y}\right\}
\end{aligned}
$$

The first-order condition for $\Pi$ implies

$$
\Pi=-\frac{J_{x}}{x J_{x x}}\left(\Sigma \Sigma^{\top}\right)^{-1} \mu-\frac{y J_{x y}}{x J_{x x}} \sigma_{Y}\left(\Sigma^{\top}\right)^{-1} \vec{\rho}_{Y}-\frac{h J_{x h}}{x J_{x x}} \sigma_{H}\left(\Sigma^{\top}\right)^{-1} \vec{\rho}_{H} .
$$

Substituting this back into $\mathcal{L}_{4} J$, we find after long and tedious computations that

$$
\begin{aligned}
\mathcal{L}_{4} J= & -\frac{1}{2} \frac{J_{x}^{2}}{J_{x x}} \mu^{\top}\left(\Sigma \Sigma^{\top}\right)^{-1} \mu-\frac{1}{2} \frac{y^{2} J_{x y}^{2}}{J_{x x}} \sigma_{Y}^{2}-\frac{y J_{x} J_{x y}}{J_{x x}} \sigma_{Y} \mu^{\top}\left(\Sigma^{\top}\right)^{-1} \vec{\rho}_{Y} \\
& -\frac{1}{2} \frac{h^{2} J_{x h}^{2}}{J_{x x}} \sigma_{H}^{2}-\frac{h J_{x} J_{x h}}{J_{x x}} \sigma_{H} \mu^{\top}\left(\Sigma^{\top}\right)^{-1} \vec{\rho}_{H}-\frac{y h J_{x y} J_{x h}}{J_{x x}} \sigma_{Y} \sigma_{H} \rho_{Y H} .
\end{aligned}
$$

As in the deterministic model we conjecture that $J$ is of the form (5) for some functions $G, F, B$. The derivatives $J_{t}, J_{x}, J_{\bar{q}}, J_{y}$, and $J_{h}$ are as stated in Appendix B.1. In addition, we now need the following derivatives:

$$
\begin{aligned}
& J_{x x}=-\gamma(\chi h)^{k \gamma} G^{\gamma} \hat{x}^{-\gamma-1}, \\
& J_{y y}=-\gamma(\chi h)^{k \gamma} G^{\gamma} \hat{x}^{-\gamma-1} F^{2}, \\
& J_{h h}=k \gamma(\chi h)^{k \gamma} G^{\gamma} \hat{x}^{-\gamma} h^{-2}\left(\frac{k \gamma-1}{1-\gamma} \hat{x}-2 \bar{q} \chi h B+(\chi h \bar{q} B)^{2} k^{-1} \hat{x}^{-1}\right), \\
& J_{x y}=-\gamma(\chi h)^{k \gamma} G^{\gamma} \hat{x}^{-\gamma-1} F, \\
& J_{x h}=\gamma(\chi h)^{k \gamma} G^{\gamma} \hat{x}^{-\gamma-1} h^{-1}(k \hat{x}+\bar{q} \chi h B), \\
& J_{y h}=\gamma(\chi h)^{k \gamma} G^{\gamma} \hat{x}^{-\gamma-1} h^{-1} F(k \hat{x}+\bar{q} \chi h B) .
\end{aligned}
$$

Then

$$
\Pi=\frac{1}{\gamma} \frac{\hat{x}}{x}\left(\Sigma \Sigma^{\top}\right)^{-1} \mu-\frac{y F}{x} \sigma_{Y}\left(\Sigma^{\top}\right)^{-1} \vec{\rho}_{Y}+\left(k \frac{\hat{x}}{x}+\frac{\bar{q} \chi h B}{x}\right) \sigma_{H}\left(\Sigma^{\top}\right)^{-1} \vec{\rho}_{H},
$$


which leads to (17)-(18) by using (44)-(45). Furthermore,

$$
\begin{aligned}
& \mathcal{L}_{4} J=(\chi h)^{k \gamma} G^{\gamma} \hat{x}^{-\gamma-1}\left\{\hat{x}^{2}\left[\frac{1}{2 \gamma} \mu^{\top}\left(\Sigma \Sigma^{\top}\right)^{-1} \mu+\frac{k^{2} \gamma}{2} \sigma_{H}^{2}+k \sigma_{H} \mu^{\top}\left(\Sigma^{\top}\right)^{-1} \vec{\rho}_{H}\right]\right. \\
& +\hat{x}\left[\chi h \bar{q} B \sigma_{H}\left(k \gamma \sigma_{H}+\mu^{\top}\left(\Sigma^{\top}\right)^{-1} \vec{\rho}_{H}\right)\right. \\
& \left.-y F \sigma_{Y}\left(k \gamma \sigma_{H} \rho_{Y H}+\mu^{\top}\left(\Sigma^{\top}\right)^{-1} \vec{\rho}_{Y}\right)\right] \\
& \left.+\frac{\gamma}{2}(y F)^{2} \sigma_{Y}^{2}+\frac{\gamma}{2} \bar{q} \chi h B \sigma_{H}\left(\bar{q} \chi h B \sigma_{H}-2 y F \sigma_{Y} \rho_{Y H}\right)\right\}, \\
& \mathcal{L}_{3} J=(\chi h)^{k \gamma} G^{\gamma} \hat{x}^{-\gamma-1}\left\{\hat{x}^{2} \frac{k \gamma(k \gamma-1)}{2(1-\gamma)} \sigma_{H}^{2}+\hat{x} k \gamma \sigma_{H}\left[y F \rho_{H Y} \sigma_{Y}-\bar{q} \chi h B \sigma_{H}\right]\right. \\
& \left.-\frac{\gamma}{2} \sigma_{H}^{2} \chi^{2} h^{2} \bar{q}^{2} B^{2}-\frac{\gamma}{2} \sigma_{Y}^{2} y^{2} F^{2}+\gamma y F \chi h \bar{q} B \rho_{H Y} \sigma_{H} \sigma_{Y}\right\} .
\end{aligned}
$$

By adding up $\mathcal{L}_{3} J$ and $\mathcal{L}_{4} J$, various terms cancel, and we are left with

$$
\begin{aligned}
\mathcal{L}_{3} J+\mathcal{L}_{4} J=(\chi h)^{k \gamma} G^{\gamma} \hat{x}^{-\gamma}\{\hat{x} & {\left[\frac{1}{2 \gamma} \mu^{\top}\left(\Sigma \Sigma^{\top}\right)^{-1} \mu+\frac{k(k-1) \gamma}{2(1-\gamma)} \sigma_{H}^{2}+k \sigma_{H} \mu^{\top}\left(\Sigma^{\top}\right)^{-1} \vec{\rho}_{H}\right] } \\
& \left.+\chi h \bar{q} B \sigma_{H} \mu^{\top}\left(\Sigma^{\top}\right)^{-1} \vec{\rho}_{H}-y F \sigma_{Y} \mu^{\top}\left(\Sigma^{\top}\right)^{-1} \vec{\rho}_{Y}\right\} .
\end{aligned}
$$

If we substitute this as well as (40) and (41) into the HJB equation (48) and then divide by $(\chi h)^{k \gamma} G^{\gamma-1} \hat{x}^{-\gamma}$, each remaining term is either a multiple of $\hat{x}$ or does not involve $\hat{x}$ at all. Collecting the terms involving $\hat{x}$ we conclude that $G$ must satisfy

$$
0=G^{\prime}(t)-r_{G} G(t)+\hat{b}(1+\alpha B(t))^{k}
$$

where $r_{G}$ is now given by (14) if we use Eqs. (46)-(47). With $G(T)=0$ (due to no bequests), the solution is (7). The terms not involving $\hat{x}$ add up to zero if

$$
0=F^{\prime}(t)-r_{F}(t) F(t)+1, \quad 0=B^{\prime}(t)-r_{B} B(t)+1,
$$

where $r_{F}(t)$ and $r_{B}$ are given by (15) and (16). With $F(T)=B(T)=0$, the solutions are as stated in the theorem.

Before deriving the consumption dynamics, we consider the dynamics of wealth. By substitution of the optimal portfolio into the dynamics of tangible wealth, we find

$$
\begin{aligned}
d X_{t} & =\left\{X_{t}\left[r+\Pi_{t}^{\top} \mu\right]+Y_{t}-c_{t}-q_{t} \chi H_{t}\right\} d t+X_{t} \Pi_{t}^{\top} \Sigma d W_{t} \\
& =\left\{r X_{t}+\frac{1}{\gamma} \widehat{X}_{t} \Lambda^{2}-Y_{t} F(t) \sigma_{Y} \mu^{\top}\left(\Sigma^{\top}\right)^{-1} \vec{\rho}_{Y}\right.
\end{aligned}
$$




$$
\begin{aligned}
& \left.+\left(k+\bar{q}_{t} \chi H_{t} B(t)\right) \sigma_{H} \lambda_{H}+Y_{t}-c_{t}-q_{t} \chi H_{t}\right\} d t \\
+ & \left\{\frac{1}{\gamma} \widehat{X}_{t} \mu^{\top} \Sigma^{-1}-Y_{t} F(t) \sigma_{Y} \vec{\rho}_{Y}^{\top}+\left(k \widehat{X}_{t}+\bar{q}_{t} \chi H_{t} B(t)\right) \sigma_{H} \vec{\rho}_{H}\right\} d W_{t} .
\end{aligned}
$$

By applying (51) as well as the optimal consumption decisions (9), we find that the dynamics of disposable wealth are

$$
\begin{aligned}
d \widehat{X}_{t}= & d X_{t}+F(t) d Y_{t}+Y_{t} F^{\prime}(t) d t-\chi H_{t} B(t) d \bar{q}_{t}-\bar{q}_{t} \chi B(t) d H_{t}-\bar{q}_{t} \chi H_{t} B^{\prime}(t) d t \\
= & \widehat{X}_{t}\left(r+\frac{1}{\gamma} \Lambda^{2}+k \sigma_{H} \lambda_{H}\right) d t+\widehat{X}_{t}\left(\frac{1}{\gamma} \mu^{\top} \Sigma^{-1}+k \sigma_{H} \vec{\rho}_{H}^{\top}\right) d W_{t} \\
& -c_{t} d t-\left(q_{t}-\bar{q}_{t}\right) \chi H_{t}(1+\alpha B(t)) d t \\
= & \widehat{X}_{t}\left(r+\frac{1}{\gamma} \Lambda^{2}+k \sigma_{H} \lambda_{H}-\hat{b} \frac{(1+\alpha B(t))^{k}}{G(t)}\right) d t+\widehat{X}_{t}\left(\frac{1}{\gamma} \mu^{\top} \Sigma^{-1}+k \sigma_{H} \vec{\rho}_{H}^{\top}\right) d W_{t} .
\end{aligned}
$$

From (9) it follows that

$$
\begin{aligned}
d c_{t} & =c_{t} \frac{d \widehat{X}_{t}}{\widehat{X}_{t}}+c_{t}\left(\frac{k \alpha B^{\prime}(t)}{1+\alpha B(t)}-\frac{G^{\prime}(t)}{G(t)}\right) d t \\
& =c_{t} \frac{d \widehat{X}_{t}}{\widehat{X}_{t}}+c_{t}\left(\frac{k \alpha B^{\prime}(t)}{1+\alpha B(t)}-r_{G}+\hat{b} \frac{(1+\alpha B(t))^{k}}{G(t)}\right) d t \\
& =c_{t}\left(K_{c}+\frac{k \alpha B^{\prime}(t)}{1+\alpha B(t)}\right) d t+\left(\frac{1}{\gamma} \mu^{\top} \Sigma^{-1}+k \sigma_{H} \vec{\rho}_{H}^{\top}\right) d W_{t}
\end{aligned}
$$

where we have used (42) and $K_{c}$ is defined by (20). The expected consumption is

$$
\mathrm{E}\left[c_{t}\right]=c_{0} e^{\int_{0}^{t} \mu_{c}(s) d s}, \quad \mu_{c}(t)=K_{c}+\frac{k \alpha B^{\prime}(t)}{1+\alpha B(t)}=K_{c}-\frac{k \alpha r_{B}}{\left(r_{B}+\alpha\right) e^{r_{B}(T-t)}-\alpha} .
$$

Note that

$$
\frac{d \mathrm{E}\left[c_{t}\right]}{d t}=\mu_{c}(t) \mathrm{E}\left[c_{t}\right] d t
$$

with the sign determined exclusively by $\mu_{c}(t)$. Since $B^{\prime}(t)=-e^{-r_{B}(T-t)}<0$ and $B^{\prime \prime}(t)=$ $r_{B} B^{\prime}(t)=-r_{B} e^{-r_{B}(T-t)}$, we get

$$
\mu_{c}^{\prime}(t)=k \alpha \frac{B^{\prime \prime}(t)[1+\alpha B(t)]-\alpha\left[B^{\prime}(t)\right]^{2}}{(1+\alpha B(t))^{2}}=k \alpha \frac{\left(r_{B}+\alpha\right) B^{\prime}(t)}{(1+\alpha B(t))^{2}}=k \alpha \frac{(\varepsilon+\chi-m) B^{\prime}(t)}{(1+\alpha B(t))^{2}},
$$

which is negative if $\varepsilon+\chi-m>0$. Then we have a hump in expected consumption provided $\mu_{c}(0)>0$ and $\mu_{c}(T)<0$, which is the case exactly when (19) holds, and the hump occurs when $\mu_{c}(t)=0$ which implies (21). 


\section{A.3 Proof of Lemma 1}

Let $\tilde{X}_{t}$ denote the tangible wealth in the artificial market. In analogy with (13), the wealth dynamics generated by a strategy $\left(c, q, \Pi_{S}, \Pi_{H}, \Pi_{I}\right)$ are

$$
\begin{aligned}
& d \tilde{X}_{t}=\left\{\tilde{X}_{t}\left[\tilde{r}(t)+\Pi_{S t} \tilde{\mu}_{S}(t)+\Pi_{H t}\left(\tilde{\mu}_{H}(t)+\tilde{\chi}(t)-\tilde{m}(t)\right)+\Pi_{I t} \nu_{I}(t)\right]\right. \\
&\left.+Y_{t}-c_{t}-q_{t} \tilde{\chi}(t) \tilde{H}_{t}\right\} d t+\tilde{X}_{t}\left(\Pi_{S t} \sigma_{S}+\Pi_{H t} \sigma_{H} \rho_{H S}+\Pi_{I t} \rho_{I S}\right) d W_{S t} \\
&+ \tilde{X}_{t}\left(\Pi_{H t} \sigma_{H} \sqrt{1-\rho_{H S}^{2}}+\Pi_{I t} \hat{\rho}_{I H}\right) d W_{H t}+\tilde{X}_{t} \Pi_{I t} \hat{\rho}_{I} d W_{Y t} \\
&=\left\{\tilde{X}_{t}\left[\tilde{r}(t)+\Pi_{S t} \tilde{\mu}_{S}(t)+\Pi_{H t}\left(\tilde{\mu}_{H}(t)+\chi-m\right)+\Pi_{I t} \nu_{I}(t)\right]\right. \\
&\left.+Y_{t}-c_{t}-q_{t} \chi H_{t}\right\} d t+\tilde{X}_{t}\left(\Pi_{S t} \sigma_{S}+\Pi_{H t} \sigma_{H} \rho_{H S}+\Pi_{I t} \rho_{I S}\right) d W_{S t} \\
&+ \tilde{X}_{t}\left(\Pi_{H t} \sigma_{H} \sqrt{1-\rho_{H S}^{2}}+\Pi_{I t} \hat{\rho}_{I H}\right) d W_{H t}+\tilde{X}_{t} \Pi_{I t} \hat{\rho}_{I} d W_{Y t},
\end{aligned}
$$

where the equality follows from $\tilde{\chi}(t) \tilde{H}_{t}=\chi H_{t}$ and $\tilde{\chi}(t)-\tilde{m}(t)=\chi-m$, which are true by construction. In particular, if we take a strategy $\left(c, q, \Pi_{S}, \Pi_{H}\right)$ which is feasible in the true market and let $\Pi_{I t} \equiv 0$, we have

$$
\begin{aligned}
d \tilde{X}_{t}= & \left\{\tilde{X}_{t}\left[\tilde{r}(t)+\Pi_{S t} \tilde{\mu}_{S}(t)+\Pi_{H t}\left(\tilde{\mu}_{H}(t)+\chi-m\right)\right]+Y_{t}-c_{t}-q_{t} \chi H_{t}\right\} d t \\
& +\tilde{X}_{t}\left(\Pi_{S t} \sigma_{S}+\Pi_{H t} \sigma_{H} \rho_{H S}\right) d W_{S t}+\tilde{X}_{t} \Pi_{H t} \sigma_{H} \sqrt{1-\rho_{H S}^{2}} d W_{H t}
\end{aligned}
$$

A comparison with (13) reveals that the strategy leads to at least the same wealth in the artificial market as in the true market provided that

$$
\tilde{r}(t)+\Pi_{S t} \tilde{\mu}_{S}(t)+\Pi_{H t} \tilde{\mu}_{H}(t) \geq r+\Pi_{S t} \mu_{S}+\Pi_{H t} \mu_{H}
$$

or, equivalently,

$$
\Pi_{S t} \nu_{S}(t)+\Pi_{H t} \nu_{H}(t)+\max \left(\nu_{S}(t)^{-}, \frac{1}{\kappa} \nu_{H}(t)^{-}\right) \geq 0
$$

for any $\left(\nu_{S}(t), \nu_{H}(t)\right)$. We verify $(52)$ in the following cases that cover all situations:

(i) $\nu_{S}(t), \nu_{H}(t) \geq 0$ : obvious as all terms on left-hand side are non-negative

(ii) $\nu_{H}(t)<0 \leq \nu_{S}(t)$ : the left-hand side is then

$$
\Pi_{S t} \nu_{S}(t)+\Pi_{H t} \nu_{H}(t)-\frac{1}{\kappa} \nu_{H}(t)=\Pi_{S t} \nu_{S}(t)-\frac{1}{\kappa} \nu_{H}(t)\left(1-\kappa \Pi_{H t}\right) \geq 0
$$


(iii) $\nu_{S}(t)<0 \leq \nu_{H}(t)$ : the left-hand side is then

$$
\Pi_{S t} \nu_{S}(t)+\Pi_{H t} \nu_{H}(t)-\nu_{S}(t)=-\nu_{S}(t)\left(1-\Pi_{S t}\right)+\Pi_{H t} \nu_{H}(t) \geq 0
$$

(iv) $\nu_{S}(t) \leq \frac{1}{\kappa} \nu_{H}(t) \leq 0$ : the left-hand side is then

$$
\begin{aligned}
\Pi_{S t} \nu_{S}(t)+\Pi_{H t} \nu_{H}(t) & -\nu_{S}(t) \\
& =-\nu_{S}(t)\left(1-\Pi_{S t}-\kappa \Pi_{H t}\right)+\kappa \Pi_{H t}\left(\frac{1}{\kappa} \nu_{H}(t)-\nu_{S}(t)\right) \geq 0
\end{aligned}
$$

(v) $\frac{1}{\kappa} \nu_{H}(t) \leq \nu_{S}(t) \leq 0$ : the left-hand side is then

$$
\begin{aligned}
\Pi_{S t} \nu_{S}(t)+\Pi_{H t} \nu_{H}(t)- & \frac{1}{\kappa} \nu_{H}(t) \\
& =\Pi_{S t}\left(\nu_{S}(t)-\frac{1}{\kappa} \nu_{H}(t)\right)-\frac{1}{\kappa} \nu_{H}(t)\left(1-\Pi_{S t}-\kappa \Pi_{H t}\right) \geq 0 .
\end{aligned}
$$

\section{A.4 Proof of Theorem 3}

In this appendix we consider a given artificial market as represented by a fixed $\theta$, cf. (28). For simplicity we notationally suppress $\theta$. The proof is a relatively straightforward extension of the proof of Theorem 2 presented in Appendix A.2. Compared to that case, we now have an additional risky asset, namely the income derivative.

\section{A.4.1 Setting and notation}

The investor has access to a risk-free asset with a instantaneous rate of return $\tilde{r}(t)$ and three risky assets with price dynamics

$$
d P_{t}=\operatorname{diag}\left(P_{t}\right)\left[(\tilde{r}(t) \mathbf{1}+\tilde{\mu}(t))+\Sigma d W_{t}\right],
$$

where $W=\left(W_{S}, W_{H}, W_{Y}\right)^{\top}$ is a three-dimensional standard Brownian motion (with independent components), and

$$
\tilde{\mu}(t)=\left(\begin{array}{c}
\tilde{\mu}_{S}(t) \\
\tilde{\mu}_{H}(t)+\chi-m \\
\nu_{I}(t)
\end{array}\right), \quad \Sigma=\left(\begin{array}{ccc}
\sigma_{S} & 0 & 0 \\
\sigma_{H} \rho_{H S} & \sigma_{H} \sqrt{1-\rho_{H S}^{2}} & 0 \\
\rho_{I S} & \hat{\rho}_{I H} & \hat{\rho}_{I}
\end{array}\right) .
$$

The net rental rate $\tilde{\chi}(t)-\tilde{m}(t)=\chi-m$ is compounded into the house price dynamics to reflect the total return on a housing investment. We introduce the correlation vectors

$$
\vec{\rho}_{Y}=\left(\begin{array}{lll}
\rho_{Y S} & \hat{\rho}_{Y H} & \hat{\rho}_{Y}
\end{array}\right)^{\top}, \quad \vec{\rho}_{H}=\left(\begin{array}{lll}
\rho_{H S} & \sqrt{1-\rho_{H S}^{2}} & 0
\end{array}\right)^{\top} .
$$


For future use, we note that

$$
\begin{aligned}
& \Sigma^{-1}=\left(\begin{array}{ccc}
\frac{1}{\sigma_{S}} & 0 & 0 \\
-\frac{\rho_{H S}}{\sigma_{S} \sqrt{1-\rho_{H S}^{2}}} & \frac{1}{\sigma_{H} \sqrt{1-\rho_{H S}^{2}}} & 0 \\
\frac{\rho_{H S} \hat{\rho}_{H I}-\rho_{I S} \sqrt{1-\rho_{H S}^{2}}}{\sigma_{S} \sqrt{1-\rho_{H S}^{2}} \hat{\rho}_{I}} & -\frac{\hat{\rho}_{I H}}{\sigma_{H} \sqrt{1-\rho_{H S}^{2}} \hat{\rho}_{I}} & \frac{1}{\hat{\rho}_{I}}
\end{array}\right), \\
& \tilde{\mu}(t)^{\top}\left(\Sigma \Sigma^{\top}\right)^{-1}=\left(\begin{array}{lll}
\frac{\xi_{S}(t)}{\sigma_{S}} & \frac{\xi_{H}(t)}{\sigma_{H}} & \xi_{I}(t)
\end{array}\right), \\
& \tilde{\Lambda}(t)^{2} \equiv \tilde{\mu}(t)^{\top}\left(\Sigma \Sigma^{\top}\right)^{-1} \tilde{\mu}(t)=\xi_{S}(t) \tilde{\lambda}_{S}(t)+\xi_{H}(t) \tilde{\lambda}_{H}(t)+\xi_{I}(t) \nu_{I}(t), \\
& \vec{\rho}_{Y}^{\top} \Sigma^{-1}=\left(\begin{array}{lll}
\frac{\psi_{S}}{\sigma_{S}} & \frac{\psi_{H}}{\sigma_{H}} & \psi_{Y}
\end{array}\right), \\
& \vec{\rho}_{H}^{\top} \Sigma^{-1}=\left(\begin{array}{lll}
0 & \frac{1}{\sigma_{H}} & 0
\end{array}\right),
\end{aligned}
$$

where

$$
\begin{aligned}
\xi_{S}(t) & =\tilde{\lambda}_{S}(t) \frac{1-\rho_{I H}^{2}}{\left(1-\rho_{H S}^{2}\right) \hat{\rho}_{I}^{2}}-\tilde{\lambda}_{H}(t) \frac{\rho_{H S}-\rho_{I S} \rho_{I H}}{\left(1-\rho_{H S}^{2}\right) \hat{\rho}_{I}^{2}}-\nu_{I}(t) \frac{\rho_{I S}-\rho_{H S} \rho_{I H}}{\left(1-\rho_{H S}^{2}\right) \hat{\rho}_{I}^{2}}, \\
\xi_{H}(t) & =-\tilde{\lambda}_{S}(t) \frac{\rho_{H S}-\rho_{I S} \rho_{I H}}{\left(1-\rho_{H S}^{2}\right) \hat{\rho}_{I}^{2}}+\tilde{\lambda}_{H}(t) \frac{1-\rho_{I S}^{2}}{\left(1-\rho_{H S}^{2}\right) \hat{\rho}_{I}^{2}}-\nu_{I}(t) \frac{\rho_{I H}-\rho_{H S} \rho_{I S}}{\left(1-\rho_{H S}^{2}\right) \hat{\rho}_{I}^{2}}, \\
\xi_{I}(t) & =-\tilde{\lambda}_{S}(t) \frac{\rho_{I S}-\rho_{H S} \rho_{I H}}{\left(1-\rho_{H S}^{2}\right) \hat{\rho}_{I}^{2}}-\tilde{\lambda}_{H}(t) \frac{\rho_{I H}-\rho_{H S} \rho_{I S}}{\left(1-\rho_{H S}^{2}\right) \hat{\rho}_{I}^{2}}+\nu_{I}(t) \frac{1}{\hat{\rho}_{I}^{2}}, \\
\psi_{Y} & =\frac{\hat{\rho}_{Y}}{\hat{\rho}_{I}}, \quad \psi_{H}=\frac{\hat{\rho}_{Y H}-\hat{\rho}_{I H} \psi_{Y}}{\sqrt{1-\rho_{H S}^{2}}}, \quad \psi_{S}=\rho_{Y S}-\rho_{H S} \psi_{H}-\rho_{I S} \psi_{Y} .
\end{aligned}
$$

\section{A.4.2 Human capital}

The artificial market is complete so a unique risk-neutral measure $\mathbb{Q}$ exists, and the human capital can be valued as if the income was a dividend stream:

$$
\mathcal{H}(t, y)=\mathrm{E}^{\mathbb{Q}}\left[\int_{t}^{T} Y_{s} e^{-\int_{t}^{s} \tilde{r}(u) d u} d s \mid Y_{t}=y\right] .
$$

We need the risk-neutral dynamics of $Y_{t}$. The market price of risk is $\Sigma^{-1} \tilde{\mu}(t)$ so

$$
W_{t}^{\mathbb{Q}}=W_{t}+\int_{0}^{t} \Sigma^{-1} \tilde{\mu}(s) d s
$$

defines a standard Brownian motion under $\mathbb{Q}$. Therefore

$$
d Y_{t}=Y_{t}\left[\left(\mu_{Y}(t)-\sigma_{Y}(t) \vec{\rho}^{\top} \Sigma^{-1} \tilde{\mu}(t)\right) d t+\sigma_{Y}(t) \vec{\rho}^{\top} d W_{t}^{\mathbb{Q}}\right] .
$$


It follows from standard asset pricing theory that $\mathcal{H}(t, y)$ satisfies the PDE

$$
\tilde{r}(t) \mathcal{H}=\mathcal{H}_{t}+\mathcal{H}_{y} y\left(\mu_{Y}(t)-\sigma_{Y}(t) \vec{\rho}^{\top} \Sigma^{-1} \tilde{\mu}(t)\right)+\frac{1}{2} \mathcal{H}_{y y} y^{2} \sigma_{Y}(t)^{2}+y .
$$

First consider the case after retirement, $t>\tilde{T}$, where the terminal value is $\mathcal{H}(T, y)=0$. The solution is of the form $\mathcal{H}(t, y)=y F(t)$ if $F$ satisfies the ODE

$$
F^{\prime}(t)-r_{F}(t) F(t)+1=0
$$

and $F(T)=0$, where

$$
\begin{aligned}
r_{F}(t) & =\tilde{r}(t)-\mu_{Y}(t)+\sigma_{Y}(t) \vec{\rho}^{\top} \Sigma^{-1} \tilde{\mu}(t) \\
& =\tilde{r}(t)-\mu_{Y}(t)+\sigma_{Y}(t)\left(\psi_{S} \tilde{\lambda}_{S}(t)+\psi_{H} \tilde{\lambda}_{H}(t)+\psi_{Y} \nu_{I}(t)\right),
\end{aligned}
$$

using (55). The solution is $F(t)=\int_{t}^{T} \exp \left\{-\int_{t}^{u} r_{F}(s) d s\right\} d u$.

Before retirement, $t<\tilde{T}$, we need to take the income drop at retirement into account. The human capital still solves the above PDE but now with terminal condition $\mathcal{H}(\tilde{T}, y)=$ $\Upsilon Y_{\tilde{T}-} F(\tilde{T})$. The solution is then $\mathcal{H}(t, y)=y F(t)$ with

$$
F(t)=\int_{t}^{\tilde{T}} \exp \left\{-\int_{t}^{u} r_{F}(s) d s\right\} d u+\Upsilon \int_{\tilde{T}}^{T} \exp \left\{-\int_{t}^{u} r_{F}(s) d s\right\} d u .
$$

\section{A.4.3 The HJB equation and its solution}

We write the portfolio strategy in the artificial market compactly as $\Pi=\left(\Pi_{S}, \Pi_{H}, \Pi_{I}\right)^{\top}$. The wealth dynamics are generated by a strategy $\left(c, q, \Pi_{S}, \Pi_{H}, \Pi_{I}\right)$ are

$$
\begin{aligned}
d X_{t} & =\left\{X_{t}\left[\tilde{r}(t)+\Pi_{t}^{\top} \tilde{\mu}(t)\right]+Y_{t}-c_{t}-q_{t} \tilde{\chi}(t) \tilde{H}_{t}\right\} d t+X_{t} \Pi_{t}^{\top} \Sigma d W_{t} \\
& =\left\{X_{t}\left[\tilde{r}(t)+\Pi_{t}^{\top} \tilde{\mu}(t)\right]+Y_{t}-c_{t}-q_{t} \chi H_{t}\right\} d t+X_{t} \Pi_{t}^{\top} \Sigma d W_{t},
\end{aligned}
$$

where the equality is due to $\tilde{\chi}(t) \tilde{H}_{t}=\chi H_{t}$ by construction of $\tilde{\chi}(t)$. We see that $\left(t, X_{t}, Y_{t}, H_{t}\right)$ constitutes a controlled Markov diffusion so that these quantities are the relevant state variables for the agent with the addition of the housing habit $\bar{q}_{t}$ that directly affects the agent's utility. The dynamics of the state variables $Y_{t}, H_{t}$, and $\bar{q}_{t}$ are given by (22), (11), and (1), respectively.

The HJB equation for the value function $J(t, x, y, h, \bar{q})$ in the artificial market can be written as

$$
0=\mathcal{L}_{1} J+\mathcal{L}_{2} J+\mathcal{L}_{3} J+\mathcal{L}_{4} J
$$


where

$$
\begin{aligned}
& \mathcal{L}_{1} J=\sup _{c, q}\left\{-c J_{x}-(q-\bar{q}) \chi h J_{x}+\frac{1}{1-\gamma} c^{b(1-\gamma)}(q-\bar{q})^{(1-b)(1-\gamma)}+\alpha(q-\bar{q}) J_{\bar{q}}\right\}, \\
& \mathcal{L}_{2} J=J_{t}+\tilde{r} x J_{x}+y J_{x}+\left(r+\mu_{H}\right) h J_{h}+\mu_{Y} y J_{y}-\bar{q} \chi h J_{x}+(\alpha-\varepsilon) \bar{q} J_{\bar{q}}-\delta J, \\
& \mathcal{L}_{3} J=\frac{1}{2} \sigma_{H}^{2} h^{2} J_{h h}+\frac{1}{2} \sigma_{Y}^{2} y^{2} J_{y y}+\rho_{H Y} \sigma_{H} \sigma_{Y} h y J_{h y}, \\
& \mathcal{L}_{4} J=\sup _{\Pi}\left\{J_{x} x \Pi^{\top} \tilde{\mu}+\frac{1}{2} J_{x x} x^{2} \Pi^{\top} \Sigma \Sigma^{\top} \Pi+J_{x h} x h \sigma_{H} \Pi^{\top} \Sigma \vec{\rho}_{H}+J_{x y} x y \sigma_{Y} \Pi^{\top} \Sigma \vec{\rho}_{Y}\right\} .
\end{aligned}
$$

Note that $\mathcal{L}_{1} J$ and $\mathcal{L}_{2} J$ are exactly as in the simpler models, cf. (37)-(38), except that $\tilde{r}$ replaces $r$ in front of $x J_{x}$. Furthermore, $\mathcal{L}_{3} J$ is exactly as in the spanned risk model of Section 4.2 , cf. (49). In addition, $\mathcal{L}_{4} J$ has the same structure as (50) in the spanned risk model, but $\mu$ is replaced by $\tilde{\mu}, \Pi$ is now three-dimensional because of the additional risky asset, and $\vec{\rho}_{H}$ and $\vec{\rho}_{Y}$ are also three-dimensional.

Just as in the simpler models we conjecture a solution of the form

$$
J(t, x, y, h, \bar{q})=\frac{1}{1-\gamma}(\chi h)^{k \gamma} G(t)^{\gamma} \hat{x}^{1-\gamma}, \quad \hat{x}=x+y F(t)-\bar{q} \chi h B(t) .
$$

As in the proof of Theorem 1 (see Appendix B.1), this implies the optimal consumption policy (31)-(32) and, suppressing $t$-dependence for clarity,

$$
\begin{aligned}
& \mathcal{L}_{1} J=\hat{x}^{1-\gamma}(\chi h)^{\gamma k} G^{\gamma-1} \frac{\gamma}{1-\gamma} \hat{b}(1+\alpha B)^{k} \\
& \mathcal{L}_{2} J=\hat{x}^{-\gamma}(\chi h)^{\gamma k} G^{\gamma-1}\left\{\frac{\gamma}{1-\gamma} \hat{x}\left[G^{\prime}-\left(\frac{\delta}{\gamma}+\frac{\gamma-1}{\gamma} \tilde{r}-\left(r+\mu_{H}\right) k\right) G\right]\right. \\
&\left.+G y\left[F^{\prime}+\left(\mu_{Y}-\tilde{r}\right) F+1\right]-\bar{q} \chi h G\left[B^{\prime}-\left(\tilde{r}-r-\mu_{H}+\varepsilon-\alpha\right) B+1\right]\right\} .
\end{aligned}
$$

As in the proof of Theorem 2 (see Appendix A.2), we find

$$
\begin{aligned}
\mathcal{L}_{3} J=\hat{x}^{-\gamma-1}(\chi h)^{k \gamma} G^{\gamma}\{ & \hat{x}^{2} \frac{k \gamma(k \gamma-1)}{2(1-\gamma)} \sigma_{H}^{2}+\hat{x} k \gamma \sigma_{H}\left[y F \rho_{H Y} \sigma_{Y}-\bar{q} \chi h B \sigma_{H}\right] \\
& \left.-\frac{\gamma}{2} \sigma_{H}^{2} h^{2} \chi^{2} \bar{q}^{2} B^{2}-\frac{\gamma}{2} \sigma_{Y}^{2} y^{2} F^{2}+\gamma y F h \chi \bar{q} B \rho_{H Y} \sigma_{H} \sigma_{Y}\right\} .
\end{aligned}
$$

Regarding $\mathcal{L}_{4} J$, the first-order condition implies for $\Pi$ implies

$$
\Pi=-\frac{J_{x}}{x J_{x x}}\left(\Sigma \Sigma^{\top}\right)^{-1} \tilde{\mu}-\frac{y J_{x y}}{x J_{x x}} \sigma_{Y}\left(\Sigma^{\top}\right)^{-1} \vec{\rho}_{Y}-\frac{\omega h J_{x h}}{x J_{x x}} \sigma_{H}\left(\Sigma^{\top}\right)^{-1} \vec{\rho}_{H} .
$$


By our conjecture for $J$, we get

$$
-\frac{J_{x}}{x J_{x x}}=\frac{\hat{x}}{\gamma x}, \quad-\frac{y J_{x y}}{x J_{x x}}=-\frac{y F}{x}, \quad-\frac{h J_{x h}}{x J_{x x}}=\frac{k \hat{x}+\bar{q} \chi h B}{x},
$$

which, together with (53)-(56), implies that the optimal investment strategy can be written as (33)-(35). Substituting the above expression for $\Pi$ back into $\mathcal{L}_{4} J$, we obtain

$$
\begin{aligned}
\mathcal{L}_{4} J= & -\frac{1}{2} \frac{J_{x}^{2}}{J_{x x}} \tilde{\mu}(t)^{\top}\left(\Sigma \Sigma^{\top}\right)^{-1} \tilde{\mu}-\frac{1}{2} \frac{y^{2} J_{x y}^{2}}{J_{x x}} \sigma_{Y}^{2}-\frac{y J_{x} J_{x y}}{J_{x x}} \sigma_{Y} \tilde{\mu}^{\top}\left(\Sigma^{\top}\right)^{-1} \vec{\rho}_{Y} \\
& -\frac{1}{2} \frac{h^{2} J_{x h}^{2}}{J_{x x}} \sigma_{H}^{2}-\frac{h J_{x} J_{x h}}{J_{x x}} \sigma_{H} \tilde{\mu}^{\top}\left(\Sigma^{\top}\right)^{-1} \vec{\rho}_{H}-\frac{y h J_{x y} J_{x h}}{J_{x x}} \sigma_{Y} \sigma_{H} \rho_{Y H},
\end{aligned}
$$

and by substitution of our conjecture for $J$ this leads to

$$
\begin{aligned}
& \mathcal{L}_{4} J=(\chi h)^{k \gamma} G^{\gamma} \hat{x}^{-\gamma-1}\left\{\hat{x}^{2}\left[\frac{1}{2 \gamma} \tilde{\mu}^{\top}\left(\Sigma \Sigma^{\top}\right)^{-1} \tilde{\mu}+\frac{k^{2} \gamma}{2} \sigma_{H}^{2}+k \sigma_{H} \tilde{\mu}^{\top}\left(\Sigma^{\top}\right)^{-1} \vec{\rho}_{H}\right]\right. \\
& +\hat{x}\left[\bar{q} \chi h B \sigma_{H}\left(k \gamma \sigma_{H}+\tilde{\mu}^{\top}\left(\Sigma^{\top}\right)^{-1} \vec{\rho}_{H}\right)\right. \\
& \left.-y F \sigma_{Y}\left(k \gamma \sigma_{H} \rho_{Y H}+\tilde{\mu}^{\top}\left(\Sigma^{\top}\right)^{-1} \vec{\rho}_{Y}\right)\right] \\
& \left.+\frac{\gamma}{2}(y F)^{2} \sigma_{Y}^{2}+\frac{\gamma}{2} \bar{q} \chi h B \sigma_{H}\left(\bar{q} \chi h B \sigma_{H}-2 y F \sigma_{Y} \rho_{Y H}\right)\right\} \text {. }
\end{aligned}
$$

By adding up $\mathcal{L}_{3} J$ and $\mathcal{L}_{4} J$, various terms cancel, and we are left with

$$
\begin{aligned}
\mathcal{L}_{3} J+\mathcal{L}_{4} J=(\chi h)^{k \gamma} G^{\gamma} \hat{x}^{-\gamma}\{\hat{x} & {\left[\frac{1}{2 \gamma} \tilde{\mu}^{\top}\left(\Sigma \Sigma^{\top}\right)^{-1} \tilde{\mu}+\frac{k(k-1) \gamma}{2(1-\gamma)} \sigma_{H}^{2}+k \sigma_{H} \tilde{\mu}^{\top}\left(\Sigma^{\top}\right)^{-1} \vec{\rho}_{H}\right] } \\
& \left.+\bar{q} \chi h B \sigma_{H} \tilde{\mu}^{\top}\left(\Sigma^{\top}\right)^{-1} \vec{\rho}_{H}-y F \sigma_{Y} \tilde{\mu}^{\top}\left(\Sigma^{\top}\right)^{-1} \vec{\rho}_{Y}\right\} .
\end{aligned}
$$

By adding $\mathcal{L}_{1} J$ and $\mathcal{L}_{2} J$ to the above and equating the sum to zero, we have the full HJB equation. After division by $(\chi h)^{k \gamma} G^{\gamma} \hat{x}^{-\gamma}$, each remaining term is either a multiple of $\hat{x}$ or does not involve $\hat{x}$ at all. Collecting the terms with $\hat{x}$, we see that $G$ must satisfy

$$
0=G^{\prime}(t)-r_{G}(t) G(t)+\hat{b}(1+\alpha B(t))^{k}
$$

where

$$
\begin{aligned}
r_{G}(t) & =\frac{\delta}{\gamma}+\frac{\gamma-1}{\gamma} \tilde{r}(t)+\frac{\gamma-1}{2 \gamma^{2}} \tilde{\Lambda}(t)^{2}-k\left[r+\mu_{H}+\frac{k-1}{2} \sigma_{H}^{2}-\frac{\gamma-1}{\gamma} \sigma_{H} \tilde{\mu}^{\top}\left(\Sigma^{\top}\right)^{-1} \vec{\rho}_{H}\right] \\
& =\frac{\delta}{\gamma}+\frac{\gamma-1}{\gamma} \tilde{r}(t)+\frac{\gamma-1}{2 \gamma^{2}} \tilde{\Lambda}(t)^{2}
\end{aligned}
$$




$$
\begin{aligned}
& -k\left[\tilde{r}(t)-r_{\omega}(t)+\nu_{H}+\mu_{H}+\frac{k-1}{2} \sigma_{H}^{2}-\frac{\gamma-1}{\gamma}\left(\tilde{\mu}_{H}(t)+\chi-m\right)\right] \\
=\frac{\delta}{\gamma}+\frac{\gamma-1}{\gamma} \tilde{r}(t)+\frac{\gamma-1}{2 \gamma^{2}} \tilde{\Lambda}(t)^{2} & \\
& -k\left[\tilde{r}(t)-r_{\omega}(t)+\frac{\tilde{\mu}_{H}(t)}{\gamma}-\frac{\gamma-1}{\gamma}(\chi-m)+\frac{k-1}{2} \sigma_{H}^{2}\right] .
\end{aligned}
$$

The terms not involving $\hat{x}$ add up to zero provided

$$
0=F^{\prime}(t)-r_{F}(t) F(t)+1, \quad 0=B^{\prime}(t)-r_{B}(t) B(t)+1
$$

where

$$
\begin{aligned}
r_{F}(t) & =\tilde{r}(t)-\mu_{Y}(t)+\sigma_{Y}(t) \tilde{\mu}(t)^{\top}\left(\Sigma^{\top}\right)^{-1} \vec{\rho}_{Y} \\
& =\tilde{r}(t)-\mu_{Y}(t)+\sigma_{Y}(t)\left(\psi_{S} \tilde{\lambda}_{S}(t)+\psi_{H} \tilde{\lambda}_{H}(t)+\psi_{Y} \nu_{I}(t)\right)
\end{aligned}
$$

as in Appendix A.4.2, and

$$
\begin{aligned}
r_{B}(t) & =\tilde{r}(t)-r-\mu_{H}+\varepsilon-\alpha+\sigma_{H} \tilde{\mu}(t)^{\top}\left(\Sigma^{\top}\right)^{-1} \vec{\rho}_{H} \\
& =r_{\omega}(t)-\nu_{H}(t)-\mu_{H}+\varepsilon-\alpha+\tilde{\mu}_{H}(t)+\chi-m \\
& =r_{\omega}(t)+\varepsilon-\alpha+\chi-m .
\end{aligned}
$$

Note that the ODE for $F$ is satisfied according to our computation of the human capital, cf. Eq. (61). With the relevant boundary conditions $G(T)=B(T)=0$, the solutions to the ODEs for $G$ and $B$ are as stated in the theorem.

\section{B Extensions to bequest and mortality risk}

Let $\zeta(t)$ denote the mortality intensity. We assume the agent can invest in an insurance contract that pays the agent a continuous stream at the rate $\Gamma \zeta(t) N$ as long as the agent survives but, in return, when the agent dies the company receives an amount of $N$ out or the wealth of the agent. Here $N$ is a choice variable of the agent, whereas $\Gamma$ is an exogenously given constant with $\Gamma=1$ corresponding to a fairly priced contract.

In all our cases this adds two terms to the HJB equation:

(i) The term $\Gamma \zeta(t) N J_{x}$ due to the insurance flow payment $\Gamma \zeta(t) N$.

(ii) The term $\zeta(t)\left(w^{\gamma} \frac{1}{1-\gamma}(x-N)^{1-\gamma}-J\right)$ reflecting the jump in utility upon death multiplied by the death intensity.

Here $J$ and $x$ refer to values immediately before time $t$. The agent cannot live longer than $T$. The optimal insurance goes to zero as time approaches $T$. 


\section{B.1 The model of Section 4.1}

For the simple model of Section 4.1, the preferences are extended to include a power utility of terminal tangible wealth, representing a bequest:

$$
J(t, x, h, y, \bar{q})=\sup _{c, q, N} \mathrm{E}_{t}\left[\int_{t}^{\tau} e^{-\delta(s-t)} U\left(c_{s}, q_{s}, \bar{q}_{s}\right) d s+w^{\gamma} e^{-\delta(\tau-t)} \frac{1}{1-\gamma}\left(X_{\tau-}-N\right)^{1-\gamma}\right],
$$

where the expectation is introduced because of the mortality risk and $\tau \leq T$ denotes the random terminal date. The terminal condition at $T$ is $J(T, x, h, y, \bar{q})=w^{\gamma} \frac{1}{1-\gamma} x^{1-\gamma}$.

Theorem 4 The value function is

$$
J(t, x, h, y, \bar{q})=\frac{1}{1-\gamma}\left(w A(t)+(\chi h)^{k} G(t)\right)^{\gamma}(x+y F(t)-\bar{q} h \chi B(t))^{1-\gamma},
$$

where

$$
\begin{gathered}
A(t)=e^{-\int_{t}^{T} r_{A}(s) d s}+\int_{t}^{T} e^{-\int_{t}^{u} r_{A}(s) d s} \zeta(u) d u, \\
B(t)=\int_{t}^{T} e^{-\int_{t}^{s} r_{B}(u) d u} d s, \\
G(t)=\hat{b} \int_{t}^{T} e^{-\int_{t}^{s} r_{G}(u) d u}(1+\alpha B(s))^{k} d s, \\
F(t)= \begin{cases}\int_{t}^{T} e^{-\int_{t}^{u} r_{F}(s) d s} d u, & t \in[\tilde{T}, T], \\
\int_{t}^{\tilde{T}} e^{-\int_{t}^{u} r_{F}(s) d s} d u+\Upsilon \int_{\tilde{T}}^{T} e^{-\int_{t}^{u} r_{F}(s) d s} d u, & t<\tilde{T}, \\
r_{A}(t)=\frac{\delta+\zeta(t)}{\gamma}+\frac{\gamma-1}{\gamma}(r+\Gamma \zeta(t)), & r_{G}(t)=r_{A}(t)-\left(r+\mu_{H}\right) k, \\
r_{B}(t)=\Gamma \zeta(t)+\varepsilon-\alpha-\mu_{H}, & r_{F}(t)=r+\Gamma \zeta(t)-\mu_{Y}(t) .\end{cases}
\end{gathered}
$$

The optimal perishable and housing consumption rates are

$$
c_{t}=b \hat{b} \frac{\left(\chi H_{t}\right)^{k}(1+\alpha B(t))^{k}}{w A(t)+\left(\chi H_{t}\right)^{k} G(t)} \widehat{X}_{t}, \quad q_{t}=\bar{q}_{t}+(1-b) \hat{b} \frac{\left(\chi H_{t}\right)^{k-1}(1+\alpha B(t))^{k-1}}{w A(t)+\left(\chi H_{t}\right)^{k} G(t)} \widehat{X}_{t},
$$

where disposable wealth $\widehat{X}_{t}$ is defined in (4).

Proof: The Bellman equation is now $0=\mathcal{L}_{1} J+\mathcal{L}_{2} J$, where

$$
\begin{aligned}
\mathcal{L}_{1} J=\sup _{c, q, N}\{- & c J_{x}-(q-\bar{q}) h \chi J_{x}+\frac{1}{1-\gamma} c^{b(1-\gamma)}(q-\bar{q})^{(1-b)(1-\gamma)}+\alpha(q-\bar{q}) J_{\bar{q}} \\
+ & \left.\Gamma \zeta(t) N J_{x}+\zeta(t) \frac{w^{\gamma}}{1-\gamma}(x-N)^{1-\gamma}\right\},
\end{aligned}
$$




$$
\mathcal{L}_{2} J=J_{t}+r x J_{x}+y J_{x}+\left(r+\mu_{H}\right) h J_{h}+\mu_{Y} y J_{y}-\bar{q} h \chi J_{x}+(\alpha-\varepsilon) \bar{q} J_{\bar{q}}-(\delta+\zeta(t)) J .
$$

The first-order condition for the insurance variable $N$ implies

$$
N=x-w \Gamma^{-1 / \gamma} J_{x}^{-1 / \gamma}
$$

and

$$
\Gamma \zeta(t) N J_{x}+\zeta(t) \frac{w^{\gamma}}{1-\gamma}(x-N)^{1-\gamma}=\Gamma \zeta(t) x J_{x}+\frac{\gamma}{1-\gamma} \zeta(t) w \Gamma^{1-\frac{1}{\gamma}} J_{x}^{1-\frac{1}{\gamma}} .
$$

Note that in the case ignoring mortality risk, this term is simply zero. As in the case without bequest and mortality risk, the first-order conditions for $c, q$ imply

$$
c=b \hat{b}\left(\chi h-\alpha \frac{J_{\bar{q}}}{J_{x}}\right)^{k} J_{x}^{-\frac{1}{\gamma}}, \quad q=\bar{q}+(1-b) \hat{b}\left(\chi h-\alpha \frac{J_{\bar{q}}}{J_{x}}\right)^{k-1} J_{x}^{\frac{\gamma-1}{\gamma}} .
$$

Hence, $\mathcal{L}_{1} J$ becomes

$$
\mathcal{L}_{1} J=\Gamma \zeta(t) x J_{x}+\frac{\gamma}{1-\gamma}\left[w \zeta(t) \Gamma^{1-\frac{1}{\gamma}}+\hat{b}\left(\chi h-\alpha \frac{J_{\bar{q}}}{J_{x}}\right)^{k}\right] J_{x}^{1-\frac{1}{\gamma}}
$$

With a conjecture of the form

$$
J(t, x, h, y, \bar{q})=\frac{1}{1-\gamma}\left(w A(t)+(\chi h)^{k} G(t)\right)^{\gamma}(x+y F(t)-\bar{q} h \chi B(t))^{1-\gamma},
$$

we need $A(T)=1$ and $G(T)=F(T)=B(T)=0$ to satisfy the terminal condition. With $\hat{x}=x+y F(t)-\bar{q} h \chi B(t)$, we can write the relevant derivatives of $J$ as

$$
\begin{gathered}
J_{t}=\hat{x}^{-\gamma}\left(w A+(\chi h)^{k} G\right)^{\gamma-1}\left[\hat{x} \frac{\gamma}{1-\gamma}\left(w A^{\prime}+(\chi h)^{k} G^{\prime}\right)+\left(y F^{\prime}-\bar{q} h \chi B^{\prime}\right)\left(w A+(\chi h)^{k} G\right)\right], \\
J_{x}=\hat{x}^{-\gamma}\left(w A+(\chi h)^{k} G\right)^{\gamma}, \quad J_{\bar{q}}=-\hat{x}^{-\gamma}\left(w A+(\chi h)^{k} G\right)^{\gamma} h \chi B, \quad J_{y}=\hat{x}^{-\gamma}\left(w A+(\chi h)^{k} G\right)^{\gamma} F, \\
J_{h}=\hat{x}^{-\gamma}\left(w A+(\chi h)^{k} G\right)^{\gamma-1}\left[\hat{x} \frac{k \gamma}{1-\gamma}(\chi h)^{k-1} G-\bar{q} \chi B\left(w A+(\chi h)^{k} G\right)\right],
\end{gathered}
$$

where we are suppressing $t$-dependence for notational simplicity. Note that $J_{\bar{q}} / J_{x}=$ $-h \chi B$.

Now we get

$$
\begin{aligned}
& \mathcal{L}_{1} J=\hat{x}^{-\gamma}\left(w A+(\chi h)^{k} G\right)^{\gamma-1}\left\{\hat{x}\left[\frac{\gamma}{1-\gamma}\left(w \zeta \Gamma^{1-\frac{1}{\gamma}}+\hat{b}(1+\alpha B)^{k}(\chi h)^{k}\right)+\zeta \Gamma\left(w A+(\chi h)^{k} G\right)\right]\right. \\
&\left.-\zeta \Gamma(y F-\bar{q} h \chi B)\left(w A+(\chi h)^{k} G\right)\right\},
\end{aligned}
$$




$$
\begin{aligned}
\mathcal{L}_{2} J=\hat{x}^{-\gamma}\left(w A+(\chi h)^{k} G\right)^{\gamma-1}\{ & \hat{x}\left[w\left(\frac{\gamma}{1-\gamma} A^{\prime}+r A-\frac{\delta+\zeta}{1-\gamma} A\right)\right. \\
& \left.+\frac{\gamma}{1-\gamma}(\chi h)^{k}\left(G^{\prime}-\frac{\gamma-1}{\gamma} r G+\left(r+\mu_{H}\right) k G-\frac{\delta+\zeta}{\gamma} G\right)\right] \\
+ & \left.\left(w A+(\chi h)^{k} G\right)\right) y\left[F^{\prime}-\left(r-\mu_{Y}\right) F+1\right] \\
& \left.-\left(w A+(\chi h)^{k} G\right) \bar{q} \chi h\left[B^{\prime}-\left(\varepsilon-\alpha-\mu_{H}\right) B+1\right]\right\} .
\end{aligned}
$$

If we substitute these terms into the Bellman equation $0=\mathcal{L}_{1} J+\mathcal{L}_{2} J$ and divide by $\hat{x}^{-\gamma}\left(w A+(\chi h)^{k} G\right)^{\gamma-1}$, we obtain the equation

$$
\begin{aligned}
0=\hat{x} & {\left[\frac{\gamma}{1-\gamma} w\left(\zeta \Gamma^{1-\frac{1}{\gamma}}+\frac{1-\gamma}{\gamma}(\Gamma \zeta+r) A+A^{\prime}-\frac{\delta+\zeta}{\gamma} A\right)\right.} \\
& \left.\quad+\frac{\gamma}{1-\gamma}(\chi h)^{k}\left(\hat{b}(1+\alpha B)^{k}+\frac{1-\gamma}{\gamma}(\Gamma \zeta+r) G+G^{\prime}+\left(r+\mu_{H}\right) k G-\frac{\delta+\zeta}{\gamma} G\right)\right] \\
& +\left(w A+(\chi h)^{k} G\right)\left\{y\left[F^{\prime}-\left(r+\Gamma \zeta-\mu_{Y}\right) F+1\right]-\bar{q} h\left[B^{\prime}-\left(\Gamma \zeta+\varepsilon-\alpha-\mu_{H}\right) B+1\right]\right\} .
\end{aligned}
$$

Since this equation has to hold for all values of $\hat{x}, h, y$, we conclude that the functions $A$, $G, F$, and $B$ must satisfy the ODEs

$$
\begin{gathered}
0=A^{\prime}(t)-r_{A}(t) A(t)+\zeta(t), \\
0=G^{\prime}(t)-r_{G}(t) G(t)+\hat{b}(1+\alpha B(t))^{k}, \\
0=F^{\prime}(t)-r_{F}(t) F(t)+1, \quad 0=B^{\prime}(t)-r_{B}(t) B(t)+1,
\end{gathered}
$$

with $r_{A}(t), r_{B}(t), r_{G}(t)$, and $r_{F}(t)$ stated in (66)-(67). With $A(T)=1$ and $G(T)=F(T)=$ $B(T)=0$, the solutions are given by $(62)-(65)$.

By applying the conjecture for $J$, the first-order condition (69) leads to the optimal consumption strategy in (68). The optimal insurance is characterized by

$$
N=x-w \Gamma^{-1 / \gamma} \frac{\hat{x}}{w A(t)+(\chi h)^{k} G(t)}
$$

Note that $N=x$ without bequest, as the agent optimally gives up all wealth upon death. 


\section{B.2 The model of Section 4.2}

For the complete markets model of Section 4.2, we generalize preferences to bequest and mortality risk as follows:

$J(t, x, h, y, \bar{q})=\sup _{c, q, N, \Pi_{S}, \Pi_{H}} \mathrm{E}_{t}\left[\int_{t}^{\tau} e^{-\delta(s-t)} U\left(c_{s}, q_{s}, \bar{q}_{s}\right) d s+e^{-\delta(\tau-t)} \frac{w^{\gamma}}{1-\gamma}\left(X_{\tau-}-N\right)^{1-\gamma}\right]$,

where $\tau \leq T$ is the random time of death with associated mortality intensity $\zeta(t)$, and $N$ is the amount of insurance. The terminal condition is $J(T, x, h, y, \bar{q})=w^{\gamma} \frac{1}{1-\gamma} x^{1-\gamma}$.

Theorem 5 The value function is

$$
J(t, x, h, y, \bar{q})=\frac{1}{1-\gamma}\left(w A(t)+(\chi h)^{k} G(t)\right)^{\gamma}(x+y F(t)-\bar{q} \chi h B(t))^{1-\gamma},
$$

where $A, G, F$, and $B$ are given as in (62)-(65) but with

$$
\begin{gathered}
r_{A}(t)=\frac{\delta+\zeta(t)}{\gamma}+\frac{\gamma-1}{\gamma}(r+\Gamma \zeta(t))+\frac{\gamma-1}{2 \gamma^{2}} \Lambda^{2}, \\
r_{G}(t)=r_{A}(t)-k\left(r+\frac{\mu_{H}}{\gamma}-\frac{\gamma-1}{\gamma}(\chi-m)+\frac{1}{2}(k-1) \sigma_{H}^{2}\right), \\
r_{B}(t)=\Gamma \zeta(t)+\varepsilon-\alpha+\chi-m, \\
r_{F}(t)=r+\Gamma \zeta(t)-\mu_{Y}(t)+\sigma_{Y}(t)\left(\lambda_{S} \rho_{Y S}+\tilde{\rho}_{H Y}\left[\lambda_{H}-\rho_{H S} \lambda_{S}\right]\right) .
\end{gathered}
$$

With disposable wealth $\widehat{X}_{t}$ defined in (4), the optimal consumption decisions are given by (68), and the optimal investment strategy is

$$
\begin{aligned}
\Pi_{S t} & =\frac{1}{\gamma} \frac{\lambda_{S}-\rho_{H S} \lambda_{H}}{\sigma_{S}\left(1-\rho_{H S}^{2}\right)} \frac{\widehat{X}_{t}}{X_{t}}-\frac{\sigma_{Y}(t)}{\sigma_{S}}\left(\rho_{Y S}-\rho_{H S} \tilde{\rho}_{H Y}\right) \frac{Y_{t} F(t)}{X_{t}} \\
\Pi_{H t} & =\frac{1}{\gamma} \frac{\lambda_{H}-\rho_{H S} \lambda_{S}}{\sigma_{H}\left(1-\rho_{H S}^{2}\right)} \frac{\widehat{X}_{t}}{X_{t}}-\frac{\sigma_{Y}(t)}{\sigma_{H}} \tilde{\rho}_{H Y} \frac{Y_{t} F(t)}{X_{t}}+\frac{k\left(\chi H_{t}\right)^{k} G(t)}{w A(t)+\left(\chi H_{t}\right)^{k} G(t)} \frac{\widehat{X}_{t}}{X_{t}}+\frac{\bar{q}_{t} B(t) \chi H_{t}}{X_{t}} .
\end{aligned}
$$

Note that the stock investment is unchanged and that only the third term in the housing investment is changed because of the bequest. The proof is available upon request, but follows the proof of Theorem 2 closely with changes similar to those outlined in the above proof of Theorem 4.

\section{B.3 The full-fledged model of Section 5}

Finally, we extend Theorem 3 to bequest and mortality risk: 
Theorem 6 For $\theta \in \Theta$, the value function in the artificial market $\mathcal{M}_{\theta}$ is

$$
J(t, x, h, y, \bar{q})=\frac{1}{1-\gamma}\left(w A(t)+(\chi h)^{k} G(t)\right)^{\gamma}(x+y F(t)-\bar{q} \chi h B(t))^{1-\gamma},
$$

where

$$
\begin{gathered}
A(t)=e^{-\int_{t}^{T} r_{A}(s) d s}+\int_{t}^{T} e^{-\int_{t}^{u} r_{A}(s) d s} \zeta(u) d u, \\
B(t)=\int_{t}^{T} e^{-\int_{t}^{s} r_{B}(u) d u} d s, \\
G(t)=\hat{b} \int_{t}^{T} e^{-\int_{t}^{s} r_{G}(u) d u}(1+\alpha B(s))^{k} d s, \\
F(t)= \begin{cases}\int_{t}^{T} e^{-\int_{t}^{u} r_{F}(s) d s} d u, & t \in[\tilde{T}, T], \\
\int_{t}^{\tilde{T}} e^{-\int_{t}^{u} r_{F}(s) d s} d u+\Upsilon \int_{\tilde{T}}^{T} e^{-\int_{t}^{u} r_{F}(s) d s} d u, & t<\tilde{T}, \\
r_{A}(t)=\frac{\delta+\zeta(t)}{\gamma}+\frac{\gamma-1}{\gamma}(\tilde{r}(t)+\Gamma \zeta(t))+\frac{\gamma-1}{2 \gamma^{2}} \tilde{\Lambda}(t)^{2}, \\
r_{B}(t)=r_{\omega}(t)+\Gamma \zeta(t)+\varepsilon-\alpha+\chi-m, \\
r_{G}(t)=r_{A}(t)-k\left(\tilde{r}(t)-r_{\omega}(t)+\frac{\tilde{\mu}_{H}(t)}{\gamma}-\frac{\gamma-1}{\gamma}(\chi-m)+\frac{k-1}{2} \sigma_{H}^{2}\right), \\
r_{F}(t)=\tilde{r}(t)+\Gamma \zeta(t)-\mu_{Y}(t)+\sigma_{Y}(t)\left(\psi_{S} \tilde{\lambda}_{S}(t)+\psi_{H} \tilde{\lambda}_{H}(t)+\psi_{Y} \nu_{I}(t)\right) .\end{cases}
\end{gathered}
$$

Defining disposable wealth as

$$
\widehat{X}_{t}=X_{t}+Y_{t} F_{\theta}(t)-\bar{q}_{t} \tilde{H}_{t} B_{\theta}(t)
$$

the optimal consumption and investment strategy is given by

$$
\begin{aligned}
c_{t} & =b \hat{b} \frac{\left(\chi H_{t}\right)^{k}(1+\alpha B(t))^{k}}{w A(t)+\left(\chi H_{t}\right)^{k} G(t)} \widehat{X}_{t}, \\
q_{t} & =\bar{q}_{t}+(1-b) \hat{b} \frac{\left(\chi H_{t}\right)^{k-1}(1+\alpha B(t))^{k-1}}{w A(t)+\left(\chi H_{t}\right)^{k} G(t)} \widehat{X}_{t}, \\
\Pi_{S t} & =\frac{\xi_{S}(t)}{\gamma \sigma_{S}} \frac{\widehat{X}_{t}}{X_{t}}-\frac{\sigma_{Y}(t) \psi_{S}}{\sigma_{S}} \frac{Y_{t} F_{\theta}(t)}{X_{t}}, \\
\Pi_{H t} & =\frac{\xi_{H}(t)}{\gamma \sigma_{H}} \frac{\widehat{X}_{t}}{X_{t}}-\frac{\sigma_{Y}(t) \psi_{H}}{\sigma_{H}} \frac{Y_{t} F_{\theta}(t)}{X_{t}}+k \frac{\left(\chi H_{t}\right)^{k} G(t)}{w A(t)+\left(\chi H_{t}\right)^{k} G(t)} \frac{\widehat{X}_{t}}{X_{t}}+\bar{q}_{t} \chi B_{\theta}(t) \frac{H_{t}}{X_{t}}, \\
\Pi_{I t} & =\frac{\xi_{I}(t)}{\gamma} \frac{\widehat{X}_{t}}{X_{t}}-\sigma_{Y}(t) \psi_{Y} \frac{Y_{t} F_{\theta}(t)}{X_{t}}
\end{aligned}
$$

with $\xi_{S}(t), \xi_{H}(t), \xi_{I}(t)$ are defined in terms of the Sharpe ratios and the correlation structure, cf. (57)-(59) in Appendix A.4. 
The proof is available upon request, but follows the proof of Theorem 3 closely with changes similar to those outlined in the above proof of Theorem 4. 


\section{References}

Abel, A. B. (1990). Asset Prices under Habit Formation and Catching up with the Joneses. American Economic Review 80(2), 38-42.

Andrews, D. and A. C. Sánchez (2011). The Evolution of Homeownership Rates in Selected OECD Countries: Demographic and Public Policy Influences. OECD Journal: Economic Studies 2011(1), 207-243.

Ang, A. (2014). Asset Management. Oxford University Press.

Attanasio, O. P., R. Bottazzi, H. W. Low, L. Nesheim, and M. Wakefield (2012). Modelling the Demand for Housing over the Life Cycle. Review of Economic Dynamics $15(1), 1-18$.

Aydilek, A. (2013). Habit Formation and Housing over the Life Cycle. Economic Modelling 33, 858-866.

Benzoni, L., P. Collin-Dufresne, and R. S. Goldstein (2007). Portfolio Choice over the Life-Cycle when the Stock and Labor Markets are Cointegrated. Journal of Finance 62(5), 2123-2167.

Bick, B., H. Kraft, and C. Munk (2013). Solving Constrained Consumption-Investment Problems by Simulation of Artificial Market Strategies. Management Science 59(2), $485-503$.

van Binsbergen, J. H. (2013, September). Good-Specifc Habit Formation and the Cross Section of Expected Returns. Available at SSRN: http://ssrn.com/abstract= 1101456. Journal of Finance, forthcoming.

Blanchard, O. J. (1985). Debt, Deficits, and Finite Horizons. Journal of Political Economy $93(2), 223-247$.

Bodie, Z., R. C. Merton, and W. F. Samuelson (1992). Labor Supply Flexibility and Portfolio Choice in a Life Cycle Model. Journal of Economic Dynamics and Control 16, 427-449.

Bricker, J., A. B. Kennickell, K. B. Moore, and J. Sabelhaus (2012). Changes in U.S. Family Finances from 2007 to 2010: Evidence from the Survey of Consumer Finances. Federal Reserve Bulletin 98(2), 1-80.

Browning, M. and M. D. Collado (2007). Habits and Heterogeneity in Demands: A Panel Data Analysis. Journal of Applied Econometrics 22(3), 625-640.

Campbell, J. Y. (2006). Household Finance. Journal of Finance 61(4), 1553-1604.

Campbell, J. Y. and J. F. Cocco (2003). Household Risk Management and Optimal Mortgage Choice. Quarterly Journal of Economics 118(4), 1449-1494. 
Campbell, J. Y. and J. H. Cochrane (1999). By Force of Habit: A ConsumptionBased Explanation of Aggregate Stock Market Behavior. Journal of Political Economy 107(2), 205-251.

Carrasco, R., J. M. Labeaga, and J. D. Lopez-Salido (2005). Consumption and Habits: Evidence from Panel Data. The Economic Journal 115(500), 144-165.

Carroll, C. D. (1997). Buffer-Stock Saving and the Life Cycle/Permanent Income Hypothesis. Quarterly Journal of Economics 112(1), 1-55.

Cauley, S. D., A. D. Pavlov, and E. S. Schwartz (2007). Home Ownership as a Constraint on Asset Allocation. Journal of Real Estate Finance and Economics 34(3), 283-311.

Chetty, R. and A. Szeidl (2007). Consumption Commitments and Risk Preferences. Quarterly Journal of Economics 122(2), 831-877.

Chetty, R. and A. Szeidl (2014, October). The Effect of Housing on Portfolio Choice. NBER Working paper 15998, available at http://www.rajchetty.com/.

Choi, H.-S., H. G. Hong, J. D. Kubik, and J. P. Thompson (2013, December). When Real Estate is the Only Game in Town. Available at SSRN: http://ssrn.com/ abstract $=2373179$.

Cocco, J. F. (2005). Portfolio Choice in the Presence of Housing. Review of Financial Studies 18(2), 535-567.

Cocco, J. F., F. J. Gomes, and P. J. Maenhout (2005). Consumption and Portfolio Choice over the Life Cycle. Review of Financial Studies 18(2), 491-533.

Corradin, S., J. L. Fillat, and C. Vergara-Alert (2014). Optimal Portfolio Choice with Predictability in House Prices and Transaction Costs. Review of Financial Studies 27(4), 823-880.

Cotter, J. and R. Roll (2014). A Comparative Anatomy of Residential REITs and Private Real Estate Markets: Returns, Risks and Distributional Characteristics. Real Estate Economics, forthcoming.

Cvitanić, J. and I. Karatzas (1992). Convex Duality in Constrained Portfolio Optimization. Annals of Applied Probability 2(4), 767-818.

De Nardi, M., E. French, and J. B. Jones (2010). Why do the Elderly Save? The Role of Medical Expenses. Journal of Political Economy 118(1), 39-75.

Feigenbaum, J. (2008). Can Mortality Risk Explain the Consumption Hump? Journal of Macroeconomics 30(3), 844-872.

Fernández-Villaverde, J. and D. Krueger (2007). Consumption over the Life Cycle: Facts from Consumer Expenditure Survey Data. Review of Economics and Statistics 89(3), 
$552-565$.

Fischer, M. and M. Stamos (2013). Optimal Life Cycle Portfolio Choice with Housing Market Cycles. Review of Financial Studies 26(9), 2311-2352.

Flavin, M. and S. Nakagawa (2008). A Model of Housing in the Presence of Adjustment Costs: A Structural Intepretation of Habit Persistence. American Economic Review 98(1), 474-495.

Flavin, M. and T. Yamashita (2002). Owner-Occupied Housing and the Composition of the Household Portfolio. American Economic Review 91(1), 345-362.

Goetzmann, W. (1993). The Single Family Home in the Investment Portfolio. Journal of Real Estate Finance and Economics 6(3), 201-222.

Gomes, F. and A. Michaelides (2003). Portfolio Choice with Internal Habit Formation: A Life-Cycle Model with Uninsurable Labor Income Risk. Review of Economic Dynamics 6(4), 729-766.

Gourinchas, P.-O. and J. A. Parker (2002). Consumption Over the Life Cycle. Econometrica 70(1), 47-89.

Guiso, L. and P. Sodini (2013). Household Finance: An Emerging Field. Volume 2, Part B of Handbook of the Economics of Finance, Chapter 21, pp. 1397-1532. Elsevier.

Hansen, G. D. and S. İmrohoroğlu (2008). Consumption over the Life Cycle: The Role of Annuities. Review of Economic Dynamics 11(3), 566-583.

Heaton, J. and D. Lucas (1997). Market Frictions, Savings Behavior, and Portfolio Choice. Macroeconomic Dynamics 1(1), 76-101.

van Hemert, O. (2010). Household Interest Rate Risk Management. Real Estate Economics 38(3), 467-505.

Heyerdahl-Larsen, C. (2014). Asset Prices and Real Exchange Rates with Deep Habits. Review of Financial Studies 27(11), 3280-3317.

Hoesli, M. and E. Oikarinen (2012). Are REITs Real Estate? Evidence from International Sector Level Data. Journal of International Money and Finance 31(7), $1823-1850$.

Ingersoll, Jr., J. E. (1992). Optimal Consumption and Portfolio Rules with Intertemporally Dependent Utility of Consumption. Journal of Economic Dynamics and Control 16(3-4), 681-712.

Karatzas, I., J. Lehoczky, S. Shreve, and G. Xu (1991). Martingale and Duality Methods for Utility Maximization in an Incomplete Market. SIAM Journal on Control and Optimization 29(3), 702-730. 
Koijen, R. S. J., T. E. Nijman, and B. J. M. Werker (2010). When Can Life-cycle Investors Benefit from Time-varying Bond Risk Premia? Review of Financial Studies 23(2), 741-780.

Kraft, H. and C. Munk (2011). Optimal Housing, Consumption, and Investment Decisions over the Life-Cycle. Management Science 57(6), 1025-1041.

Lee, M.-L., M.-T. Lee, and K. Chiang (2008). Real Estate Risk Exposure of Equity Real Estate Investment Trusts. Journal of Real Estate Finance 85 Economics 36(2), $165-181$.

Li, W. and R. Yao (2007). The Life-Cycle Effects of House Price Changes. Journal of Money, Credit and Banking 39(6), 1375-1409.

Lynch, A. W. and S. Tan (2011). Labor Income Dynamics at Business-cycle Frequencies: Implications for Portfolio Choice. Journal of Financial Economics 101(2), 333-359.

Menzly, L., T. Santos, and P. Veronesi (2004). Understanding Predictability. Journal of Political Economy 112(1), 1-46.

Merton, R. C. (1969). Lifetime Portfolio Selection Under Uncertainty: The ContinuousTime Case. Review of Economics and Statistics 51(3), 247-257.

Merton, R. C. (1971). Optimum Consumption and Portfolio Rules in a Continuous-Time Model. Journal of Economic Theory 3(4), 373-413.

Munk, C. (2008). Portfolio and Consumption Choice with Stochastic Investment Opportunities and Habit Formation in Preferences. Journal of Economic Dynamics and Control 32(11), 3560-3589.

Munk, C. and C. Sørensen (2010). Dynamic Asset Allocation with Stochastic Income and Interest Rates. Journal of Financial Economics 96(3), 433-462.

Nagatani, K. (1972). Life Cycle Saving: Theory and Fact. American Economic Review $62(3), 344-353$.

Pagliari, J. L., K. A. Scherer, and R. T. Monopoli (2005). Public Versus Private Real Estate Equities: A More Refined, Long-Term Comparison. Real Estate Economics 33(1), 147-187.

Piazzesi, M., M. Schneider, and S. Tuzel (2007). Housing, Consumption, and Asset Pricing. Journal of Financial Economics 83(3), 531-569.

Polkovnichenko, V. (2007). Life Cycle Portfolio Choice with Additive Habit Formation Preferences and Uninsurable Labor Income Risk. Review of Financial Studies 20(1), $83-124$. 
Ravina, E. (2007, November). Habit Formation and Keeping Up with the Joneses: Evidence from Micro Data. Available at SSRN: http://ssrn. com/abstract=928248.

Ravn, M., S. Schmitt-Grohé, and M. Uribe (2006). Deep Habits. Review of Economic Studies 73(1), 195-218.

Richard, S. F. (1975). Optimal Consumption, Portfolio and Life Insurance Rules for an Uncertain Lived Individual in a Continuous Time Model. Journal of Financial Economics 2(2), 187-203.

Ryder, Jr., H. E. and G. M. Heal (1973). Optimal Growth with Intertemporally Dependent Preferences. Review of Economic Studies 40(1), 1-31.

Samuelson, P. A. (1969). Lifetime Portfolio Selection by Dynamic Stochastic Programming. Review of Economics and Statistics 51(3), 239-246.

Sinai, T. and N. S. Souleles (2005). Owner-Occupied Housing as a Hedge Aganist Rent Risk. Quarterly Journal of Economics 120(2), 763-789.

Solnick, S. J. and D. Hemenway (2005). Are Positional Concerns Stronger in Some Domains than in Others? American Economic Review 95(2), 147-151.

Thurow, L. (1969). The Optimum Lifetime Distribution of Consumption Expenditures. American Economic Review 59(3), 324-330.

Wachter, J. A. and M. Yogo (2010). Why Do Household Portfolio Shares Rise in Wealth? Review of Financial Studies 23(11), 3929-3965.

Yang, F. (2009). Consumption over the Life Cycle: How Different is Housing? Review of Economic Dynamics 12(3), 423-443.

Yao, R. and H. H. Zhang (2005). Optimal Consumption and Portfolio Choices with Risky Housing and Borrowing Constraints. Review of Financial Studies 18(1), 197239. 


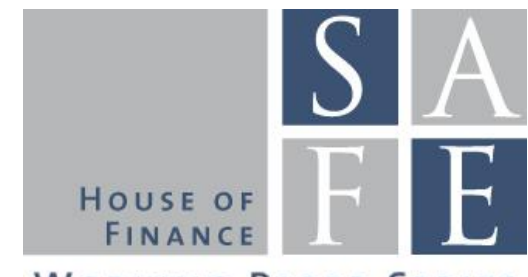

Working PAPER SERIES

\section{Recent Issues}

No. 84 Raimond Maurer, Olivia S. Mitchell, Ralph Rogalla, Tatjana Schimetschek

No. 83 Patrick Grüning

No. 82 Edgar Vogel, Alexander Ludwig, Axel Börsch-Supan

No. 81 Jens-Hinrich Binder

No. 80 Enrique G. Mendoza, Linda L. Tesar, Jing Zhang

No. 79 Òscar Jordà, Alan M. Taylor

No. 78 Harris Dellas, Dirk Niepelt

No. 77 Benjamin Born, Gernot J. Müller, Johannes Pfeifer

No. 76 Alberto Alesina, Carlo Favero, Francesco Giavazzi

No. 75 Markus Behn, Rainer Haselmann, Vikrant Vig

No. 74 Nicole Branger, Patrick Konermann, Christoph Meinerding, Christian Schlag

No. 73 Max Groneck, Alexander Ludwig, Alexander Zimpel

No. 72 Alexander Ludwig, Matthias Schön

No. 71 Daniel Harenberg, Alexander Ludwig
Will They Take the Money and Work? An

Empirical Analysis of People's Willingness to Delay Claiming Social Security Benefits for a Lump Sum

International Endogenous Growth, Macro Anomalies, and Asset Prices

Aging and Pension Reform: Extending the Retirement Age and Human Capital Formation

Resolution Planning and Structural Bank Reform within the Banking Union

Saving Europe?: The Unpleasant Arithmetic of Fiscal Austerity in Integrated Economies

The Time for Austerity: Estimating the Average Treatment Effect of Fiscal Policy

Austerity

Does Austerity Pay Off?

The Output Effect of Fiscal Consolidation Plans

The Limits of Model-Based Regulation

Equilibrium Asset Pricing in Networks with Mutually Exciting Jumps

A Life-Cycle Model with Ambiguous Survival Beliefs

Endogenous Grids in Higher Dimensions: Delaunay Interpolation and Hybrid Methods

Social Security in an Analytically Tractable Overlapping Generations Model with Aggregate and Idiosyncratic Risk 